\title{
EXACT CONTROLLABILITY OF A MULTILAYER RAO-NAKRA PLATE WITH CLAMPED BOUNDARY CONDITIONS
}

\author{
Scott W. Hansen ${ }^{1}$ and Oleg Imanuvilov ${ }^{2}$
}

\begin{abstract}
Exact controllability results for a multilayer plate system are obtained from the method of Carleman estimates. The multilayer plate system is a natural multilayer generalization of a classical three-layer "sandwich plate" system due to Rao and Nakra. The multilayer version involves a number of Lamé systems for plane elasticity coupled with a scalar Kirchhoff plate equation. The plate is assumed to be either clamped or hinged and controls are assumed to be locally distributed in a neighborhood of a portion of the boundary. The Carleman estimates developed for the coupled system are based on some new Carleman estimates for the Kirchhoff plate as well as some known Carleman estimates due to Imanuvilov and Yamamoto for the Lamé system.
\end{abstract}

Mathematics Subject Classification. 93B05, 93C20, 74K20.

Received November 7, 2008. Revised February 24, 2010 and May 3, 2010.

Published online November 8, 2010.

\section{INTRODUCTION}

The classical (three-layer) "sandwich plate" (or sandwich beam) is a model for a plate structure (or beam structure) consisting of two relatively stiff outer layers which "sandwich" a much more compliant central layer. Some of the better-known sandwich beam models to be found in the engineering literature include the models of Mead and Markus [19], DiTaranto [2], Rao and Nakra [20]; also see Sun and Lu [23] for history and comparisons of the models.

In Hansen [3] several multilayer generalizations of the classical sandwich plate models consisting of alternating "stiff" and "compliant" layers are derived. These models fall into two general types. In the case that all the kinetic energy (in-plane, transverse and rotational) is accounted for in the stiff layers, a generalization of the Rao-Nakra model is obtained. In the case that only transverse kinetic energy is accounted for, a simpler model (see also [4]) is obtained which can be viewed as a generalization of the Mead-Markus model.

In this article we prove exact controllability with locally distributed controls (in a region to be specified, sufficiently large) for the system (2.12), referred to as the "thin compliant layer Rao-Nakra model with shear

\footnotetext{
Keywords and phrases. Carleman estimates, exact controllability, multilayer plate, Lamé system, Kirchhoff plate.

1 Department of Mathematics, Iowa State University, Ames, IA 50011, USA.shansen@orion.math.iastate.edu

Supported in part by the National Science Foundation under grant DMS 02052148.

2 Department of Mathematics, Colorado State University, Ft. Collins, CO 80523, USA.

Supported in part by the National Science Foundation under grants DMS 02052148 and DMS 0808130.
} 
damping" in [3]. In the special case of only three plate layers the model under consideration is the following:

$$
\left.\begin{array}{rl}
m \partial_{x_{0}}^{2} w-\alpha \Delta_{x^{\prime}} \partial_{x_{0}}^{2} w+K \Delta_{x^{\prime}}^{2} w-\tilde{h} \operatorname{div}_{x^{\prime}}\left(G_{2} s^{2}+\tilde{G}_{2} \partial_{x_{0}} s^{2}\right) & =f_{3} \\
h_{1} \rho_{1} \partial_{x_{0}}^{2} v^{1}-12 h_{1} \hat{D}_{1} L^{1} v^{1}-G_{2} s^{2}-\tilde{G}_{2} \partial_{x_{0}} s^{2} & =\left\{f_{1}^{1}, f_{2}^{1}\right\} \\
h_{3} \rho_{3} \partial_{x_{0}}^{2} v^{3}-12 h_{3} \hat{D}_{3} L^{3} v^{3}+G_{2} s^{2}+\tilde{G}_{2} \partial_{x_{0}} s^{2} & =\left\{f_{1}^{3}, f_{2}^{3}\right\}
\end{array}\right\} \text { in } \Omega \times(0, \infty) .
$$

In the above, and throughout the paper we use $x_{0}$ to denote the time coordinate, while $x^{\prime}=\left\{x_{1}, x_{2}\right\}$ refers to the spatial coordinates. The variable $s^{2}=\left\{s_{1}^{2}, s_{2}^{2}\right\}$ represents the shear in the second layer and is defined in terms of the transverse displacement $w$ and in-plane displacements $v^{1}=\left\{v_{1}^{1}, v_{2}^{1}\right\}, v^{3}=\left\{v_{1}^{3}, v_{2}^{3}\right\}$ (of the first and third layer, respectively) by

$$
s^{2}=\frac{v^{3}-v^{1}}{h_{2}}+\frac{h_{1}+2 h_{2}+h_{3}}{2 h_{2}} \nabla_{x^{\prime}} w ; \quad \nabla_{x^{\prime}} w=\left(\partial_{x_{1}} w, \quad \partial_{x_{2}} w\right) .
$$

The symbols $L^{i}, i=1,3$ represent the stationary Lamé operators of plane elasticity for the $i$ th layer and other parameters appearing $\left(m, \alpha, K, h_{i}, G_{i}, \tilde{G}_{i}, \hat{D}_{i}, \tilde{h}=\left(h_{1}+2 h_{2}+h_{3}\right) / 2\right)$ are various (positive) physical constants (see Sect. 2).

Various boundary conditions may be specified on $\Gamma=\partial \Omega$. Here, we consider two types of boundary conditions: clamped and hinged. In the case of clamped boundary conditions, one has

$$
\partial_{\vec{n}} w=w=0, \quad v^{1}=v^{3}=0 \text { on } \Gamma \times(0, \infty),
$$

where $\partial_{\vec{n}}$ is the derivative operator in the outward normal direction along $\Gamma, \vec{n}$ is the outward unit normal. In the case of hinged boundary conditions, one has

$$
\partial_{\vec{n}}^{2} w=w=0, \quad v^{1}=v^{3}=0 \text { on } \Gamma \times(0, \infty)
$$

(where operator $\partial_{\vec{n}}^{2}$ denotes the second derivative in $\vec{n}$ direction).

We prove the exact controllability of the system (1.1) with clamped (1.2) or hinged boundary conditions (1.3) using controls $f_{3}, f^{1}=\left\{f_{1}^{1}, f_{2}^{1}\right\}$ and $f^{3}=\left\{f_{1}^{3}, f_{2}^{3}\right\}$ which are supported on a set $\omega \subset \Omega$ that may be arbitrarily small in measure, but satisfies certain geometric conditions related to the existence of an appropriate pseudoconvex function.

Actually, we prove this exact controllability result for the more general multilayer system (2.12)-(2.14). (See Thms. 3.1 and 3.2.) For simplicity, the model we consider is described for the case of constant coefficients, however, all our estimates used in this paper allow for smoothly varying (in time and space) coefficients. Precise statements of the main results are given in Sections 3 and 4.

Concerning previous controllability results for layered beam and plate systems, the moment method was used in $[5,22]$ to obtain exact controllability results for three-layer Mead-Markus beam and the multilayer Rao-Nakra beam, respectively. In [21] exact controllability of a three-layer Rao-Nakra beam was proved using the multiplier method. To our knowledge, the results of this paper are the first exact controllability result for a layered plate system.

On the other hand, there is an extensive literature on the topic of exact controllability of the classical (single-layer) plate systems, where classical multipliers are applied. As our system (1.1) resembles the Reissner plate system, we mention in particular the work of Lagnese [13], Lagnese and Lions [14], where boundary stabilization and exact boundary controllability results for the Reissner plate are obtained. For the Kirchhoff plate we mention $[12,14,15]$.

Our method is based upon application of Carleman estimates. The system (1.1) (and also the more general system (2.12)) consists of a Kirchhoff plate (the first equation in (1.1)) coupled with a number of (dynamic) Lamé systems for planar elasticity (the second and third equations in (1.1)). As the coupling between the subsystems is of low enough order as to not present a serious difficulty, the main technical issues we must 
overcome are (i) observability/Carleman estimates for Lamé systems, (ii) observability/Carleman estimates for the Kirchhoff plate.

While there is an extensive literature on application of Carleman estimates to controllability problems for scalar-valued partial differential equations (see e.g. [24]), much less is known for systems of partial differential equations coupled through principal terms, as is the case in Lamé systems. Carleman estimates for the Lamé systems are obtained with the use of recently derived Carleman estimates due to Imanuvilov and Yamamoto [9-11]. (See Lems. 4.2 and 4.3.)

Up to principal terms, the first equation in (1.1) is a Kirchhoff plate:

$$
m \partial_{x_{0}}^{2} w-\alpha \Delta_{x^{\prime}} \partial_{x_{0}}^{2} w+K \Delta_{x^{\prime}}^{2} w=f_{3} \quad \text { in } \Omega \times(0, \infty)
$$

where $w$ satisfies the boundary conditions in (1.2) or (1.3). This PDE has double characteristics and this prevents standard application of Carleman estimates due a loss of one order of the power of the large parameter ( $s$ in our paper). Much of the effort of this paper is directed overcoming this difficulty through a careful analysis of the factorization of the principal symbols associated with (1.4).

Our Carleman estimates for (1.4) are given in Lemmas 4.5 and 4.8. As a consequence we also obtain controllability results for a Kirchhoff plate which are stated in Corollary 3.1.

A difficulty of dealing with equation (1.4) might be easily observed from the following fact. The principal part of equation (1.4) can be obtained from the hyperbolic analog of the (nonlocal) Stokes system

$$
\partial_{x_{0}}^{2} y-K^{\prime} \Delta y+\nabla p=\tilde{f}, \quad \operatorname{div} y=0
$$

by introduction of the stream function $w ; y=\left(\partial_{x_{2}} w,-\partial_{x_{1}} w\right)$.

This paper is organized as follows. In Section 2 we describe in detail the multilayer sandwich plate system and provide some well-posedness results and a-priori estimates. In Section 3 we state the main controllability results. Section 4 contains the proof of the main results and associated observability estimates. An appendix is included to provide detailed proof to several technical results.

\section{Multilayer SANDWich Plate MOdel}

\subsection{Mathematical notations}

Throughout the paper we assume the following notations: $\Omega$ denotes a bounded domain in the plane with the boundary $\Gamma=\partial \Omega$ of regularity $C^{3} ; \partial_{\vec{n}}$ denotes the derivative in the direction of $\vec{n}$, the outward unit normal to $\Omega$ and $\partial_{\vec{n}}^{2}$ denotes the second normal derivative. Likewise $\partial_{\tan }$ is the derivative in the tangential direction along $\Gamma$.

We use the rectangular coordinates $x^{\prime}=\left\{x_{1}, x_{2}\right\}$ to denote points in $\Omega$ and $x_{0}$ denotes the time coordinate; $x=\left(x_{0}, x^{\prime}\right)$ generally denotes points in $(0, \infty) \times \Omega$. In addition, $\nabla=\left(\partial_{x_{0}}, \partial_{x_{1}}, \partial_{x_{2}}\right), \nabla_{x^{\prime}}=\left(\partial_{x_{1}}, \partial_{x_{2}}\right), \Delta_{x^{\prime}}=$ $\partial_{x_{1}}^{2}+\partial_{x_{2}}^{2}$, etc. If $A$ and $B$ are operators then $[A, B]=A B-B A$ stands for the commutator of these operators. We also denote $D=\left(D_{x_{0}}, D_{x_{1}}, D_{x_{2}}\right) ; D_{x_{k}}=\frac{1}{i} \partial_{x_{k}}(i=\sqrt{-1}, k=0,1,2)$.

\subsection{Model description}

The model under consideration is referred to as the "thin compliant layer Rao-Nakra model" in [3]. For the purpose of defining physical constants and expressions for energy, we give a summary of the derivation of the equations of motion. See [3] for a detailed derivation.

The multilayer sandwich plate is assumed to consist of $\mathcal{N}=2 \mathcal{M}+1$ layers of alternating "stiff" and "compliant" plate layers that occupy the three-dimensional region $\Omega \times(0, h)$ at equilibrium. The layers are indexed from 1 to $\mathcal{N}$, consecutively, with odd index $k \in \mathcal{O}_{\mathcal{N}}=\{1,3, \ldots, \mathcal{N}\}$ for stiff layers and even index $k \in \mathcal{E}_{\mathcal{N}}=\{2,4, \ldots, 2 \mathcal{M}\}$ for compliant layers. 
As is typical in plate theories, it is assumed that the transverse displacement is independent of $x_{3}$, the transverse independent variable. Thus we may use the scalar $w\left(x_{0}, x^{\prime}\right)$ to denote the transverse displacement at the point $x^{\prime} \in \Omega$. We let $v^{i}=\left\{v_{1}^{i}, v_{2}^{i}\right\} i=1,2, \ldots, \mathcal{N}$ denote the in-plane displacements along the mid-plane of the $i$ th layer.

It is assumed in that each layer has a uniform thickness and all the layers are bonded to one another so that no slip occurs. The Kirchhoff hypothesis applies to the stiff layers (i.e., normal sections remain normal during deformation) while the compliant layers allow shear. In either case the in-plane displacements are assumed to vary linearly as a function of the transverse coordinate, with no extension or contraction in the transverse direction. It follows that any displacement is completely determined by specification of the state variables $w$ and $v^{i}, i \in \mathcal{O}_{\mathcal{N}}$.

If $\theta$ and $\xi$ are $r \times k$ matrix-valued functions defined on $\bar{\Omega}$, by $\theta\left(x^{\prime}\right) \cdot \xi\left(x^{\prime}\right)$ we mean the scalar product in $\mathbb{R}^{r k}$. We also denote

$$
(\theta, \xi)_{L^{2}(\Omega)}=\int_{\Omega} \theta \cdot \xi \mathrm{d} x^{\prime}, \quad(\theta, \xi)_{L^{2}(\Gamma)}=\int_{\Gamma} \theta \cdot \xi \mathrm{d} \Gamma
$$

Let $\sigma_{j k}^{(i)}, \epsilon_{j k}^{(i)}$ denote the stress and strain tensors for the $i$ th layer. It is assumed that each layer is transversely isotropic, with the axis of isotropy normal to the surfaces. Let $E_{i}>0, \nu_{i}\left(0<\nu_{i}<1 / 2\right), G_{i}>0$ denote the Young's modulus, Poisson ratio and transverse shear modulus for the $i$ th layer. One can derive the twodimensional stress-strain relations as in e.g., Lagnese and Lions [14], assuming $\sigma_{33}^{(i)}$ to be negligible. Then one obtains for $i=1,2, \ldots, \mathcal{N}$ :

$$
\sigma_{11}^{(i)}=\frac{E_{i}}{1-\nu_{i}^{2}}\left(\epsilon_{11}^{(i)}+\nu_{i} \epsilon_{22}^{(i)}\right), \quad \sigma_{22}^{(i)}=\frac{E_{i}}{1-\nu_{i}^{2}}\left(\epsilon_{22}^{(i)}+\nu_{i} \epsilon_{11}^{(i)}\right), \quad \sigma_{12}^{(i)}=\frac{E_{i}}{1+\nu_{i}} \epsilon_{12}^{(i)} .
$$

As Kirchhoff assumptions apply to odd layers, for $i \in \mathcal{O}_{\mathcal{N}}$ we have $\epsilon_{12}^{(i)}=\epsilon_{13}^{(i)}=0$. Viscous damping due to shear is included in the compliant layers:

$$
\sigma_{k 3}^{(i)}=2 G_{i} \epsilon_{k 3}^{(i)}+\frac{\mathrm{d}}{\mathrm{d} x_{0}} 2 \tilde{G}_{i} \epsilon_{k 3}^{(i)}, \quad k=1,2 ; \quad i \in \mathcal{E}_{\mathcal{N}}
$$

where $\tilde{G}_{i}$ is the modulus of transverse shear viscosity.

Define the form $\ell^{\nu}(0<\nu<1 / 2)$ for functions $\theta\left(x^{\prime}\right)=\left\{\theta_{1}\left(x^{\prime}\right), \theta_{2}\left(x^{\prime}\right)\right\}$ by

$$
\begin{aligned}
\ell^{\nu}(\theta ; \hat{\theta})= & \left(\partial_{x_{1}} \theta_{1}, \partial_{x_{1}} \hat{\theta}_{1}\right)_{L^{2}(\Omega)}+\left(\partial_{x_{2}} \theta_{2}, \partial_{x_{2}} \hat{\theta}_{2}\right)_{L^{2}(\Omega)}+\left(\nu \partial_{x_{2}} \theta_{2}, \partial_{x_{1}} \hat{\theta}_{1}\right)_{L^{2}(\Omega)} \\
& +\left(\nu \partial_{x_{1}} \theta_{1}, \partial_{x_{2}} \hat{\theta}_{2}\right)_{L^{2}(\Omega)}+\left(\left(\frac{1-\nu}{2}\right)\left(\partial_{x_{2}} \theta_{1}+\partial_{x_{1}} \theta_{2}\right),\left(\partial_{x_{2}} \hat{\theta}_{1}+\partial_{x_{1}} \hat{\theta}_{2}\right)\right)_{L^{2}(\Omega)} .
\end{aligned}
$$

Using the previously described displacement assumptions (more precisely (2.1)-(2.4) of [3]), small strain assumptions for displacements ((2.6) of [3]) stress-strain relations (2.1), (2.2), and the Rao-Nakra modeling assumption that the in-plane inertia and bending stiffnesses of the even layers are small (negligible) in comparison to those of the surrounding odd layers ((3.9) of [3]), one obtains the following expressions for the resulting kinetic energy $\mathcal{E}_{K_{i}}$ and potential energy $\mathcal{E}_{P_{i}}$ for the $i$ th layer:

$$
\begin{aligned}
& \left(\mathcal{E}_{K}\right)_{i}=\left\{\begin{array}{lr}
\frac{1}{2} \int_{\Omega}\left\{\rho_{i} h_{i}\left|\partial_{x_{0}} w\right|^{2}+\alpha_{i}\left|\nabla_{x^{\prime}} \partial_{x_{0}} w\right|^{2}+\rho_{i} h_{i}\left|\partial_{x_{0}}\left(v^{i}\right)\right|^{2}\right\} \mathrm{d} x^{\prime} & i \in \mathcal{O}_{\mathcal{N}} \\
\frac{1}{2} \int_{\Omega} \rho_{i} h_{i}\left|\partial_{x_{0}} w\right|^{2} \mathrm{~d} x^{\prime} & i \in \mathcal{E}_{\mathcal{N}}
\end{array}\right. \\
& \left(\mathcal{E}_{P}\right)_{i}= \begin{cases}\frac{1}{2} \int_{\Omega}\left\{\ell^{\nu_{i}}\left(h_{i}^{3} \hat{D}_{i} \nabla_{x^{\prime}} w ; \nabla_{x^{\prime}} w\right)+12 \ell^{\nu_{i}}\left(h_{i} \hat{D}_{i} v^{i} ; v^{i}\right)\right\} \mathrm{d} x^{\prime} & i \in \mathcal{O}_{\mathcal{N}} \\
\frac{1}{2} \int_{\Omega} G_{i} h_{i}\left|s_{i}\right|^{2} \mathrm{~d} x^{\prime} & i \in \mathcal{E}_{\mathcal{N}} .\end{cases}
\end{aligned}
$$

In the above, $\hat{D}_{i}=E_{i} / 12\left(1-\nu_{i}^{2}\right), \alpha_{i}=\rho_{i} h_{i}^{3} / 12$ where $h_{i}>0$ the thickness (assumed constant), $\rho_{i}>0$ the volume density, all for the $i$ th layer. For simplicity we assume the layers are homogeneous, hence $\hat{D}_{i}, E_{i}, \nu_{i}$ are assumed constant in each layer. (The shear coefficients $G_{i}$ and $\tilde{G}_{i}$ and densities $\rho_{i}$ can vary spatially.) 
The variable $s_{i}$ is the shear of the $i$ th layer which is a linear combination of the state variables $\left\{v_{O}, w\right\}$, defined in (2.4) below.

Define the following matrices:

$$
\begin{array}{ll}
\mathbf{h}_{O}=\operatorname{diag}\left(h_{1}, h_{3}, \ldots, h_{\mathcal{N}}\right), & \mathbf{h}_{E}=\operatorname{diag}\left(h_{2}, h_{4}, \ldots, h_{\mathcal{N}-1}\right), \\
\mathbf{D}_{O}=\operatorname{diag}\left(\hat{D}_{1}, \hat{D}_{3}, \ldots, \hat{D}_{\mathcal{N}}\right), & \mathbf{p}_{O}=\operatorname{diag}\left(\rho_{1}, \rho_{3}, \ldots, \rho_{\mathcal{N}}\right), \\
\mathbf{G}_{E}=\operatorname{diag}\left(G_{2}, G_{4}, \ldots, G_{\mathcal{N}-1}\right), & \tilde{\mathbf{G}}_{E}=\operatorname{diag}\left(\tilde{G}_{2}, \tilde{G}_{4}, \ldots, \tilde{G}_{\mathcal{N}-1}\right) .
\end{array}
$$

Also define $\overrightarrow{1}_{E} \in \mathbb{R}^{\mathcal{M}}$ and $\overrightarrow{1}_{O} \in \mathbb{R}^{\mathcal{M}+1}$ as the column vectors consisting entirely of 1 's.

Let $v_{O}$ denote the $(\mathcal{M}+1) \times 2$ matrix with rows $v^{i}, i \in \mathcal{O}_{\mathcal{N}}$. Likewise, let $s_{O}$ and $s_{E}$ denote the matrices with rows $s^{i}, i$ odd and even, respectively. Since the Kirchhoff hypothesis applies to the odd layers, $s_{O}=0$. Using the displacement assumptions it is possible to solve for $s_{E}$ in terms of $v_{O}, \nabla_{x^{\prime}} w$ :

$$
\mathbf{h}_{E} s_{E}=B v_{O}+\mathbf{h}_{E} \vec{N} \nabla_{x^{\prime}} w ; \quad \vec{N}=\mathbf{h}_{E}^{-1} A \mathbf{h}_{O} \overrightarrow{1}_{O}+\overrightarrow{1}_{E}
$$

where $A=\left(a_{i j}\right), B=\left(b_{i j}\right)$ are the $\mathcal{M} \times(\mathcal{M}+1)$ matrices defined by

$$
a_{i j}=\left\{\begin{array}{ll}
1 / 2 & \text { if } j=i \text { or } j=i+1 \\
0 & \text { otherwise }
\end{array} \quad b_{i j}= \begin{cases}(-1)^{i+j+1} & \text { if } j=i \text { or } j=i+1 \\
0 & \text { otherwise }\end{cases}\right.
$$

Also define

$$
\ell_{O}\left(v_{O}, \hat{v}_{O}\right)=\sum_{i \in \mathcal{O}_{\mathcal{N}}} \ell^{\nu_{i}}\left(v^{i} ; \hat{v}^{i}\right)
$$

Collecting the energies one finds that the total potential and kinetic energy may be expressed as

$$
\mathcal{E}_{K}\left(x_{0}\right)=c\left(\partial_{x_{0}} v_{O}, \partial_{x_{0}} w ; \partial_{x_{0}} v_{O}, \partial_{x_{0}} w\right) / 2 \quad \mathcal{E}_{P}\left(x_{0}\right)=a\left(v_{O}, w ; v_{O}, w\right) / 2,
$$

where $c(\cdot ; \cdot)$ and $a(\cdot ; \cdot)$ denote the bilinear forms

$$
\begin{aligned}
c\left(v_{O}, w ; \hat{v}_{O}, \hat{w}\right) & =(m w, \hat{w})_{L^{2}(\Omega)}+\left(\left(\mathbf{p}_{O} \mathbf{h}_{O}^{3} / 12\right) \overrightarrow{1}_{O} \nabla_{x^{\prime}} w, \overrightarrow{1}_{O} \nabla_{x^{\prime}} \hat{w}\right)_{L^{2}(\Omega)}+\left(\mathbf{h}_{O} \mathbf{p}_{O} v_{O}, \hat{v}_{O}\right)_{L^{2}(\Omega)} \\
& =(m w, \hat{w})_{L^{2}(\Omega)}+\left(\alpha \nabla_{x^{\prime}} w, \nabla_{x^{\prime}} \hat{w}\right)_{L^{2}(\Omega)}+\left(\mathbf{h}_{O} \mathbf{p}_{O} v_{O}, \hat{v}_{O}\right)_{L^{2}(\Omega)}, \\
a\left(v_{O}, w ; \hat{v}_{O}, \hat{w}\right) & =\ell_{O}\left(\mathbf{h}_{O}^{3} \mathbf{D}_{O} \overrightarrow{1}_{O} \nabla_{x^{\prime}} w ; \overrightarrow{1}_{O} \nabla_{x^{\prime}} \hat{w}\right)+12 \ell_{O}\left(\mathbf{h}_{O} \mathbf{D}_{O} v_{O} ; \hat{v}_{O}\right)+\left(\mathbf{G}_{E} \mathbf{h}_{E} s_{E}, \hat{s}_{E}\right)_{L^{2}(\Omega)} \\
& =\ell^{\bar{\nu}}\left(K \nabla_{x^{\prime}} w ; \nabla_{x^{\prime}} \hat{w}\right)+12 \ell_{O}\left(\mathbf{h}_{O} \mathbf{D}_{O} v_{O} ; \hat{v}_{O}\right)+\left(\mathbf{G}_{E} \mathbf{h}_{E} s_{E}, \hat{s}_{E}\right)_{L^{2}(\Omega)},
\end{aligned}
$$

where $s_{E}$ and $\hat{s}_{E}$ satisfy (2.4), and

$$
m=\sum_{i=1}^{\mathcal{N}} h_{i} \rho_{i}, \quad \alpha=\frac{1}{12} \sum_{i \in \mathcal{O}_{\mathcal{N}}} \rho_{i} h_{i}^{3}, \quad K=\sum_{i \in \mathcal{O}_{\mathcal{N}}} \hat{D}_{i} h_{i}^{3}, \quad \bar{\nu}=\frac{1}{K} \sum_{i \in \mathcal{O}_{\mathcal{N}}} D_{i} h_{i}^{3} \nu_{i}
$$

Let us assume the plate is clamped on a portion $\Gamma_{0}$ of the boundary $\Gamma$ and simply-supported on the complementary portion $\Gamma_{1}$. We also consider the possibility of transverse and in-plane forces distributed on a subdomain $\omega$ of $\Omega$.

The conservative equations of motion are easily obtained from the energy using Hamilton's principle. The equations of motion with damping (2.2) can then be included through the correspondence:

$$
\mathbf{G}_{E} \rightarrow \mathcal{G}_{E}:=\mathbf{G}_{E}+\tilde{\mathbf{G}}_{E} \frac{\partial}{\partial x_{0}}
$$


One obtains the following variational differential equation:

$$
\begin{aligned}
\left(m \partial_{x_{0}}^{2} w, \hat{w}\right)_{L^{2}(\Omega)}+\left(\alpha \nabla_{x^{\prime}} \partial_{x_{0}}^{2} w, \nabla_{x^{\prime}} \hat{w}\right)_{L^{2}(\Omega)}+\left(\mathbf{h}_{O} \mathbf{p}_{O} \partial_{x_{0}}^{2} v_{O}, \hat{v}_{O}\right)_{L^{2}(\Omega)}+\ell^{\bar{\nu}}\left(K \nabla_{x^{\prime}} w, \nabla_{x^{\prime}} \hat{w}\right) \\
\quad+12 \ell_{O}\left(\mathbf{h}_{O} \mathbf{D}_{O} v_{O}, \hat{v}_{O}\right)+\left(\mathbf{h}_{E} \mathcal{G}_{E} s_{E}, \mathbf{h}_{E}^{-1} B \hat{v}_{O}+N \nabla_{x^{\prime}} \hat{w}\right)_{L^{2}(\Omega)}=W\left(\left\{\hat{v}_{O}, \hat{w}\right\}\right)=\int_{\omega}\left(\hat{w} f_{3}+\hat{v}_{O} \cdot f_{O}\right) \mathrm{d} x^{\prime}
\end{aligned}
$$

In the above, $f_{3}$ is the (scalar) transverse applied force in $\omega$ and $f_{O}$ is the net in-plane force acting on the odd layers in $\omega$. ( $f_{O}$ has rows $f^{i}=\left\{f_{1}^{i}, f_{2}^{i}\right\}, i=1,3,5, \ldots, 2 \mathcal{M}+1$.) See [3] for a detailed description of the forces. The test functions $\hat{w}, \hat{v}_{O}$ are assumed to be compactly supported with respect to time $\left(x_{0}\right)$, have dimensions matching $w$ and $v_{O}$ respectively, and satisfy the satisfy the clamped boundary conditions on $\Gamma_{0}\left(i . e ., \hat{v}_{O}, \hat{w}\right.$ and its outward normal derivative $\partial_{\vec{n}} \hat{w}$ vanish on $\Gamma_{0}$ ) and on $\Gamma_{1}, \hat{w}$ and $\hat{v}_{O}$ vanish.

\subsection{Existence, uniqueness of solutions}

Define the spaces

$$
\begin{aligned}
H_{\Gamma_{0}}^{1}(\Omega) & =\left\{w \in H^{1}(\Omega): w=0 \text { on } \Gamma_{0}\right\} \\
H_{\Gamma_{0}}^{2}(\Omega) & =\left\{w \in H_{0}^{1}(\Omega): \partial_{x_{i}} w \in H_{\Gamma_{0}}^{1}(\Omega), i=1,2\right\} \\
\mathbf{L}^{2}(\Omega) & =\left\{y=\left\{y_{1}, y_{2}\right\}: y_{i} \in L^{2}(\Omega), i=1,2\right\} \\
\mathbf{H}^{1}(\Omega) & =\left\{y \in \mathbf{L}^{2}(\Omega): \partial_{x_{i}} y \in \mathbf{L}^{2}(\Omega), i=1,2\right\} \\
\mathbf{H}_{0}^{1}(\Omega) & =\left\{y \in \mathbf{H}^{1}(\Omega): y=0 \text { on } \Gamma\right\} \\
\mathbf{L}_{O}^{2}(\Omega) & =\left\{v_{O}=\left(v_{j}^{i}\right), i=1,3,5, \ldots \mathcal{N}, j=1,2: v_{j}^{i} \in L^{2}(\Omega)\right\} \\
\mathbf{H}_{O}^{1}(\Omega) & =\left\{v_{O} \in \mathbf{L}_{O}^{2}(\Omega): v_{j}^{i} \in H^{1}(\Omega)\right\} \\
\mathbf{H}_{O, 0}^{1}(\Omega) & =\left\{v_{O} \in \mathbf{H}_{O}^{1}(\Omega): v_{O}=0 \text { on } \Gamma\right\} .
\end{aligned}
$$

When $\Gamma=\Gamma_{0}, H_{\Gamma_{0}}^{k}(\Omega)=H_{0}^{k}(\Omega), k=1,2$. In the case $\Omega$ is replaced with another set, appropriate adjustments to the above definitions will be assumed.

The energy space is $\mathcal{V} \times \mathcal{H}$, where

$$
\mathcal{V}=\mathbf{H}_{O, 0}^{1}(\Omega) \times H_{\Gamma_{0}}^{2}(\Omega), \quad \mathcal{H}=\mathbf{L}_{O}^{2}(\Omega) \times H_{0}^{1}(\Omega)
$$

Define

$$
b\left(v_{O}, w ; \hat{v}_{O}, \hat{w}\right)=\left(\tilde{\mathbf{G}} \mathbf{h}_{E} s_{E}, \hat{s}_{E}\right)_{L^{2}(\Omega)},
$$

where $s_{E}$ is defined in terms of $v_{O}$ and $w$ by (2.4) and $\hat{s}_{E}$ is defined in terms of $\hat{v}_{O}$ and $\hat{w}$ by the same equation.

The variational formulation of the initial boundary value problem corresponding to (2.6) with clamped boundary conditions on $\Gamma_{0}$ and simply-supported boundary conditions on $\Gamma_{1}$ is the following:

Find $y=\left\{v_{O}, w\right\}$ such that

$$
\begin{aligned}
& y \in C([0, T], \mathcal{V}) \cap C^{1}([0, T], \mathcal{H}) \\
& c\left(\partial_{x_{0}}^{2} y ; \hat{y}\right)+b\left(\partial_{x_{0}} y ; \hat{y}\right)+a(y ; \hat{y})=\mathcal{W}(\hat{y}) \quad \forall \hat{y}=\left\{\hat{v}_{O}, \hat{w}\right\} \in \mathcal{V} \\
& \quad(\text { in the sense of distributions on }(0, T)) \\
& y(0, \cdot)=y^{0} \text { given in } \mathcal{V}, \quad \partial_{x_{0}} y(0, \cdot)=y^{1} \text { given in } \mathcal{H} .
\end{aligned}
$$

For simplicity, we assume all coefficients appearing in (2.8) are time-independent, positive and continuous on $\bar{\Omega}$, with thicknesses $h_{i}$, stiffnesses $\hat{D}_{i}$ and Poisson ratios $\nu_{i}$ constant in each layer. The forces $f_{3}, f_{O}$ in the definition of $\mathcal{W}$ are assumed to be in the class $L^{2}(Q)$ and $L^{2}\left(0, T ;\left(H^{1}(\Omega)\right)^{*}\right)$, respectively, where $\left(H^{1}(\Omega)\right)^{*}$ denotes the dual space to $H^{1}(\Omega)$.

We also assume $\partial \Omega=\Gamma_{0} \cup \Gamma_{1}$, where $\Gamma_{0} \cap \Gamma_{1}=\emptyset$. 
Using [3], standard variational theory (e.g., [1]) and regularity results of Lasiecka and Triggiani for the Kirchhoff plate [16]), one can prove the following.

Proposition 2.1. Suppose that $y^{0} \in \mathcal{V}, y^{1} \in \mathcal{H}, f_{3} \in L^{2}\left(0, T ;\left(H^{1}(\Omega)\right)^{*}\right), f_{O} \in L^{2}\left(0, T ; \mathbf{L}_{O}^{2}(\Omega)\right)$. There exists a unique solution to (2.7)-(2.9). Furthermore, there exists $C=C(T)>0$ such that for each $x_{0} \in[0, T]$

$$
\left\|\left\{y\left(x_{0}, \cdot\right), \partial_{x_{0}} y\left(x_{0}, \cdot\right)\right\}\right\|_{\mathcal{V} \times \mathcal{H}} \leq C\left\{\left\|y^{0}\right\|_{\mathcal{V}}+\left\|y^{1}\right\|_{\mathcal{H}}+\left\|f_{3}\right\|_{L^{2}\left(0, T ;\left(H^{1}(\Omega)\right)^{*}\right)}+\left\|f_{O}\right\|_{L^{2}\left(0, T ; \mathbf{L}_{O}^{2}(\Omega)\right)}\right\} .
$$

\subsection{Boundary value problem}

Define the Lamé operator with parameter $\nu$ by $L^{\nu}(D) \phi=\left\{L_{1}^{\nu}(D) \phi, L_{2}^{\nu}(D) \phi\right\}$ by

$$
\begin{aligned}
& L_{1}^{\nu}(D) \phi=\partial_{x_{1}}\left[\partial_{x_{1}} \phi_{1}+\nu \partial_{x_{2}} \phi_{2}\right]+\partial_{x_{2}}\left[\left(\frac{1-\nu}{2}\right)\left(\partial_{x_{2}} \phi_{1}+\partial_{x_{1}} \phi_{2}\right)\right]=\frac{1-\nu}{2} \Delta_{x^{\prime}} \phi_{1}+\frac{1+\nu}{2} \partial_{x_{1}}\left(\operatorname{div}_{x^{\prime}} \phi\right), \\
& L_{2}^{\nu}(D) \phi=\partial_{x_{2}}\left[\partial_{x_{2}} \phi_{2}+\nu \partial_{x_{1}} \phi_{1}\right]+\partial_{x_{1}}\left[\left(\frac{1-\nu}{2}\right)\left(\partial_{x_{1}} \phi_{2}+\partial_{x_{2}} \phi_{1}\right)\right]=\frac{1-\nu}{2} \Delta_{x^{\prime}} \phi_{2}+\frac{1+\nu}{2} \partial_{x_{2}}\left(\operatorname{div}_{x^{\prime}} \phi\right) .
\end{aligned}
$$

Also define the boundary operators $\mathcal{B}^{\nu}(D) \phi=\left\{\mathcal{B}_{1}^{\nu}(D)\left(\phi_{1}, \phi_{2}\right), \mathcal{B}_{2}^{\nu}(D)\left(\phi_{1}, \phi_{2}\right)\right\}$ by

$$
\begin{aligned}
& \mathcal{B}_{1}^{\nu}(D)\left(\phi_{1}, \phi_{2}\right)=\left[\left(\partial_{x_{1}} \phi_{1} n_{1}+\nu \partial_{x_{2}} \phi_{2} n_{1}\right)+\left(\frac{1-\nu}{2}\right)\left(\partial_{x_{2}} \phi_{1}+\partial_{x_{1}} \phi_{2}\right) n_{2}\right], \\
& \mathcal{B}_{2}^{\nu}(D)\left(\phi_{1}, \phi_{2}\right)=\left[\left(\partial_{x_{2}} \phi_{2} n_{2}+\nu \partial_{x_{1}} \phi_{1} n_{2}\right)+\left(\frac{1-\nu}{2}\right)\left(\partial_{x_{1}} \phi_{2}+\partial_{x_{2}} \phi_{1}\right) n_{1}\right],
\end{aligned}
$$

where $\vec{n}=\left(n_{1}, n_{2}\right)$ is the outward unit normal vector to $\Gamma$.

The following Green's formula is valid for all sufficiently smooth $\hat{\phi}, \phi$ :

$$
\ell^{\nu}(\phi, \hat{\phi})=\left(\mathcal{B}^{\nu}(D) \phi, \hat{\phi}\right)_{L^{2}(\Gamma)}-\left(L^{\nu}(D) \phi, \hat{\phi}\right)_{L^{2}(\Omega)} .
$$

For the function $\xi=\left(\xi_{j}^{i}\right)(i=1,3,5, \ldots, \mathcal{N}, j=1,2)$ define the matrices $L_{O}(D) \xi$ and $\mathcal{B}_{O}(x, D) \xi$ by

$$
\left(L_{O}(D) \xi\right)_{i j}=\left(L_{j}^{\nu_{i}}(D) \xi^{i}\right), \quad\left(\mathcal{B}_{O}(D) \xi\right)_{i j}=\left(\mathcal{B}_{j}^{\nu_{i}}(D) \xi^{i}\right), \quad i=1,3,5 \ldots, \mathcal{N}, j=1,2 .
$$

Assume that $\hat{w}$ is a sufficiently smooth function that vanishes along with its gradient on $\Gamma_{0}$. The equations of motion can be found using the Green's formula (2.11) and integrations by parts of (2.6). One obtains the following:

$$
\left.\begin{array}{rr}
m \partial_{x_{0}}^{2} w-\alpha \Delta_{x^{\prime}} \partial_{x_{0}}^{2} w+K \Delta_{x^{\prime}}^{2} w-\operatorname{div}_{x^{\prime}} \vec{N}^{T} \mathbf{h}_{E} \mathcal{G}_{E} s_{E}=f_{3} \\
\mathbf{h}_{O} \mathbf{p}_{O} \partial_{x_{0}}^{2} v_{O}-12 \mathbf{h}_{O} \mathbf{D}_{O} L_{O}(D) v_{O}+B^{T} \mathcal{G}_{E} s_{E}=f_{O}
\end{array}\right\} \quad \begin{aligned}
& \text { on } \Omega \times(0, \infty), \\
& \text { where } s_{E}=\mathbf{h}_{E}^{-1} B v_{O}+N \nabla_{x^{\prime}} w, \quad \mathcal{G}_{E} s_{E}=\mathbf{G}_{E} s_{E}+\tilde{\mathbf{G}}_{E} \partial_{x_{0}} s_{E} \\
&\left.\partial_{\vec{n}}^{2} w=0\right\} \text { on } \Gamma_{1} \times(0, \infty), \\
&\left.\partial_{\vec{n}} w=0\right\} \text { on } \Gamma_{0} \times(0, \infty), \\
&\left.w=0, \quad v_{O}=0\right\} \text { on } \Gamma \times(0, \infty) .
\end{aligned}
$$

Appropriate initial conditions compatible with finite energy solutions are of the form

$$
\left\{v_{O}(0, \cdot), w(0, \cdot)\right\}=\left\{v_{O}^{0}, w^{0}\right\} \quad \text { given in } \mathcal{V}, \quad\left\{\partial_{x_{0}} v_{O}(0, \cdot), \partial_{x_{0}} w(0, \cdot)\right\}=\left\{v_{O}^{1}, w^{1}\right\} \quad \text { given in } \mathcal{H} .
$$

Since we will need to refer to the literature on two-dimensional Lamé systems, it will be convenient to express the operators $L^{\nu}$ and boundary operators $\mathcal{B}^{\nu}$ in terms of effective 2-dimensional Lamé coefficients $\lambda(E, \nu), \mu(E, \nu)$. The three-dimensional Young's modulus $E$ and Poisson's ratio $\nu$ are related to the effective two-dimensional Young's modulus $\tilde{E}$ and Poisson's ratio $\tilde{\nu}$ by

$$
E=\frac{\tilde{E}}{1-\tilde{\nu}^{2}}, \quad \nu=\frac{\tilde{\nu}}{1-\tilde{\nu}},
$$


and these are related to the effective 2-dimensional Lamé parameters $\lambda, \mu$ by

$$
\tilde{E}=\frac{\mu(2 \mu+3 \lambda)}{\lambda+\mu}, \quad \tilde{\nu}=\frac{\lambda}{2(\lambda+\mu)}
$$

Since $E>0$ and $0<\nu<1 / 2$, it follows from $(2.17)$ that $0<\tilde{\nu}<1 / 3$ and $\tilde{E}>0$.

Henceforth we let $\lambda_{i}=\lambda\left(E_{i}, \nu_{i}\right), \mu_{i}=\mu\left(E_{i}, \nu_{i}\right)$ denote the effective (2-dimensional) Lamé coefficients for the $i$ th layer. Using (2.17), (2.18), one finds

$$
12 D_{i} L^{\nu_{i}}(D) \phi=\frac{E_{i}}{1-\nu_{i}^{2}} L^{\nu_{i}}(D) \phi=\left(\lambda_{i}+2 \mu_{i}\right) L^{\nu_{i}}(D) \phi=\mu_{i} \Delta_{x^{\prime}} \phi+\left(\lambda_{i}+\mu_{i}\right) \nabla_{x^{\prime}} \operatorname{div}_{x^{\prime}} \phi
$$

In terms of two-dimensional Lamé parameters, the boundary operator $\mathcal{B}^{\nu}(D)$ can also be written as

$$
(\lambda+2 \mu) \mathcal{B}^{\nu}(D) \phi=\mathbb{B}_{\lambda, \mu}(D) \phi=\left(n_{1} \sigma_{11}+n_{2} \sigma_{21}, n_{1} \sigma_{12}+n_{2} \sigma_{22}\right),
$$

where $\vec{n}=\left(n_{1}, n_{2}\right)$ is the unit normal vector on $\Gamma$ and

$$
\sigma_{j k}=\lambda \delta_{j k} \operatorname{div}_{x^{\prime}} \phi+\mu\left(\partial_{x_{k}} \phi_{j}+\partial_{x_{j}} \phi_{k}\right) ; \quad\left(\delta_{i j}=1 \text { if } i=j \text { and } 0 \text { otherwise }\right) .
$$

Remark 2.1. The system (2.12) in the case $\mathcal{M}=1$ becomes the system (1.1) in the introduction. In this case the various matrix quantities involved are

$$
\begin{gathered}
A=\left(\begin{array}{cc}
\frac{1}{2} & \frac{1}{2}
\end{array}\right), \quad B=\left(\begin{array}{cc}
-1 & 1
\end{array}\right), \quad \mathbf{h}_{O}=\operatorname{diag}\left(h_{1}, h_{3}\right), \quad \mathbf{h}_{E}=h_{2}, \\
\overrightarrow{1}_{O}=\left(\begin{array}{c}
1 \\
1
\end{array}\right), \quad \overrightarrow{1}_{E}=1, \quad \vec{N}=\mathbf{h}_{E}^{-1} A \mathbf{h}_{O} \overrightarrow{1}_{O}+\overrightarrow{1}_{E}=\frac{h_{1}+2 h_{2}+h_{3}}{2 h_{2}}, \\
\mathbf{D}_{O}=\operatorname{diag}\left(\hat{D}_{1}, \hat{D}_{3}\right), \quad \mathbf{p}_{O}=\operatorname{diag}\left(\rho_{1}, \rho_{3}\right), \quad \mathbf{G}_{E}=G_{2}, \tilde{\mathbf{G}}_{E}=\tilde{G}_{2}, \\
m=\rho_{1} h_{1}+\rho_{2} h_{2}+\rho_{3} h_{3}, \quad \alpha=\left(\rho_{1} h_{1}^{3}+\rho_{3} h_{3}^{3}\right) / 12, \quad K=\hat{D}_{1} h_{1}^{3}+\hat{D}_{3} h_{3}^{3}, \quad G_{E} \mathbf{h}_{E}=G_{2} h_{2} .
\end{gathered}
$$

The state variables are $w$ and $v_{O}$, where $v_{O}=\left(\begin{array}{c}v^{1} \\ v^{3}\end{array}\right)=\left(\begin{array}{cc}v_{1}^{1} & v_{3}^{1} \\ v_{1}^{3} & v_{2}^{3}\end{array}\right)$. The operator $L_{O}$ is defined by $L_{O}(D) v_{O}=\left(\begin{array}{c}L^{\nu_{1}}(D) v^{1} \\ L^{\nu_{3}}(D) v^{3}\end{array}\right)=\left(\begin{array}{cc}L_{1}^{\nu_{1}}(D) v^{1} & L_{2}^{\nu_{1}}(D) v^{1} \\ L_{1}^{\nu_{3}}(D) v^{3} & L_{2}^{\nu_{3}}(D) v^{3}\end{array}\right)$, where $L^{\nu}$ is defined in $(2.10)$.

\section{Statement of main Results}

In this section we describe our main controllability results. For simplicity, we consider the cases of clamped and simply-supported boundary conditions separately. In the clamped case, $\Gamma_{1}=\emptyset$ and $\Gamma=\Gamma_{0}$ and in the simply supported case, $\Gamma_{0}=\emptyset$ and $\Gamma=\Gamma_{1}$. In either case, we assume the control is locally distributed on the subdomain $\omega \subset \Omega$.

\subsection{Controllability problem}

Denote $Q=(0, T) \times \Omega, Q_{\omega}=(0, T) \times \omega$ and $\Sigma=(0, T) \times \Gamma$.

The equations $(2.12),(2.13),(2.14)$ with $\left(f_{3}, f_{O}\right)$ replaced by $\left(f_{3}+q_{3}, \chi_{\omega} f_{O}+q_{O}\right)$ become

$$
\begin{gathered}
\mathcal{P}_{1}(D)\left(w, v_{O}\right):=m \partial_{x_{0}}^{2} w-\alpha \Delta_{x^{\prime}} \partial_{x_{0}}^{2} w+K \Delta_{x^{\prime}}^{2} w-\operatorname{div}_{x^{\prime}} \vec{N}^{T} \mathbf{h}_{E} \mathcal{G}_{E} s_{E}=f_{3}+q_{3} \text { in } Q, \\
\mathcal{P}_{2}(D)\left(w, v_{O}\right):=\mathbf{h}_{O} \mathbf{p}_{O} \partial_{x_{0}}^{2} v_{O}-12 \mathbf{h}_{O} \mathbf{D}_{O} L_{O}(D) v_{O}+B^{T} \mathcal{G}_{E} s_{E}=\chi_{\omega} f_{O}+q_{O} \text { in } Q, \\
\text { where } s_{E}=\mathbf{h}_{E}^{-1} B v_{O}+\vec{N} \nabla_{x^{\prime}} w, \quad \mathcal{G}_{E} s_{E}=\mathbf{G}_{E} s_{E}+\tilde{\mathbf{G}}_{E} \partial_{x_{0}} s_{E} .
\end{gathered}
$$


Clamped boundary conditions are given by

$$
v_{O}=0, \quad w=0, \quad \partial_{\vec{n}} w=0 \quad \text { on } \quad \Sigma .
$$

The initial conditions are

$$
v_{O}(0, \cdot)=\mathbf{v}_{0}, \partial_{x_{0}} v_{O}(0, \cdot)=\mathbf{v}_{1}, w(0, \cdot)=w_{0}, \partial_{x_{0}} w(0, \cdot)=w_{1} .
$$

Here $f_{3}$ and $f_{O}$ are the locally distributed boundary controls over the subdomain $\omega \subset \Omega$.

We consider the following controllability problem for the system (3.1)-(3.4): Find controls $\left(f_{O}, f_{3}\right)$ supported in $Q_{\omega}$ such that at time $T$ the solution $\left\{v_{O}, w\right\}$ to $(3.1)-(3.4)$ satisfies

$$
v_{O}(T, \cdot)=\partial_{x_{0}} v_{O}(T, \cdot)=w(T, \cdot)=\partial_{x_{0}} w(T, \cdot)=0
$$

In the case of simply supported boundary conditions, (3.3) is replaced by

$$
v_{O}=0, \quad w=0, \quad \partial_{\vec{n}}^{2} w=0 \quad \text { on } \Gamma_{0} \times(0, T) .
$$

\subsection{Exact controllability results}

In order to formulate our results we need to introduce some notation. Let

$$
p(x, \xi)=a_{00}(x) \xi_{0}^{2}-\sum_{i, j=1}^{2} a_{i j}(x) \xi_{i} \xi_{j}
$$

be a symbol for a second order hyperbolic differential operator with coefficients $a_{i j} \in C^{1}(\bar{Q})$. Also let $\{p, q\}$ denote the Poisson bracket for symbols $p, q$ :

$$
\{p, q\}=\sum_{k=0}^{2}\left(\partial_{\xi_{k}} p\right)\left(\partial_{x_{k}} q\right)-\left(\partial_{x_{k}} p\right)\left(\partial_{\xi_{k}} q\right)
$$

Definition 3.1. We say that the function $\psi \in C^{2}(\bar{Q})$ is pseudoconvex respect to the symbol $p(x, \xi)$ if for all $\xi \in \mathbb{R}^{3} \backslash\{0\}$ the following inequalities hold:

(1) $\{p,\{p, \psi\}\}(x, \xi)>0$ for all $x \in \overline{Q \backslash Q_{\omega}}$ such that $p(x, \xi)=\left(\frac{\partial p}{\partial \xi}(x, \xi), \nabla \psi(x)\right)_{\mathbb{R}^{3}}=0$,

(2) $\{p(x, \xi-i s \nabla \psi(x)), p(x, \xi+i s \nabla \psi(x))\} / 2 i s>0$ for all $x \in \overline{Q \backslash Q_{\omega}}$ such that

$$
p(x, \xi+i s \nabla \psi(x))=\left(\frac{\partial p}{\partial \xi}(x, \xi+i s \nabla \psi(x)), \nabla \psi(x)\right)_{\mathbb{R}^{3}}=0 .
$$

Assumption A. There exists a function $\psi(x)$ for which (i)-(iv) below hold.

(i) $\psi$ is pseudoconvex respect to the following symbols

$$
\rho_{i} \xi_{0}^{2}-6 \hat{D}_{i}\left(1-\nu_{i}\right)\left(\xi_{1}^{2}+\xi_{2}^{2}\right), \quad \rho_{i} \xi_{0}^{2}-12 \hat{D}_{i}\left(\xi_{1}^{2}+\xi_{2}^{2}\right) \quad\left(i \in \mathcal{O}_{\mathcal{N}}\right) ; \quad \xi_{0}^{2}-\frac{K}{\alpha}\left(\xi_{1}^{2}+\xi_{2}^{2}\right)
$$

and

$$
\nabla_{x^{\prime}} \psi(x) \neq 0 \quad \forall x \in \overline{Q \backslash Q_{\omega}}
$$


(ii) For all $x \in \overline{(0, T) \times(\Gamma \backslash \partial \omega)}$ the following hold:

$$
\begin{aligned}
\partial_{\vec{n}} \psi & <0 \\
\sqrt{\rho_{i}}\left|\partial_{x_{0}} \psi\right| & <\frac{\mu_{i}}{\sqrt{\lambda_{i}+2 \mu_{i}}}\left|\partial_{\tan } \psi\right|+\frac{\sqrt{\mu_{i}} \sqrt{\lambda_{i}+\mu_{i}}}{\sqrt{\lambda_{i}+2 \mu_{i}}}\left|\partial_{\vec{n}} \psi\right| \quad i \in \mathcal{O}_{\mathcal{N}}, \\
\rho_{i}\left(\partial_{x_{0}} \psi\right)^{2}-\mu_{i}\left|\nabla_{x^{\prime}} \psi\right|^{2} & <0 \forall x \in \overline{(0, T) \times(\Gamma \backslash \partial \omega)} \quad i \in \mathcal{O}_{\mathcal{N}},
\end{aligned}
$$

where $\mu_{i}=6 \hat{D}_{i}\left(1-\nu_{i}\right), \lambda_{i}+2 \mu_{i}=12 \hat{D}_{i}$ and $\partial_{\tan }$ is the tangential derivative on $\Gamma$.

(iii) There exists a moment $\tau \in(0, T)$ such that

$$
\inf _{x^{\prime} \in \Omega \backslash \omega} \psi\left(\tau, x^{\prime}\right)>\max \left\{\sup _{x^{\prime} \in \Omega \backslash \omega} \psi\left(T, x^{\prime}\right), \sup _{x^{\prime} \in \Omega \backslash \omega} \psi\left(0, x^{\prime}\right)\right\} .
$$

(iv)

$$
\partial_{x_{0}} \psi\left(0, x^{\prime}\right)>0 \quad \partial_{x_{0}} \psi\left(T, x^{\prime}\right)<0 \quad \forall x^{\prime} \in \bar{\Omega} .
$$

Lemma 3.1. Let $\tilde{x} \notin \bar{\Omega}$, the subdomain $\omega$ taken such that $\left\{x^{\prime} \in \partial \Omega:\left(\vec{n}, x^{\prime}-\tilde{x}\right)_{\mathbb{R}^{2}} \leq 0\right\} \subset \partial \omega$. Let $\gamma \in(0, c)$ where

$$
c=\min _{i \in \mathcal{O}_{\mathcal{N}}}\left\{\frac{K}{\alpha}, \frac{12 \hat{D}_{i}}{\rho_{i}}, \frac{6 \hat{D}_{i}\left(1-\nu_{i}\right)}{\rho_{i}}\right\} .
$$

Then function $\psi(x)=-\gamma\left(x_{0}-\frac{T}{2}\right)^{2}+\left|x^{\prime}-\tilde{x}\right|^{2}$ satisfies Assumption A provided that $T$ is sufficiently large and $\gamma$ is sufficiently small.

Proof. In [7] it is is shown that the function $\psi(x)$ is pseudoconvex respect to the symbols

$$
\rho_{i} \xi_{0}^{2}-6 \hat{D}_{i}\left(1-\nu_{i}\right)\left(\xi_{1}^{2}+\xi_{2}^{2}\right), \quad \rho_{i} \xi_{0}^{2}-12 \hat{D}_{i}\left(\xi_{1}^{2}+\xi_{2}^{2}\right) \quad\left(i \in \mathcal{O}_{\mathcal{N}}\right) ; \quad \xi_{0}^{2}-\frac{K}{\alpha}\left(\xi_{1}^{2}+\xi_{2}^{2}\right)
$$

Since $\tilde{x}$ is taken outside of $\Omega$ the gradient of $\psi$ is not equal zero on $Q$. From the choice of $\psi$ the inequalities (3.13) follows immediately. And (3.9) follows from the fact that $\left\{x^{\prime} \in \partial \Omega:\left(\vec{n}, x^{\prime}-\tilde{x}\right)_{\mathbb{R}^{2}} \leq 0\right\} \subset \partial \omega$.

In order to have (3.12) we take $T$ such that

$$
\gamma \frac{T^{2}}{4}>d_{1}-d_{0}, \quad d_{0}=\min _{x^{\prime} \in \overline{\Omega \backslash \omega}}\left|x^{\prime}-\tilde{x}\right|^{2}, d_{1}=\max _{x^{\prime} \in \overline{\Omega \backslash \omega}}\left|x^{\prime}-\tilde{x}\right|^{2} .
$$

Moreover taking $\gamma=\epsilon, T=\epsilon^{-\frac{3}{4}}$ we still have the previous inequality satisfied for all sufficiently small positive $\epsilon$. On the other hand

$$
\sup _{x \in Q}\left|\partial_{x_{0}} \psi(x)\right| \leq 4 \epsilon^{\frac{1}{4}}
$$

and hence (3.10) and (3.11) are valid for $\epsilon$ sufficiently small. This completes the proof.

Our main controllability result for case of clamped boundary conditions is the following:

Theorem 3.1. Let $\mathbf{v}_{0} \in \mathbf{H}_{O, 0}^{1}(\Omega), \mathbf{v}_{1} \in \mathbf{L}_{O}^{2}(\Omega), w_{0} \in H_{0}^{2}(\Omega), w_{1} \in H_{0}^{1}(\Omega), q_{O} \in L^{2}(Q), q_{3} \in L^{2}\left(0, T ;\left(H^{1}(\Omega)\right)^{*}\right)$. Suppose that there exists a function $\psi$ which satisfies Assumption A. Then there exist controls $f_{O} \in \mathbf{L}_{O}^{2}\left(Q_{\omega}\right)$, $f_{3} \in\left(H^{1}(Q)\right)^{*}$; supp $f_{3} \subset Q_{\omega}$, such that there exists a solution $\left\{v_{O}, w\right\}$ to the problem (3.1) $-(3.5)$ with

$$
\begin{aligned}
v_{O} & \in L^{2}\left(0, T ; \mathbf{H}_{O, 0}^{1}(\Omega)\right), \partial_{x_{0}} v_{O} \in L^{2}\left(0, T ; \mathbf{L}_{O}^{2}(\Omega)\right), \\
w & \in L^{2}\left(0, T ; H_{0}^{2}(\Omega)\right), \partial_{x_{0}} w \in L^{2}\left(0, T ; H_{0}^{1}(\Omega)\right) .
\end{aligned}
$$

For the case of simply supported boundary conditions our main controllability result is the following: 
Theorem 3.2. Let $\mathbf{v}_{0} \in \mathbf{H}_{0}^{1}(\Omega), \mathbf{v}_{1} \in \mathbf{L}^{2}(\Omega), w_{0} \in H^{2}(\Omega) \cap H_{0}^{1}(\Omega), w_{1} \in H_{0}^{1}(\Omega)$ and assume Assumption $\mathrm{A}$ holds. Then there exist controls $f_{O} \in \mathbf{L}_{O}^{2}\left(Q_{\omega}\right), f_{3} \in L^{2}\left(Q_{\omega}\right)$, such that there exists a solution $\left\{v_{O}, w\right\}$ to the problem (3.1), (3.2), (3.6), (3.4), (3.5) with $v_{O} \in C^{0}\left([0, T] ; \mathbf{H}_{O}^{1}(\Omega)\right) \cap C^{1}\left([0, T] ; \mathbf{L}_{O}^{2}(\Omega)\right), w \in C^{0}\left([0, T] ; H^{2}(\Omega) \cap\right.$ $\left.H_{0}^{1}(\Omega)\right) \cap C^{1}\left([0, T] ; H_{0}^{1}(\Omega)\right)$.

Both theorems follow from Hilbert's Uniqueness method (Lions [18]) using observability estimates that we derive in the next section.

As a consequence of Theorems 3.1 and 3.2, analogous controllability results can be stated for the Lamé systems and the Kirchhoff plate. For the case of the Kirchhoff plate equation (1.4), with the initial conditions

$$
w(\cdot, 0)=w_{0}, \quad \partial_{x_{0}} w(\cdot, 0)=w_{1},
$$

we have the following controllability results.

Corollary 3.1. Assume that $T$ and $\omega \subset \Omega$ satisfy Assumption A. (That is, there exists a function $\psi(x)$ which is pseudoconvex with respect to the symbol $\xi_{0}^{2}-\frac{K}{\alpha}\left(\xi_{1}^{2}+\xi_{2}^{2}\right)$ which satisfies conditions (3.8), (3.9), (3.12), (3.13) of Assumption A.)

(i) Given any $w_{0} \in H_{0}^{2}(\Omega), w_{1} \in H_{0}^{1}(\Omega)$, there exists a control $f_{3} \in\left(H^{1}(Q)\right)^{*}$; $\operatorname{supp} f_{3} \subset Q_{\omega}$ such that the solution to (1.4), with clamped boundary conditions (1.2) and initial conditions (3.14) satisfies

$$
w \in L^{2}\left(0, \infty ; H_{0}^{2}(\Omega)\right), \partial_{x_{0}} w \in L^{2}\left(0, \infty ; H_{0}^{1}(\Omega)\right) \text { and } w \equiv 0 \quad \forall x_{0}>T .
$$

(ii) Given any $w_{0} \in H^{2}(\Omega) \cap H_{0}^{1}(\Omega)$, $w_{1} \in H_{0}^{1}(\Omega)$, there exist a control $f_{3} \in L^{2}\left(Q_{\omega}\right)$, such that the solution $w$ to the problem (1.4) with hinged boundary conditions (1.3) and initial conditions (3.14) satisfies

$$
w \in C^{0}\left([0, T] ; H^{2}(\Omega) \cap H_{0}^{1}(\Omega)\right) \cap C^{1}\left([0, T] ; H_{0}^{1}(\Omega)\right) \text { and } w(\cdot, T)=\partial_{x_{0}} w(\cdot, T)=0 .
$$

\section{Proof of MAin Results}

Before starting the proof we mention some known results on Carleman estimates for the hyperbolic equations and the Lamé system.

\subsection{Some known Carleman estimates}

Let $Q(D)=\partial_{x_{0}}^{2}-\frac{K}{\alpha} \Delta_{x^{\prime}}$ denote the wave operator and consider the following wave equation

$$
\begin{array}{r}
Q(D) y=f \text { in } Q, \quad y=g \text { on } \Sigma, \\
y(0, \cdot)=\partial_{x_{0}} y(0, \cdot)=\partial_{x_{0}} y(T, \cdot)=y(T, \cdot)=0 .
\end{array}
$$

For a given pseudoconvex function $\psi(x)$ it will be convenient to introduce the function

$$
\varphi(x)=\mathrm{e}^{\tau \psi(x)},
$$

where $\tau$ is a large positive parameter.

Lemma $4.1([7,25])$. Let $f_{0}, f_{1}, f_{2} \in L^{2}(Q), g \in L^{2}(\Sigma)$ and $\tilde{f} \in H^{-1}(Q)$. Let $\psi(x) \in C^{2}(\bar{Q})$ be a function pseudoconvex respect to the symbol $\xi_{0}^{2}-\frac{K}{\alpha}\left|\xi^{\prime}\right|^{2}$ such that $\left.\partial_{\vec{n}} \psi\right|_{(0, T) \times(\Gamma \backslash \partial \omega)}<0$ and the function $\varphi$ given by formula (4.2). Let $y \in L^{2}(Q)$ be a solution to problem (4.1) with the right hand side $f=\tilde{f}+\sum_{j=0}^{2} \partial_{x_{i}} f_{i}$. Then there exists $\tau_{0}>0$ such that for $\tau>\tau_{0}$ there exists $s_{0}(\tau)>0$ such that

$$
\int_{Q} s y^{2} \mathrm{e}^{2 s \varphi} \mathrm{d} x \leq C_{1}\left(\left\|\tilde{f} \mathrm{e}^{s \varphi}\right\|_{H^{-1}(Q)}^{2}+\sum_{i=0}^{2}\left\|f_{i} \mathrm{e}^{s \varphi}\right\|_{L^{2}(Q)}^{2}+\int_{Q_{\omega}} s y^{2} \mathrm{e}^{2 s \varphi} \mathrm{d} x+s\left\|g \mathrm{e}^{s \varphi}\right\|_{L^{2}(\Sigma)}^{2}\right) \quad \forall s \geq s_{0}(\tau)
$$


where $C_{1}$ is independent of $s$. Furthermore, if $f \in L^{2}(Q), g \in H^{1}(\Sigma)$, then

$$
\begin{aligned}
\int_{Q}\left(s \tau^{2} \mathrm{e}^{\tau \psi}|\nabla y|^{2}+s \tau^{4} \mathrm{e}^{3 \tau \psi} y^{2}\right) \mathrm{e}^{2 s \varphi} \mathrm{d} x \leq C_{1}\left(\left\|f \mathrm{e}^{s \varphi}\right\|_{L^{2}(Q)}^{2}+\int_{Q_{\omega}}\left(s \tau^{2} \mathrm{e}^{\tau \psi}|\nabla y|^{2}+s^{3} \tau^{4} \mathrm{e}^{3 \tau \psi} y^{2}\right) \mathrm{e}^{2 s \varphi} \mathrm{d} x\right. \\
\left.+\int_{\Sigma}\left(s \tau\left(\mathrm{e}^{\tau \psi}\left|\partial_{x_{0}} g\right|^{2}+\mathrm{e}^{\tau \psi}\left|\partial_{\tan } g\right|^{2}\right)+s^{3} \tau^{3} \mathrm{e}^{\tau \psi} g^{2}\right) \mathrm{e}^{s \varphi} \mathrm{d} \Sigma\right) \quad \forall \tau \geq \tau_{0}, s \geq s_{0}(\tau),
\end{aligned}
$$

where $\partial_{\tan }$ is the tangential derivative on $\Gamma$.

Next we consider the two-dimensional Lamé system

$$
\begin{array}{r}
P(x, D) \mathbf{u}=\rho\left(x^{\prime}\right) \partial_{x_{0}}^{2} \mathbf{u}-\mu\left(x^{\prime}\right) \Delta_{x^{\prime}} \mathbf{u}-\left(\mu\left(x^{\prime}\right)+\lambda\left(x^{\prime}\right)\right) \nabla_{x^{\prime}} \operatorname{div}_{x^{\prime}} \mathbf{u} \\
-\operatorname{div}_{x^{\prime}} \mathbf{u} \nabla_{x^{\prime}} \lambda\left(x^{\prime}\right)-\left(\nabla_{x^{\prime}} \mathbf{u}+\left(\nabla_{x^{\prime}} \mathbf{u}\right)^{T}\right) \nabla_{x^{\prime}} \mu\left(x^{\prime}\right)=\mathbf{f} \quad \text { in } Q=[0, T] \times \Omega, \\
\left.\mathbf{u}\right|_{\Sigma}=0, \quad \mathbf{u}\left(T, x^{\prime}\right)=\partial_{x_{0}} \mathbf{u}\left(T, x^{\prime}\right)=\mathbf{u}\left(0, x^{\prime}\right)=\partial_{x_{0}} \mathbf{u}\left(0, x^{\prime}\right)=0 \text { in } \Omega,
\end{array}
$$

where $\mathbf{u}=\left\{u_{1}, u_{2}\right\}, \mathbf{f}=\left\{f_{1}, f_{2}\right\}$ are vector functions and $\Gamma \in C^{3}$.

Remark 4.1. With $\rho, \lambda$ and $\mu$ picked to be $\rho_{i}, \lambda_{i}$, and $\mu_{i}$ (respectively) the operator $P(x, D)$ in $(4.5)$ is independent of $x$ and reduces to $P(D) \mathbf{u}=\rho_{i} \partial_{x_{0}}^{2} \mathbf{u}-12 D_{i} L^{\nu_{i}}(D) \mathbf{u}$, where $L^{\nu}(D)$ is the Lamé operator defined in (2.10). Here however, we only shall need to require that $\rho\left(x^{\prime}\right), \mu\left(x^{\prime}\right), \lambda\left(x^{\prime}\right) \in C^{2}(\bar{\Omega})$ and satisfy

$$
\rho\left(x^{\prime}\right)>0, \quad \mu\left(x^{\prime}\right)>0, \quad \mu\left(x^{\prime}\right)+\lambda\left(x^{\prime}\right)>0 \quad \text { in } \bar{\Omega} .
$$

Assumption $\mathbf{A}^{\prime}$. There exists a function $\psi(x)$ for which:

(i) $\psi$ is pseudoconvex respect to the following symbols

$$
\rho \xi_{0}^{2}-\mu\left(\xi_{1}^{2}+\xi_{2}^{2}\right), \quad \rho \xi_{0}^{2}-(\lambda+2 \mu)\left(\xi_{1}^{2}+\xi_{2}^{2}\right) ;
$$

(ii) for all $x \in \overline{(0, T) \times(\Gamma \backslash \partial \omega)}$ the following hold:

$$
\begin{aligned}
\partial_{\vec{n}} \psi & <0, \\
\sqrt{\rho}\left|\partial_{x_{0}} \psi\right| & <\frac{\mu}{\sqrt{\lambda+2 \mu}}\left|\partial_{\tan } \psi\right|+\frac{\sqrt{\mu} \sqrt{\lambda+\mu}}{\sqrt{\lambda+2 \mu}}\left|\partial_{\vec{n}} \psi\right|, \\
\rho\left(\partial_{x_{0}} \psi\right)^{2}-\mu\left|\nabla_{x^{\prime}} \psi\right|^{2} & <0 \quad \forall x \in \overline{(0, T) \times(\Gamma \backslash \partial \omega) .}
\end{aligned}
$$

Lemma $4.2([9])$. Let $\mathbf{f} \in \mathbf{L}^{2}(Q)$ and let the function $\varphi$ satisfy (4.2) where $\psi$ satisfies Assumption $\mathrm{A}^{\prime}$ and assume the Lamé coefficients satisfy (4.7). Then there exists $\widehat{\tau}>0$ such that for any $\tau>\widehat{\tau}$, there exists $s_{0}=s_{0}(\tau)>0$ such that for any solution $\mathbf{u} \in \mathbf{H}^{1}(Q)$ to problem (4.5)-(4.6), the following estimate holds for all $s \geq s_{0}(\tau):$

$$
\int_{Q}\left(|\nabla \mathbf{u}|^{2}+s^{2}|\mathbf{u}|^{2}\right) \mathrm{e}^{2 s \varphi} \mathrm{d} x \leq C_{2}\left(\left\|\mathbf{f e}^{s \varphi}\right\|_{\mathbf{L}^{2}(Q)}^{2}+\int_{Q_{\omega}}\left(|\nabla \mathbf{u}|^{2}+s^{2}|\mathbf{u}|^{2}\right) \mathrm{e}^{2 s \varphi} \mathrm{d} x\right)
$$

where the constant $C_{2}=C_{2}(\tau)>0$ is independent of $s$.

Lemma 4.3 ([9]). Assume that $\varphi$ satisfies (4.2) where $\psi$ satisfies Assumption $\mathrm{A}^{\prime}$ and (iv) of Assumption $\mathrm{A}$ and the Lamé coefficients satisfy (4.7). Let $\mathbf{f}=\tilde{\mathbf{f}}+\sum_{j=0}^{2} \partial_{x_{j}} \mathbf{f}_{j}$ with $\tilde{\mathbf{f}} \in L^{2}\left(0, T ; \mathbf{H}^{-1}(\Omega)\right)$ and $\mathbf{f}_{0}, \mathbf{f}_{1}, \mathbf{f}_{2} \in \mathbf{L}^{2}(Q)$. Then there exists $\widehat{\tau}>0$ such that for any $\tau>\widehat{\tau}$, there exists $s_{0}=s_{0}(\tau)>0$ such that for any solution $\mathbf{u} \in \mathbf{L}^{2}(Q)$ 
to problem (4.5)-(4.6), the following estimate is valid for all $s \geq s_{0}(\tau)$ :

$$
\int_{Q}|\mathbf{u}|^{2} \mathrm{e}^{2 s \varphi} \mathrm{d} x \leq C_{3}\left(\left\|\tilde{\mathbf{f}}^{s \varphi}\right\|_{L^{2}\left(0, T ; \mathbf{H}^{-1}(\Omega)\right)}^{2}+\sum_{j=0}^{2}\left\|\mathbf{f}_{j} \mathrm{e}^{s \varphi}\right\|_{\mathbf{L}^{2}(Q)}^{2}+\int_{Q_{\omega}}|\mathbf{u}|^{2} \mathrm{e}^{2 s \varphi} \mathrm{d} x\right)
$$

where the constant $C_{3}=C_{3}(\tau)>0$ is independent of $s$.

Consider the boundary value problem for the Laplace operator

$$
\Delta_{x^{\prime}} u=\tilde{f}+\sum_{j=1}^{2} \partial_{x_{j}} \tilde{f}_{j} \quad \text { in } \Omega,\left.\quad u\right|_{\partial \Omega}=\tilde{g}
$$

The lemma below refers to a function $\varphi(x)=\mathrm{e}^{\tau \psi(x)}$, where $\tau$ is a large parameter and $\psi$ is described in the lemma.

Lemma $4.4([8])$. Let $\tilde{g} \in L^{2}\left(0, T ; H^{\frac{1}{2}}(\Gamma)\right), \tilde{f} \in L^{2}\left(0, T ; L^{2}(\Omega)\right), \tilde{f}_{j} \in L^{2}\left(0, T ; L^{2}(\Omega)\right)$. Let $\psi(x) \in C^{2}(\bar{Q})$, $\partial_{\vec{n}} \psi<0$ on $(0, T) \times \Gamma$ and $\left|\nabla_{x^{\prime}} \psi\right| \neq 0$ on $[0, T] \times \overline{\Omega \backslash \omega}$. Then solutions to (4.14) satisfy the estimate

$$
\begin{aligned}
&\left\|\nabla_{x^{\prime}} u \mathrm{e}^{s \varphi}\right\|_{L^{2}(Q)}+s\left\|u \mathrm{e}^{s \varphi}\right\|_{L^{2}(Q)} \leq C\left(s^{\frac{1}{4}}\left\|\tilde{g} \mathrm{e}^{s \varphi}\right\|_{L^{2}\left(0, T ; H^{\left.\frac{1}{2}(\Gamma)\right)}\right.}+\frac{1}{\sqrt{s}}\left\|\tilde{f} \mathrm{e}^{s \varphi}\right\|_{L^{2}(Q)}\right. \\
&\left.+\sqrt{s} \sum_{j=1}^{2}\left\|\tilde{f}_{j} \mathrm{e}^{s \varphi}\right\|_{L^{2}(Q)}+\left\|\nabla_{x^{\prime}} u \mathrm{e}^{s \varphi}\right\|_{L^{2}\left(Q_{\omega}\right)}+s\left\|u \mathrm{e}^{s \varphi}\right\|_{L^{2}\left(Q_{\omega}\right)}\right) \quad \forall s \geq s_{0}, \\
& s\left\|u \mathrm{e}^{s \varphi}\right\|_{L^{2}(Q)} \leq C\left(s^{\frac{3}{4}}\left\|\tilde{g} \mathrm{e}^{s \varphi}\right\|_{L^{2}(\Sigma)}+\frac{1}{\sqrt{s}}\left\|\tilde{f} \mathrm{e}^{s \varphi}\right\|_{L^{2}(Q)}+\sqrt{s} \sum_{j=1}^{2}\left\|\tilde{f}_{j} \mathrm{e}^{s \varphi}\right\|_{L^{2}(Q)}+s\left\|u \mathrm{e}^{s \varphi}\right\|_{L^{2}\left(Q_{\omega}\right)}\right) \quad \forall s \geq s_{0},
\end{aligned}
$$

where $C=C(\tau)>0$ is independent of $s$.

\subsection{Proof of main results for clamped boundary conditions}

Next we consider the partial differential equation

$$
\begin{gathered}
\mathcal{K}(D) v=m \partial_{x_{0}}^{2} v-\alpha \Delta_{x^{\prime}} \partial_{x_{0}}^{2} v+K \Delta_{x^{\prime}}^{2} v=f \text { in } Q \\
\left.v\right|_{\Sigma}=0,\left.\partial_{\vec{n}} v\right|_{\Sigma}=0, v(0, \cdot)=v(T, \cdot)=\partial_{x_{0}} v(0, \cdot)=\partial_{x_{0}} v(T, \cdot)=0 .
\end{gathered}
$$

We introduce the Banach spaces

$$
X=\left\{v(x), x \in Q: v, \nabla v \in L^{2}\left(0, T ; H^{1}(\Omega)\right)\right\}
$$

with norm $\|v\|_{X}=\|\nabla v\|_{L^{2}\left(0, T ; H^{1, s}(\Omega)\right)}+s\|\nabla v\|_{L^{2}\left(0, T ; L^{2}(\Omega)\right)}+s^{2}\|v\|_{L^{2}(Q)}$ and $X^{*}$ is the space of the linear continuous functionals on $X$. Also define $H^{1, s}(\Omega)=H^{1}(\Omega)$ with norm $\|v\|_{H^{1, s}(\Omega)}=\|v\|_{H^{1}(\Omega)}+s\|v\|_{L^{2}(\Omega)}$.

We have:

Lemma 4.5. Let $f \in X^{*}$ and $\varphi$ satisfy (4.2) where $\psi \in C^{2}(\bar{Q})$ is pseudoconvex with respect to the symbol $\xi_{0}^{2}-\frac{K}{\alpha}\left(\xi_{1}^{2}+\xi_{2}^{2}\right), \partial_{\vec{n}} \psi<0$ on $(0, T) \times \Gamma$ and $\left|\nabla_{x^{\prime}} \psi\right| \neq 0$ on $[0, T] \times \overline{\Omega \backslash \omega}$. Then there exists $s_{0}>0$ such that for all solutions to problem (4.17)-(4.18) the following estimate holds true

$$
\left\|v \mathrm{e}^{s \varphi}\right\|_{L^{2}\left(0, T ; H^{1, s}(\Omega)\right)} \leq C\left(\left\|f \mathrm{e}^{s \varphi}\right\|_{X^{*}}+\left\|v \mathrm{e}^{s \varphi}\right\|_{H^{1, s}\left(Q_{\omega}\right)}\right) \quad \forall s \geq s_{0},
$$

where $C=C(\tau)>0$ is independent of $s$. 
Proof. First we prove the Carleman estimate (4.19) for solutions of the problem (4.17)-(4.18) in slightly different spaces:

$$
\sum_{|\alpha|=0}^{2} s^{2-|\alpha|}\left\|D^{\alpha} v \mathrm{e}^{s \varphi}\right\|_{L^{2}(Q)} \leq C\left(\left\|f \mathrm{e}^{s \varphi}\right\|_{L^{2}\left(0, T ;\left(H^{1, s}(\Omega)\right)^{*}\right)}+\sum_{|\alpha|=0}^{2} s^{2-|\alpha|}\left\|D^{\alpha} v \mathrm{e}^{s \varphi}\right\|_{L^{2}\left(Q_{\omega}\right)}\right) \quad \forall s \geq s_{0}(\tau) .
$$

Here the space $\left(H^{1, s}(\Omega)\right)^{*}$ is the space $\left(H^{1}(\Omega)\right)^{*}$ equipped with the norm

$$
\|\tilde{f}\|_{\left(H^{1, s}(\Omega)\right)^{*}}=\sup _{z \in H^{1, s}(\Omega),\|z\|_{H^{1, s}(\Omega)}=1}|<\tilde{f}, z>| .
$$

By the Riesz representation theorem each $f$ in $\left(H^{1}\right)^{*}$ can be represented uniquely as an element of $H^{1}$ as follows:

$$
\langle f, \delta\rangle=\int_{\Omega} f \delta+\partial_{x_{1}} f \partial_{x_{1}} \delta+\partial_{x_{2}} f \partial_{x_{2}} \delta \mathrm{d} x^{\prime} \quad \forall \delta \in H^{1}(\Omega) .
$$

Therefore there exists $L^{2}(\Omega)$ functions $f_{i}, i=0,1,2$ such that

$$
\langle f, \delta\rangle=\int_{\Omega} f_{0} \delta-f_{1} \partial_{x_{1}} \delta-f_{2} \partial_{x_{2}} \delta \mathrm{d} x^{\prime} \quad \forall \delta \in H^{1}(\Omega) .
$$

Of course for any $f_{i}$ in $L^{2}(\Omega)$ the functional given by (4.21) remains continuous on $H^{1}(\Omega)$.

By density of test functions in $L^{2}(\Omega)$ we can approximate $f_{i}$ by $f_{i, \epsilon} \in C_{0}^{\infty}(\Omega)$ such that $\left\|f_{i, \epsilon}-f_{i}\right\|_{L^{2}(\Omega)} \leq \epsilon / 3$ for $i=0,1,2$. Define $f_{\epsilon}$ by $f_{\epsilon}=f_{0, \epsilon}+\partial_{x_{1}} f_{1, \epsilon}+\partial_{x_{2}} f_{2, \epsilon}$. Define the following linear functional on $H^{1}(\Omega)$ :

$$
\left\langle f_{\epsilon}, \delta\right\rangle=\int_{\Omega} f_{0, \epsilon} \delta-f_{1, \epsilon} \partial_{x_{1}} \delta-f_{2, \epsilon} \partial_{x_{2}} \delta \mathrm{d} x^{\prime}=\int_{\Omega} f_{\epsilon} \delta \mathrm{d} x^{\prime} \quad \forall \delta \in H^{1}(\Omega) .
$$

Since

$$
\begin{aligned}
\left|\left\langle f-f_{\epsilon}, \delta\right\rangle\right| & \leq \int_{\Omega}\left|f_{0}-f_{0, \epsilon}\right||\delta|+\left|f_{1}-f_{1, \epsilon}\right|\left|\partial_{x_{1}} \delta\right|+\left|f-f_{2, \epsilon}\right|\left|\partial_{x_{2}} \delta\right| \mathrm{d} x^{\prime} \quad \forall \delta \in H^{1}(\Omega) \\
& \leq \epsilon\|\delta\|_{H^{1}(\Omega)}
\end{aligned}
$$

$f_{\epsilon} \rightarrow f$ in $H^{1}(\Omega)^{*}$. Now consider the scalar product for the space $L^{2}\left(0, T ; H^{1, s}(\Omega)\right)$ :

$$
(u, v)=\int_{Q}\left(\sum_{k=1}^{2} \partial_{x_{k}} v \partial_{x_{k}} u+s u v\right) \mathrm{d} x^{\prime} \mathrm{d} x_{0}
$$

By the Riesz representation theorem, each element $x^{*}$ of $L^{2}\left(0, T ;\left(H^{1, s}(\Omega)\right)\right)^{*}$ is represented as an element $f$ of $L^{2}\left(0, T ;\left(H^{1, s}(\Omega)\right)^{*}\right)$, which can be approximated (as above) by $C_{0}^{\infty}(Q)$ functions $f_{\epsilon}=\sum_{i=1}^{2} \partial_{x_{i}} f_{i, \epsilon}+f_{0, \epsilon}$ such that $f_{i, \epsilon} \in C_{0}^{\infty}(Q)$ and $f_{i, \epsilon} \rightarrow f_{i}$ in $L^{2}\left(0,2 T ; L^{2}(\Omega)\right)$ for all $i \in\{0,1,2\}$ assuming that we define $f_{i}\left(x_{0}, \cdot\right) \equiv 0$ for $x_{0}>T$.

Let $v_{\epsilon}$ be the solution to the problem

$$
\mathcal{K}(D) v_{\epsilon}=f_{\epsilon},\left.\quad v_{\epsilon}\right|_{\partial \Omega}=\left.\partial_{\vec{n}} v_{\epsilon}\right|_{\partial \Omega}=0, \quad v_{\epsilon}(0, \cdot)=\partial_{x_{0}} v_{\epsilon}(0, \cdot)=0 .
$$

Then, due to e.g., [16], $v_{\epsilon} \rightarrow v$ in $H^{2}((0,2 T) \times \Omega)$. Let $\chi \in C^{\infty}\left(\mathbb{R}^{1}\right)$ be a function such that $\chi\left(x_{0}\right)=1$ for $x_{0} \leq 0$ and $\chi\left(x_{0}\right)=0$ for $x_{0} \geq 1$. We set $\chi_{\epsilon_{1}}=\chi\left(\left(x_{0}-T\right) / \epsilon_{1}\right)$ and $\tilde{v}_{\epsilon, \epsilon_{1}}=\chi_{\epsilon_{1}} v_{\epsilon}$. Obviously we have

$$
\begin{aligned}
& \mathcal{K}(D) v_{\epsilon, \epsilon_{1}}=\chi_{\epsilon_{1}} f_{\epsilon}-\left[\chi_{\epsilon_{1}}, \mathcal{K}\right] v_{\epsilon},\left.\quad v_{\epsilon}\right|_{\left(0, T+\epsilon_{1}\right) \times \Gamma}=\left.\partial_{\vec{n}} v_{\epsilon}\right|_{\left(0, T+\epsilon_{1}\right) \times \Gamma}=0, \\
& v_{\epsilon, \epsilon_{1}}(0, \cdot)=\partial_{x_{0}} v_{\epsilon, \epsilon_{1}}(0, \cdot)=v_{\epsilon, \epsilon_{1}}\left(T+\epsilon_{1}, \cdot\right)=\partial_{x_{0}} v_{\epsilon, \epsilon_{1}}\left(T+\epsilon_{1}, \cdot\right)=0 .
\end{aligned}
$$


Since there exist subsequences $\epsilon_{0, k}, \epsilon_{1, k}$ such that $\left\|\left[\chi_{\epsilon_{1, k}}, \mathcal{K}\right] v_{\epsilon_{0, k}}\right\|_{L^{2}\left(0, T ;\left(H^{1}(\Omega)\right)^{*}\right)} \rightarrow 0$ as $\epsilon_{0, k}, \epsilon_{1_{k}} \rightarrow+0$ it suffices to prove Carleman (4.20) estimate only for $f \in C^{\infty}\left(0, T ; C_{0}^{\infty}(\bar{\Omega})\right)$. Suppose that we have proved the following estimate:

$$
\sqrt{s}\left\|\partial_{\bar{n}}^{2} v \mathrm{e}^{s \varphi}\right\|_{L^{2}(\Sigma)} \leq C\left(\left\|f \mathrm{e}^{s \varphi}\right\|_{L^{2}\left(0, T ;\left(H^{1, s}(\Omega)\right)^{*}\right)}+\sum_{|\alpha|=0}^{2} s^{2-|\alpha|}\left\|D^{\alpha} v \mathrm{e}^{s \varphi}\right\|_{L^{2}\left(Q_{\omega}\right)}\right) \quad \forall s \geq s_{0}(\tau) .
$$

Then applying the Carleman estimates (4.16) and (4.3) to equation (4.17) we have

$$
\begin{aligned}
\sqrt{s}\left(\left\|Q(\widetilde{\mathbf{D}})\left(v \mathrm{e}^{s \varphi}\right)\right\|_{L^{2}(Q)}+\left\|\Delta_{x^{\prime}}(\widetilde{\mathbf{D}})\left(v \mathrm{e}^{s \varphi}\right)\right\|_{L^{2}(Q)}\right) \leq & C\left(\left\|\left(f-m \partial_{x_{0}}^{2} v\right) \mathrm{e}^{s \varphi}\right\|_{L^{2}\left(0, T ;\left(H^{1, s}(\Omega)\right)^{*}\right)}+\sqrt{s}\left\|\partial_{\vec{n}}^{2} v \mathrm{e}^{s \varphi}\right\|_{L^{2}(\Sigma)}\right. \\
& \left.+\sum_{|\alpha|=0}^{2} s^{2-|\alpha|}\left\|D^{\alpha} v \mathrm{e}^{s \varphi}\right\|_{L^{2}\left(Q_{\omega}\right)}\right) \quad \forall s \geq s_{0}(\tau),
\end{aligned}
$$

where

$$
Q(\widetilde{\mathbf{D}})=\left(\frac{1}{i} \partial_{x_{0}}+i s \partial_{x_{0}} \varphi\right)^{2}-\frac{K}{\alpha} \Delta_{x^{\prime}}(\widetilde{\mathbf{D}}), \quad \Delta_{x^{\prime}}(\widetilde{\mathbf{D}})=\left(\frac{1}{i} \partial_{x_{1}}+i s \partial_{x_{1}} \varphi\right)^{2}+\left(\frac{1}{i} \partial_{x_{2}}+i s \partial_{x_{2}} \varphi\right)^{2} .
$$

Thanks to (4.23)

$$
\begin{aligned}
\sqrt{s}\left(\left\|Q(\widetilde{\mathbf{D}})\left(v \mathrm{e}^{s \varphi}\right)\right\|_{L^{2}(Q)}+\left\|\Delta_{x^{\prime}}(\widetilde{\mathbf{D}})\left(v \mathrm{e}^{s \varphi}\right)\right\|_{L^{2}(Q)}\right) \leq C\left(\left\|\left(f-m \partial_{x_{0}}^{2} v\right) \mathrm{e}^{s \varphi}\right\|_{L^{2}\left(0, T ;\left(H^{1, s}(\Omega)\right)^{*}\right)}\right. & \\
& \left.+\sum_{|\alpha|=0}^{2} s^{2-|\alpha|}\left\|D^{\alpha} v \mathrm{e}^{s \varphi}\right\|_{L^{2}\left(Q_{\omega}\right)}\right) \quad \forall s \geq s_{0}(\tau) .
\end{aligned}
$$

Applying the Carleman estimate (4.15) to the elliptic operator $\Delta_{x^{\prime}}(\widetilde{\mathbf{D}})$ in $(4.24)$ we obtain

$$
\begin{gathered}
\sqrt{s}\left(\left\|Q(\widetilde{\mathbf{D}})\left(v \mathrm{e}^{s \varphi}\right)\right\|_{L^{2}(Q)}+\left\|\Delta_{x^{\prime}}(\widetilde{\mathbf{D}})\left(v \mathrm{e}^{s \varphi}\right)\right\|_{L^{2}(Q)}\right)+\sum_{|\alpha|=0, \alpha_{0}=0}^{2} s^{2-|\alpha|}\left\|D^{\alpha} v \mathrm{e}^{s \varphi}\right\|_{L^{2}(Q)} \\
\leq C\left(\left\|\left(f-m \partial_{x_{0}}^{2} v\right) \mathrm{e}^{s \varphi}\right\|_{L^{2}\left(0, T ;\left(H^{1, s}(\Omega)\right)^{*}\right)}+\sum_{|\alpha|=0}^{2} s^{2-|\alpha|}\left\|D^{\alpha} v \mathrm{e}^{s \varphi}\right\|_{L^{2}\left(Q_{\omega}\right)}\right) \quad \forall s \geq s_{0}(\tau) .
\end{gathered}
$$

Then since $\left(\frac{1}{i} \partial_{x_{0}}+i s \partial_{x_{0}} \varphi\right)^{2}=\frac{K}{\alpha} \Delta_{x^{\prime}}(\widetilde{\mathbf{D}})+Q(\widetilde{\mathbf{D}})$, we have

$$
\begin{aligned}
\sqrt{s}\left(\left\|Q(\widetilde{\mathbf{D}})\left(v \mathrm{e}^{s \varphi}\right)\right\|_{L^{2}(Q)}+\right. & \left.\left\|\Delta(\widetilde{\mathbf{D}})\left(v \mathrm{e}^{s \varphi}\right)\right\|_{L^{2}(Q)}+\left\|\left(\partial_{x_{0}}-s \varphi_{x_{0}}\right)^{2}\left(v \mathrm{e}^{s \varphi}\right)\right\|_{L^{2}(Q)}\right)+\sum_{|\alpha|=0, \alpha_{0}=0}^{2} s^{2-|\alpha|}\left\|D^{\alpha} v \mathrm{e}^{s \varphi}\right\|_{L^{2}(Q)} \\
& \leq C\left(\left\|\left(f-m \partial_{x_{0}}^{2} v\right) \mathrm{e}^{s \varphi}\right\|_{L^{2}\left(0, T ;\left(H^{1, s}(\Omega)\right)^{*}\right)}+\sum_{|\alpha|=0}^{2} s^{2-|\alpha|}\left\|D^{\alpha} v \mathrm{e}^{s \varphi}\right\|_{L^{2}\left(Q_{\omega}\right)}\right) \quad \forall s \geq s_{0}(\tau) .
\end{aligned}
$$

This inequality implies

$$
\begin{gathered}
\sqrt{s}\left(\left\|Q(\widetilde{\mathbf{D}})\left(v \mathrm{e}^{s \varphi}\right)\right\|_{L^{2}(Q)}+\left\|\Delta_{x^{\prime}}(\widetilde{\mathbf{D}})\left(v \mathrm{e}^{s \varphi}\right)\right\|_{L^{2}(Q)}\right)+\left\|v \mathrm{e}^{s \varphi}\right\|_{H^{2, s}(Q)} \\
\leq C\left(\left\|\left(f-m \partial_{x_{0}}^{2} v\right) \mathrm{e}^{s \varphi}\right\|_{L^{2}\left(0, T ;\left(H^{1, s}(\Omega)\right)^{*}\right)}+\sum_{|\alpha|=0}^{2} s^{2-|\alpha|}\left\|D^{\alpha} v \mathrm{e}^{s \varphi}\right\|_{L^{2}\left(Q_{\omega}\right)}\right) \quad \forall s \geq s_{0}(\tau) .
\end{gathered}
$$


The term $\left\|m\left(\partial_{x_{0}}^{2} v\right) \mathrm{e}^{s \varphi}\right\|_{L^{2}\left(0, T ;\left(H^{1, s}(\Omega)\right)^{*}\right)}$ can be absorbed by the third term in the left hand side of (4.26). So

$$
\begin{aligned}
& \sqrt{s}\left(\left\|Q(\widetilde{\mathbf{D}})\left(v \mathrm{e}^{s \varphi}\right)\right\|_{L^{2}(Q)}+\left\|\Delta_{x^{\prime}}(\widetilde{\mathbf{D}})\left(v \mathrm{e}^{s \varphi}\right)\right\|_{L^{2}(Q)}\right)+\left\|v \mathrm{e}^{s \varphi}\right\|_{H^{2, s}(Q)} \\
& \quad \leq C\left(\left\|f \mathrm{e}^{s \varphi}\right\|_{L^{2}\left(0, T ;\left(H^{1, s}(\Omega)\right)^{*}\right)}+\sum_{|\alpha|=0}^{2} s^{2-|\alpha|}\left\|D^{\alpha} v \mathrm{e}^{s \varphi}\right\|_{L^{2}\left(Q_{\omega}\right)}\right) \quad \forall s \geq s_{0}(\tau) .
\end{aligned}
$$

Hence (4.23) implies (4.20).

Before we begin the proof of (4.23), it is convenient to define a weight function $\phi$ such that $\left.\phi\right|_{\Gamma}=\left.\varphi\right|_{\Gamma}$ and $\phi(x)<\varphi(x)$ for all $x$ in a neighborhood of $\Sigma$. We construct such a function $\phi$ locally near the boundary $\Gamma$ :

$$
\phi(x)=\mathrm{e}^{\tau \tilde{\psi}(x)}, \quad \widetilde{\psi}(x)=\psi(x)-\frac{1}{\sqrt{N}} \tilde{g}_{1}\left(x^{\prime}\right)+N \tilde{g}_{1}^{2}\left(x^{\prime}\right),
$$

where $N>0$ is a large positive parameter and $\tilde{g}_{1} \in C^{3}(\bar{\Omega})$ satisfies

$$
\tilde{g}_{1}\left(x^{\prime}\right)>0 \quad \forall x^{\prime} \in \Omega,\left.\quad \tilde{g}_{1}\right|_{\Gamma}=0,\left.\quad \nabla_{x^{\prime}} \tilde{g}_{1}\right|_{\Gamma} \neq 0 .
$$

Denote $\Omega_{\frac{1}{N^{2}}}=\left\{x^{\prime} \in \Omega \mid 0<\operatorname{dist}\left(x^{\prime}, \Gamma\right)<\frac{1}{N^{2}}\right\}$. Obviously there exists $N_{0}>0$ such that

$$
\phi(x)<\varphi(x), \quad \forall x \in[0, T] \times \Omega_{\frac{1}{N^{2}}}, N \in\left(N_{0}, \infty\right) .
$$

Now we begin the proof of estimate (4.23). As a first step we prove (4.23) under the assumption supp $v \subset$ $B_{\delta}\left(x^{*}\right)$. We assume that the point $x^{*} \in \Sigma$. (If $B_{\delta}\left(x^{*}\right) \cap \Sigma=\emptyset$ then (4.23) is trivially true. If $B_{\delta}\left(x^{*}\right) \cap \Sigma \neq \emptyset$ then $B_{\delta}\left(x^{*}\right)$ is a subset of a slightly larger ball centered at a point of $\Sigma$.) Without loss of generality we may assume that $x^{*}=0$. Locally near zero the boundary $\Gamma$ is given by equation $x_{2}-g\left(x_{1}\right)=0$. Making the change of variables $y_{1}=x_{1}, y_{2}=x_{2}-g\left(x_{1}\right)$, we obtain

$$
\begin{gathered}
R(y, D) v=\mathcal{Q}(y, D) \mathcal{A}(y, D) v=\mathcal{A}(y, D) \mathcal{Q}(y, D) v=f \quad \text { in } \mathcal{G}, \\
\left.v\right|_{y_{2}=0}=\left.\partial_{y_{2}} v\right|_{y_{2}=0}=0,
\end{gathered}
$$

where

$$
\mathcal{G}=\left\{\left(y_{0}, y_{1}, y_{2}\right) \in \mathbb{R}^{2} \times \mathbb{R}^{+}\right\}
$$

and $\mathcal{Q}(y, D)=-D_{y_{0}}^{2}+\mathcal{A}(y, D)$, and $\mathcal{A}$ is the Laplace operator after the change of variables, which is extended outside of the image of $B_{\delta}(0)$ to an elliptic operator on $\mathcal{G}$. Denote $w(y)=v(y) \mathrm{e}^{|s| \phi(y)}$, where $\phi(y)$ is the function $\phi$ after change of the variables and let $\mathbf{D}_{y_{k}}=\left(\frac{1}{i} \partial_{y_{k}}+i|s| \phi_{y_{k}}\right)$, where $\mathbf{D}=\left(\mathbf{D}_{y_{0}}, \mathbf{D}_{y_{1}}, \mathbf{D}_{y_{2}}\right)$. Then we have

$$
\begin{gathered}
R(y, \mathbf{D}) w=\mathcal{Q}(y, \mathbf{D}) \mathcal{A}(y, \mathbf{D}) w=\mathcal{A}(y, \mathbf{D}) \mathcal{Q}(y, \mathbf{D}) w=f \mathrm{e}^{|s| \phi} \quad \text { in } \mathcal{G}, \\
\left.w\right|_{y_{2}=0}=\left.\partial_{y_{2}} w\right|_{y_{2}=0}=0,
\end{gathered}
$$

with $\operatorname{supp} w \subset B_{\tilde{\delta}}\left(y^{*}\right)$, where $B_{\tilde{\delta}}\left(y^{*}\right)$ contains the image of $B_{\delta}(0)$ after the change of variables.

Denote by $a(y, \xi)$ the principal symbol of the operators $\mathcal{A}(y, D)$ :

$$
a(y, \xi)=\xi_{1}^{2}-2 g^{\prime}\left(y_{1}\right) \xi_{1} \xi_{2}+\left(1+\left|g^{\prime}\left(y_{1}\right)\right|^{2}\right) \xi_{2}^{2} .
$$

We cover the sphere $S^{2}$ by small balls $B_{\delta_{1}}\left(\xi_{0, \nu}, \xi_{1, \nu}, s_{\nu}\right)$, and let $\left\{\chi_{\nu}\right\}$ be the partition of unity applied to this covering: $\sum_{\nu} \chi_{\nu}=1, \chi_{\nu} \geq 0, \chi_{\nu} \in C_{0}^{\infty}\left(B_{\delta_{1}}\left(\xi_{0, \nu}, \xi_{1, \nu}, s_{\nu}\right)\right)$. We extend the function $\chi_{\nu}$ as a homogeneous function of order 0 on the set $\left|\left(\xi_{0}, \xi_{1}, s\right)\right| \geq 1$ and up to a $C^{\infty}$ function on $\mathbb{R}^{3}$. Let $\gamma=\left(y^{*}, \xi_{0}^{*}, \xi_{1}^{*}, s^{*}\right)$, where $\left(\xi_{0}^{*}, \xi_{1}^{*}, s^{*}\right)$ is an arbitrary point of $S^{2}$ and $w_{\nu}=\chi_{\nu}\left(D^{\prime}, s\right) w, D^{\prime}=\left(\frac{1}{i} \partial_{y_{0}}, \frac{1}{i} \partial_{y_{1}}\right)$. 
Denote $w_{\nu}(y)=\chi_{\nu}\left(D_{y_{0}}, D_{y_{1}}, s\right)\left(v(y) \mathrm{e}^{|s| \phi(y)}\right), q=\chi_{\nu}\left(f \mathrm{e}^{|s| \phi}\right)-\left[\chi_{\nu}, R(y, \mathbf{D})\right]$. Then we have

$$
\begin{gathered}
R(y, \mathbf{D}) w_{\nu}=\mathcal{Q}(y, \mathbf{D}) \mathcal{A}(y, \mathbf{D}) w_{\nu}=\mathcal{A}(y, \mathbf{D}) \mathcal{Q}(y, \mathbf{D}) w_{\nu}=q \quad \text { in } \mathcal{G} \\
\left.w_{\nu}\right|_{y_{2}=0}=0,\left.\quad \partial_{y_{2}} w_{\nu}\right|_{y_{2}=0}=0
\end{gathered}
$$

with $\operatorname{supp} w \subset B_{\tilde{\delta}}\left(y^{*}\right)$. Note that $[\mathcal{Q}(y, \mathbf{D}), \mathcal{A}(y, \mathbf{D})]=0$.

Let us consider the case when $\left(\xi_{1}^{*}, s^{*}\right) \neq 0$. We factor the operator $\mathcal{A}(y, \mathbf{D})$ as follows:

$$
\mathcal{A}(y, \mathbf{D})=\mathcal{A}_{-}(y, D, s) \mathcal{A}_{+}(y, D, s)+T\left(y_{2}\right)=\mathcal{A}_{+}(y, D, s) \mathcal{A}_{-}(y, D, s)+\tilde{T}\left(y_{2}\right),
$$

where $T\left(y_{2}\right), \tilde{T}\left(y_{2}\right)$ are the operators such that for some $\kappa>\tilde{\delta}$,

$$
\tilde{T}\left(y_{2}\right), T\left(y_{2}\right) \in C^{2}\left(0, \kappa ; \mathcal{L}\left(H^{k, s}\left(\mathbb{R}^{3}\right) ; H^{k-1, s}\left(\mathbb{R}^{3}\right)\right)\right), \quad k=1,2 .
$$

Let us write $\xi^{\prime}=\left(\xi_{0}, \xi_{1}\right)$ and set

$$
\begin{aligned}
r_{\mathcal{A}}\left(y, \xi_{1}, s\right) & =-\left(\xi_{1}+i|s| \phi_{y_{1}}\right)^{2} \\
r_{\mathcal{Q}}\left(y, \xi^{\prime}, s\right) & =\frac{\alpha}{K}\left(\xi_{0}+i|s| \phi_{y_{0}}\right)^{2}+r_{\mathcal{A}}\left(y, \xi_{1}, s\right)
\end{aligned}
$$

and

$$
\begin{aligned}
\Gamma_{\mathcal{Q}}^{ \pm}\left(y, \xi^{\prime}, s\right) & =-|s| \phi_{y_{2}} i+\frac{g^{\prime}\left(y_{1}\right)\left(\xi_{1}+i|s| \phi_{y_{1}}\right)+{\sqrt{r_{\mathcal{Q}}}}^{ \pm}\left(y, \xi^{\prime}, s\right)}{\left(1+\left(g^{\prime}\left(y_{1}\right)\right)^{2}\right)} \\
\Gamma_{\mathcal{A}}^{ \pm}\left(y, \xi^{\prime}, s\right) & =-|s| \phi_{y_{2}} i+\frac{g^{\prime}\left(y_{1}\right)\left(\xi_{1}+i|s| \phi_{y_{1}}\right)+{\sqrt{r_{\mathcal{A}}}}^{ \pm}\left(y, \xi_{1}, s\right)}{\left(1+\left(g^{\prime}\left(y_{1}\right)\right)^{2}\right)}
\end{aligned}
$$

Here $\sqrt{ }^{ \pm}$refers to the branch of the complex root with positive or negative imaginary part. When $\left(\xi_{1}^{*}, s^{*}\right) \neq 0$ explicitly we have

$$
{\sqrt{r_{\mathcal{A}}}}^{ \pm}\left(y, \xi_{1}, s\right)= \begin{cases} \pm i \operatorname{sign}\left(\xi_{1}^{*}\right)\left(\xi_{1}+i|s| \phi_{y_{1}}\right) & \text { if } \quad \xi_{1}^{*} \neq 0 \\ \pm i\left(\xi_{1}+i|s| \phi_{y_{1}}\right) & \text { if } \quad \xi_{1}^{*}=0\end{cases}
$$

The principal symbols of operators $\mathcal{A}_{ \pm}$in (4.37) are given by

$$
\mathcal{A}_{ \pm}(y, \xi, s)=\xi_{2}+\Gamma_{\mathcal{A}}^{ \pm}\left(y, \xi^{\prime}, s\right) .
$$

We set $z_{1}=\mathcal{A}_{+}(y, D, s)\left(\mathcal{Q}(y, \mathbf{D}) w_{\nu}\right)$. Thanks to (4.35), (4.36), (4.37) this function satisfies the equation

$$
\mathcal{A}_{-}(y, D, s) z_{1}=q-T \mathcal{Q}(y, \mathbf{D}) w_{\nu} \quad \text { in } \mathcal{G},\left.\quad z_{1}\right|_{y_{2}=\kappa}=0,
$$

with

$$
\left\|q-T \mathcal{Q}(y, \mathbf{D}) w_{\nu}\right\|_{\mathcal{Z}} \leq C\left(\|q\|_{\mathcal{Z}}+\left\|w_{\nu}\right\|_{H^{2, s}(\mathcal{G})}\right)
$$

where $\mathcal{Z}$ is a dual space to $\tilde{\mathcal{Z}}=\left\{w: w, \partial_{y_{1}} w, \partial_{y_{2}} w \in L^{2}(\mathcal{G})\right\}$ equipped with the norm $\|w\|_{\tilde{\mathcal{Z}}}=s\|w\|_{L^{2}(\mathcal{G})}+$ $\left\|\partial_{y_{1}} w\right\|_{L^{2}(\mathcal{G})}+\left\|\partial_{y_{2}} w\right\|_{L^{2}(\mathcal{G})}$.

For the initial value problem (4.41) the following a priori estimate holds (see e.g., Prop. 2.3 of [11]):

$$
\left\|\mathcal{A}_{+}(y, D, s) \mathcal{Q}(y, \mathbf{D}) w_{\nu}\right\|_{L^{2}(\mathcal{G})} \leq C\left(\|q\|_{\mathcal{Z}}+\left\|w_{\nu}\right\|_{H^{2, s}(\mathcal{G})}\right) .
$$

Next we consider the following boundary value problem with $z_{2}=\mathcal{A}_{+}(y, D, s) w_{\nu}$ treated as the unknown:

$$
\mathcal{Q}(y, \mathbf{D}) z_{2}=-\left[\mathcal{A}_{+}, \mathcal{Q}(y, \mathbf{D})\right] w_{\nu}+\mathcal{A}_{+}(y, D, s) \mathcal{Q}(y, \mathbf{D}) w_{\nu}, z_{2}(\cdot, 0)=z_{2}(\cdot, \kappa)=\partial_{y_{2}} z_{2}(\cdot, \kappa)=0 .
$$


Since operator $\mathcal{Q}(y, D)$ is hyperbolic and the pseudoconvexity property is preserved under the change of variables we may apply to the above equations the Carleman estimate for the hyperbolic equations (from Lem. 4.1) to obtain

$$
\sqrt{|s|}\left\|\partial_{y_{2}}^{2} w_{\nu}(\cdot, 0)\right\|_{L^{2}\left(\mathbb{R}^{2}\right)} \leq C\left(\|q\|_{\mathcal{Z}}+\|w\|_{H^{2, s}(\mathcal{G})}\right) .
$$

Next we consider the case when $\left(\xi_{1}^{*}, s^{*}\right)=0$.

Since $\left(\xi_{0}^{*}, \xi_{1}^{*}, s^{*}\right) \neq 0$ then $r_{\mathcal{Q}}(\gamma) \neq 0$ and we can factor the operator $\mathcal{Q}$ into the product of two operators and a remainder as

$$
\mathcal{Q}(y, \mathbf{D})=\mathcal{Q}_{-}(y, D, s) \mathcal{Q}_{+}(y, D, s)+T_{+}\left(y_{2}\right)=\mathcal{Q}_{+}(y, D, s) \mathcal{Q}_{-}(y, D, s)+T_{-}\left(y_{2}\right)
$$

where the principal symbol of the operator $\mathcal{Q}_{ \pm}(y, D, s)$ is

$$
\mathcal{Q}_{ \pm}(y, \xi, s)=\sqrt{1+\left(g^{\prime}\left(y_{1}\right)\right)^{2}}\left(\xi_{2}-\Gamma_{\mathcal{Q}}^{ \pm}\left(y, \xi^{\prime}, s\right)\right)
$$

and operators $T_{ \pm}$satisfy

$$
T_{ \pm} \in \mathcal{L}\left(H^{k+1, s}(\mathcal{G}), H^{k, s}(\mathcal{G})\right) \quad k \in\{1,2\}
$$

We set $z_{2}=\mathcal{Q}_{+}(y, D, s)\left(\mathcal{A}(y, \mathbf{D}) w_{\nu}\right)$. Thanks to $(4.35)$, (4.36) this function satisfies the equation

$$
\mathcal{Q}_{-}(y, D, s) z_{2}=q-T_{+} \mathcal{A}(y, \mathbf{D}) w_{\nu} \quad \text { in } \mathcal{G},\left.\quad z_{2}\right|_{y_{2}=\kappa}=0
$$

with

$$
\left\|q-T_{+} \mathcal{Q}(y, \mathbf{D}) w_{\nu}\right\|_{\mathcal{Z}} \leq C\left(\|q\|_{\mathcal{Z}}+\left\|w_{\nu}\right\|_{H^{2, s}(\mathcal{G})}\right) .
$$

For the initial value problem (4.47) the following a priori estimate holds:

$$
\sqrt{|s|}\left\|\mathcal{Q}_{+}(y, D, s) \mathcal{A}(y, \mathbf{D}) w_{\nu}(\cdot, 0)\right\|_{H^{-1, s}\left(\mathbb{R}^{2}\right)} \leq C\left(\|q\|_{\mathcal{Z}}+\left\|w_{\nu}\right\|_{H^{2, s}(\mathcal{G})}\right)
$$

A small inconvenience is that the function $\left|\xi_{1}\right|$ is not smooth when $\xi_{1}=0$, so the symbol ${\sqrt{r_{\mathcal{A}}}}^{ \pm}\left(y, \xi_{1}, s\right)$ in (4.39) is not continuously dependent on $\xi_{1}^{*}$. In order to overcome this difficulty we consider the covering of $S^{1}=\left\{\left(\xi_{1}, s\right): s^{2}+\xi_{1}^{2}=1\right\}$ by the following set: $e_{1}=\left\{\left(\xi_{1}, s\right) \in S^{1}:\left|\xi_{1}\right| \leq 3 \epsilon|s|\right\}$ and $e_{2}=\left\{\left(\xi_{1}, s\right) \in S^{1}\right.$ : $\left.\left|\xi_{1}\right| \geq \epsilon|s|\right\}$ with some small positive $\epsilon$. Next we consider the partition of unity submitted to this covering of $S^{1}$ : $\tilde{\chi}_{i} \in C_{0}^{\infty}\left(e_{i}\right), \tilde{\chi}_{1}\left(\xi_{1}, s\right)+\tilde{\chi}_{2}\left(\xi_{1}, s\right)=1$. We extend the functions $\tilde{\chi}_{i}$ on the set $\left\{\xi_{1}^{2}+s^{2} \geq 1\right\}$ as homogeneous functions of order zero. Also we extend the functions $\tilde{\chi}_{i}$ on the set $\left\{\xi_{1}^{2}+s^{2} \leq 1\right\}$ as smooth functions. By $\tilde{\chi}_{k}\left(D_{y_{1}}, s\right)$ we denote the pseudodifferential operators with symbols $\tilde{\chi}_{k}\left(\xi_{1}, s\right)$.

For $k=1,2$ we set $z_{1, k}=\mathcal{A}_{+}(y, D, s) \tilde{\chi}_{k}\left(D_{y_{1}}, s\right)\left(\mathcal{Q}(y, \mathbf{D}) w_{\nu}\right)$. Thanks to $(4.35),(4.36)$ this function satisfies the equation

$$
\mathcal{A}_{-}(y, D, s) z_{1, k}=\tilde{\chi}_{k} q-T_{+} \tilde{\chi}_{k}\left(D_{y_{1}}, s\right) \mathcal{Q}(y, \mathbf{D}) w_{\nu}+\left[\mathcal{A}(y, \mathbf{D}), \tilde{\chi}_{k}\right] \mathcal{Q}(y, \mathbf{D}) w_{\nu} \quad \text { in } \mathcal{G}
$$

with $\left.z_{1, k}\right|_{y_{2}=\kappa}=0$ and hence by $(4.46)$

$$
\left\|\tilde{\chi}_{k} q-T_{+} \tilde{\chi}_{k}\left(D_{y_{1}}, s\right) \mathcal{Q}(y, \mathbf{D}) w_{\nu}+\left[\mathcal{A}(y, \mathbf{D}), \tilde{\chi}_{k}\left(D_{y_{1}}, s\right)\right] \mathcal{Q}(y, \mathbf{D}) w_{\nu}\right\|_{\mathcal{Z}} \leq C\left(\|q\|_{\mathcal{Z}}+\left\|w_{\nu}\right\|_{H^{2, s}(\mathcal{G})}\right)
$$

Then we have

$$
\begin{array}{r}
\left\|\mathcal{A}_{+}(y, D, s) \tilde{\chi}_{k}\left(D_{y_{1}}, s\right) \mathcal{Q}(y, \mathbf{D}) w_{\nu}\right\|_{L^{2}(\mathcal{G})}+\left\|\Lambda^{-\frac{1}{2}}\left(D_{1}, s\right) \mathcal{A}_{+}(y, D, s) \tilde{\chi}_{k}\left(D_{1}, s\right) \mathcal{Q}(y, \mathbf{D}) w_{\nu}(\cdot, 0)\right\|_{L^{2}\left(\mathbb{R}^{2}\right)} \\
\leq C\left(\|q\|_{\mathcal{Z}}+\left\|w_{\nu}\right\|_{H^{2, s}(\mathcal{G})}\right)
\end{array}
$$

From (4.52) and (4.49) (again using Prop. 2.3 of [11]) we obtain

$$
\sqrt{|s|}\left\|\partial_{y_{2}}^{2} w_{\nu}(\cdot, 0)\right\|_{L^{2}\left(\mathbb{R}^{2}\right)} \leq C\left(\|q\|_{\mathcal{Z}}+\left\|w_{\nu}\right\|_{H^{2, s}(\mathcal{G})}\right) .
$$


Hence in both cases: $\left(\xi_{1}^{*}, s^{*}\right)=0$ or $\left(\xi_{1}^{*}, s^{*}\right) \neq 0$, we have the same a priori estimate (4.53). Applying the Carleman estimate (4.3) to equation (4.35) and using (4.53) we have

$$
\sqrt{|s|}\left(\left\|\mathcal{A}(y, \mathbf{D}) w_{\nu}\right\|_{L^{2}(\mathcal{G})}+\left\|\mathcal{Q}(y, \mathbf{D}) w_{\nu}\right\|_{L^{2}(\mathcal{G})}+\left\|\partial_{y_{2}}^{2} w_{\nu}(\cdot, 0)\right\|_{L^{2}\left(\mathbb{R}^{2}\right)}\right) \leq C\left(\|q\|_{\mathcal{Z}}+\left\|w_{\nu}\right\|_{H^{2, s}(\mathcal{G})}\right) .
$$

This inequality and the Carleman estimate established in Proposition 5.1 of [9] for the elliptic equation implies

$$
\begin{array}{r}
\sqrt{|s|}\left(\left\|\mathcal{A}(y, \mathbf{D}) w_{\nu}\right\|_{L^{2}(\mathcal{G})}+\left\|\mathcal{Q}(y, \mathbf{D}) w_{\nu}\right\|_{L^{2}(\mathcal{G})}+\left\|\mathbf{D}_{y_{0}}^{2} w_{\nu}\right\|_{L^{2}(\mathcal{G})}\right)+N^{\frac{1}{2}} \sum_{|\alpha|=0, \alpha_{0}=0}^{2}\left\|D^{\alpha} w_{\nu}\right\|_{L^{2}(\mathcal{G})} \\
+\sqrt{|s|}\left\|\partial_{y_{2}}^{2} w_{\nu}(\cdot, 0)\right\|_{L^{2}\left(\mathbb{R}^{2}\right)} \leq C\left(\|q\|_{\mathcal{Z}}+\left\|w_{\nu}\right\|_{H^{2, s}(\mathcal{G})}\right) .
\end{array}
$$

Finally, using the arguments similar to $(4.25)-(4.27)$ we estimate the time $\left(x_{0}\right)$ derivatives of the function $w_{\nu}$ :

$$
\begin{array}{r}
N^{\frac{1}{2}}\left\|w_{\nu}\right\|_{H^{2, s}(\mathcal{G})}+\sqrt{s}\left(\left\|\mathcal{A}(y, \mathbf{D}) w_{\nu}\right\|_{L^{2}(\mathcal{G})}+\left\|\mathcal{Q}(y, \mathbf{D}) w_{\nu}\right\|_{L^{2}(\mathcal{G})}+\left\|\mathbf{D}_{y_{0}}^{2} w_{\nu}\right\|_{L^{2}(\mathcal{G})}\right. \\
\left.+\left\|\partial_{y_{2}}^{2} w_{\nu}(\cdot, 0)\right\|_{L^{2}\left(\mathbb{R}^{2}\right)}\right) \leq C\left(\|q\|_{\mathcal{Z}}+\left\|w_{\nu}\right\|_{H^{2, s}(\mathcal{G})}\right) \quad \forall s \geq s_{0}(\tau, N), N \geq N_{0} .
\end{array}
$$

Increasing the parameter $N_{0}$ if it is necessary we obtain

$$
\begin{array}{r}
N^{\frac{1}{2}}\left\|w_{\nu}\right\|_{H^{2, s}(\mathcal{G})}+\sqrt{s}\left(\left\|\mathcal{A}(y, \mathbf{D}) w_{\nu}\right\|_{L^{2}(\mathcal{G})}+\left\|\mathcal{Q}(y, \mathbf{D}) w_{\nu}\right\|_{L^{2}(\mathcal{G})}\right. \\
\left.+\left\|\mathbf{D}_{y_{0}}^{2} w_{\nu}\right\|_{L^{2}(\mathcal{G})}+\left\|\partial_{y_{2}}^{2} w_{\nu}(\cdot, 0)\right\|_{L^{2}\left(\mathbb{R}^{2}\right)}\right) \leq C\|q\|_{\mathcal{Z}} \quad \forall s \geq s_{0}(\tau, N), N \geq N_{0} .
\end{array}
$$

Hence the following estimate is valid $\forall s \geq s_{0}(\tau, N), N \geq N_{0}$

$$
N^{\frac{1}{2}}\|w\|_{H^{2, s}(\mathcal{G})}+\sqrt{s}\left\|\partial_{y_{2}}^{2} w(\cdot, 0)\right\|_{L^{2}\left(\mathbb{R}^{2}\right)}+s^{\frac{1}{4}} \sum_{|\alpha|=0}^{1}\left\|D^{\alpha} \mathbf{D}_{y_{0}} w_{\nu}\right\|_{L^{2}(\mathcal{G})} \leq C\left\|f \mathrm{e}^{s \phi}\right\|_{\mathcal{Z}}
$$

Consequently estimate (4.23) holds under the assumption that supp $v \subset B_{\delta}\left(x^{*}\right)$. However, by taking $N$ sufficiently large in the above estimate, we no longer need the assumption supp $v \subset B_{\delta}\left(x^{*}\right)$ in the estimate (4.19). This follows using the same argument as in Lemma 8.3.1 of [6].

We now show that (4.20) implies (4.19).

In order to prove this result, we consider the following extremal problem

$$
\begin{gathered}
J\left(z, v_{0}, v_{1}, v_{2}, v_{3}\right)=\frac{1}{2}\left(\left\|\nabla_{x^{\prime}}\left(z \mathrm{e}^{-s \varphi}\right)\right\|_{L^{2}(Q)}^{2}+\left\|z \mathrm{e}^{-s \varphi}\right\|_{L^{2}(Q)}^{2}\right)+\sum_{k=0}^{2} \frac{1}{2}\left\|v_{k} \mathrm{e}^{-s \varphi}\right\|_{L^{2}\left(Q_{\omega}\right)}^{2} \\
+\frac{1}{2 s^{2}}\left\|v_{3} \mathrm{e}^{-s \varphi}\right\|_{L^{2}\left(Q_{\omega}\right)}^{2} \longrightarrow \inf ,
\end{gathered}
$$

$$
\mathcal{K}(D) z=-\mathrm{e}^{s \varphi} \Delta_{x^{\prime}}\left(v \mathrm{e}^{s \varphi}\right)+s^{2} v \mathrm{e}^{2 s \varphi}+\sum_{k=0}^{2} \partial_{x_{0} x_{k}}^{2} v_{k}+v_{3} \quad \text { in } Q,
$$

where $\operatorname{supp} v_{j} \subset \overline{Q_{\omega}}, \quad j=0,1,2,3,\left.\quad v_{k} n_{k}\right|_{\partial \omega \backslash \Gamma}=0, k=1,2$,

$$
\left.\partial_{\vec{n}} z\right|_{\Sigma}=\left.z\right|_{\Sigma}=0, \quad \partial_{x_{0}} z(0, \cdot)=\partial_{x_{0}} z(T, \cdot)=0 .
$$

Remark 4.2. We understand the equalities (4.56)-(4.57) in the weak sense

$$
(z, \mathcal{K}(D) \delta)_{L^{2}(Q)}=\left(\nabla_{x^{\prime}}\left(v \mathrm{e}^{\varphi}\right), \nabla_{x^{\prime}}\left(\delta \mathrm{e}^{\varphi}\right)\right)_{L^{2}(Q)}+\int_{Q}\left(\left(s^{2} v \mathrm{e}^{2 s \varphi}+v_{3}\right) \delta+\sum_{k=0}^{2} v_{k} \partial_{x_{0} x_{k}}^{2} \delta\right) \mathrm{d} x
$$

for all $\delta \in H^{4}(Q)$ such that $\partial_{x_{0}} \delta(0, \cdot)=\partial_{x_{0}} \delta(T, \cdot)=0,\left.\partial_{\vec{n}} \delta\right|_{\Sigma}=\left.\delta\right|_{\Sigma}=0$. 
Denote by $\left(z, v_{0}, v_{1}, v_{2}, v_{3}\right)$ the solution to extremal problem (4.55)-(4.57).

We have:

Lemma 4.6. Under the conditions of Lemma 4.5 for all $v \in L^{2}\left(0, T ; H^{1}(\Omega)\right)$, there exists a unique solution $\left(z, v_{0}, v_{1}, v_{2}, v_{3}\right) \in H^{2}(Q) \times \mathbf{H}^{1}(Q)$ to problem (4.55)-(4.57). Moreover this solution satisfies the optimality system

$$
\begin{array}{r}
\mathcal{K}(D) p-\mathrm{e}^{-s \varphi} \Delta_{x^{\prime}}\left(z \mathrm{e}^{-s \varphi}\right)+s^{2} z \mathrm{e}^{-2 s \varphi}=0 \quad \text { in } Q, \\
\left.\partial_{\vec{n}} p\right|_{\Sigma}=\left.p\right|_{\Sigma}=0, \quad \partial_{x_{0}} p(0, \cdot)=\partial_{x_{0}} p(T, \cdot)=0, \\
p=\frac{1}{s^{2}} v_{3} \mathrm{e}^{-2 s \varphi} \quad \text { in } Q_{\omega}, \quad \partial_{x_{0} x_{k}}^{2} p=v_{k} \mathrm{e}^{-2 s \varphi}, \quad k=0,1,2 \quad \text { in } Q_{\omega}, \\
\mathcal{K}(D) z=-\mathrm{e}^{s \varphi} \Delta_{x^{\prime}}\left(v \mathrm{e}^{s \varphi}\right)+s^{2} v \mathrm{e}^{2 s \varphi}+\sum_{k=0}^{2} \partial_{x_{0} x_{k}}^{2} v_{k}+v_{3}, \quad \text { in } Q, \\
\text { where } \operatorname{supp} v_{j} \subset \overline{Q_{\omega}}, \quad j \in\{0,1,2,3\},\left.\quad v_{k} n_{k}\right|_{\partial \omega \backslash \Gamma}=0, k=1,2 \\
\left.\partial_{\vec{n}} z\right|_{\Sigma}=\left.z\right|_{\Sigma}=0, \quad \partial_{x_{0}} z(0, \cdot)=\partial_{x_{0}} z(T, \cdot)=0,
\end{array}
$$

and there exists $s_{0}>0$ such that the following estimate holds true:

$$
\begin{aligned}
\left\|z \mathrm{e}^{-s \varphi}\right\|_{H^{2, s}(Q)}^{2} & +\sum_{k=0}^{2}\left\|\left(\partial_{x_{0}} v_{k}\right) \mathrm{e}^{-s \varphi}\right\|_{L^{2}\left(Q_{\omega}\right)}^{2}+s^{2}\left\|v_{k} \mathrm{e}^{-s \varphi}\right\|_{L^{2}\left(Q_{\omega}\right)}^{2} \\
& +\left\|v_{3} \mathrm{e}^{-s \varphi}\right\|_{L^{2}\left(Q_{\omega}\right)}^{2} \leq C_{1}\left\|v \mathrm{e}^{s \varphi}\right\|_{H^{1, s}(Q)}^{2} \quad \forall s \geq s_{0},
\end{aligned}
$$

where $C_{1}$ is independent of $s$.

Lemma 4.6 is proved using a standard argument (e.g., [17]) and is similar to one found in [9]. For the sake of completeness, we include the proof in the Appendix.

Now we complete the proof of Lemma 4.5. We plug in (4.58) function $v$ instead of $\delta$. Then we have

$$
\left\|\nabla_{x^{\prime}}\left(v \mathrm{e}^{s \varphi}\right)\right\|_{L^{2}(Q)}^{2}+s^{2}\left\|v \mathrm{e}^{s \varphi}\right\|_{L^{2}(Q)}^{2}=\int_{0}^{T}\langle f, z\rangle \mathrm{d} x_{0}-\int_{Q_{\omega}}\left(\sum_{k=0}^{2} v_{k} \partial_{x_{0} x_{k}}^{2} v+v v_{3}\right) \mathrm{d} x .
$$

Applying (4.64) to this equality the inequality $|a b| \leq \frac{\delta}{2}|a|^{2}+\frac{1}{2 \delta}|b|^{2}$ we obtain

$$
\left\|v \mathrm{e}^{s \varphi}\right\|_{L^{2}\left(0, T ; H^{1, s}(\Omega)\right)}^{2} \leq C_{9}\left(\left\|f \mathrm{e}^{s \varphi}\right\|_{X^{*}}^{2}+\int_{Q_{\omega}}\left(|\nabla v|^{2}+s^{2}|v|^{2}\right) \mathrm{e}^{2 s \varphi} \mathrm{d} x\right), \quad \forall s \geq s_{0}(\tau) .
$$

The proof of the Lemma 4.5 is finished.

Next we consider the system which is formally adjoint to the system (3.1)-(3.5). Let $\mathcal{G}_{E}^{*}=\mathbf{G}_{E}-\tilde{\mathbf{G}}_{E} \partial_{x_{0}}$ then the adjoint system can be written

$$
\begin{gathered}
m \partial_{x_{0}}^{2} q-\alpha \Delta_{x^{\prime}} \partial_{x_{0}}^{2} q+K \Delta_{x^{\prime}}^{2} q-\operatorname{div}_{x^{\prime}} \vec{N}^{T}\left(\mathcal{G}_{E}^{*}\left(B z_{O}+\mathbf{h}_{E} \vec{N} \nabla_{x^{\prime}} q\right)\right)=h \text { on } Q, \\
\mathbf{h}_{O} \mathbf{p}_{O} \partial_{x_{0}}^{2} z_{O}-12 \mathbf{h}_{O} \mathbf{D}_{O} L_{O}(D) z_{O}+B^{T} \mathbf{h}_{E}^{-1}\left(\mathcal{G}_{E}^{*}\left(B z_{O}+\mathbf{h}_{E} \vec{N} \nabla_{x^{\prime}} q\right)\right)=\operatorname{g} \text { on } Q \\
\left.z_{O}\right|_{\Sigma}=0,\left.\quad q\right|_{\Sigma}=0,\left.\quad \partial_{\vec{n}} q\right|_{\Sigma}=0 .
\end{gathered}
$$

We need the following standard result on solvability of the system (4.65)-(4.67) equipped with the following initial conditions

$$
z_{O}(\hat{\tau}, \cdot)=z_{O}^{0}, \partial_{x_{0}} z_{O}(\hat{\tau}, \cdot)=z_{O}^{1}, q(\hat{\tau}, \cdot)=q^{0}, \partial_{x_{0}} q(\hat{\tau}, \cdot)=q^{1} .
$$


Proposition 4.1. Let $\hat{\tau} \in[0, T]$ be an arbitrary point. Suppose that $z_{O}^{0} \in \mathbf{L}_{O}^{2}(\Omega), z_{O}^{1} \in \mathbf{H}_{O}^{-1}(\Omega), q^{0} \in$ $H_{0}^{1}(\Omega), q^{1} \in L^{2}(\Omega),(h, \mathbf{g}) \in L^{2}\left(0, T ; H^{-2}(\Omega)\right) \times L^{2}\left(0, T ; H^{-1}(\Omega)\right)$. Then there exists a unique solution to the problem (4.65)-(4.68) which satisfies:

$$
\begin{aligned}
\|q\|_{L^{2}\left(0, T ; H^{1}(\Omega)\right)}+\left\|z_{O}\right\|_{\mathbf{L}_{O}^{2}(Q)} \leq & C\left(\|(h, \mathbf{g})\|_{L^{2}\left(0, T ; H^{-2}(\Omega)\right) \times L^{2}\left(0, T ; H^{-1}(\Omega)\right)}\right. \\
& \left.+\left\|\left(z_{O}^{0}, z_{O}^{1}\right)\right\|_{\mathbf{L}_{O}^{2}(\Omega) \times \mathbf{H}_{O}^{-1}(\Omega)}+\left\|\left(q^{0}, q^{1}\right)\right\|_{H^{1}(\Omega) \times L^{2}(\Omega)}\right) .
\end{aligned}
$$

The proof of this proposition can be easily done by transposition method.

Using the Carleman estimates obtained in Lemmata 4.3 and 4.5 we arrive to the following inequality:

Lemma 4.7. There exists a constant $C$ such that any solution $z_{O} \in \mathbf{L}_{O}^{2}(Q), q \in L^{2}\left(0, T ; H^{1}(\Omega)\right)$ to system (4.65)-(4.67) satisfies the estimate

$$
\left\|z_{O}\right\|_{\mathbf{L}_{O}^{2}(Q)}+\|q\|_{L^{2}\left(0, T ; H^{1}(\Omega)\right)} \leq C\left(\|(h, \mathbf{g})\|_{L^{2}\left(0, T ; H^{-2}(\Omega)\right) \times L^{2}\left(0, T ; H^{-1}(\Omega)\right)}+\left\|z_{O}\right\|_{\mathbf{L}_{O}^{2}\left(Q_{\omega}\right)}+\|q\|_{H^{1}\left(Q_{\omega}\right)}\right) .
$$

Proof. Denote

$$
\mathbf{f}=\mathbf{g}-B^{T} \mathbf{h}_{E}^{-1}\left(\mathcal{G}_{E}^{*}\left(B z_{O}+\mathbf{h}_{E} \vec{N} \nabla_{x^{\prime}} q\right)\right) .
$$

Using the new notation one can rewrite (4.66) as

$$
\mathbf{h}_{O} \mathbf{p}_{O} \partial_{x_{0}}^{2} z_{O}-12 \mathbf{h}_{O} \mathbf{D}_{O} L_{O}(D) z_{O}=\mathbf{f}
$$

Next we denote

$$
r=h+\operatorname{div}_{x^{\prime}} \vec{N}^{T}\left(\mathcal{G}_{E}^{*}\left(B z_{O}+\mathbf{h}_{E} \vec{N} \nabla_{x^{\prime}} q\right)\right) .
$$

Using this notation we rewrite (4.65) as

$$
m \partial_{x_{0}}^{2} q-\alpha \Delta_{x^{\prime}} \partial_{x_{0}}^{2} q+K \Delta_{x^{\prime}}^{2} q=r .
$$

Let $\chi=\chi\left(x_{0}\right)$ be a cut-off function such that

$$
\chi \in C_{0}^{\infty}[0, T],\left.\quad \chi\right|_{[\delta, T-\delta]} \equiv 1,
$$

where $\delta>0$ is sufficiently small such that

$$
\inf _{x \in \overline{\Omega \backslash \omega}} \psi\left(\tau, x^{\prime}\right)>\sup _{x \in([0, \delta] \cup[T-\delta, T]) \times \overline{\Omega \backslash \omega}} \psi(x) .
$$

Denote $\tilde{z}_{O}=\chi z_{O}, \tilde{q}=\chi q$. The functions $\tilde{z}_{O}, \tilde{q}$ satisfy the equations

$$
\begin{aligned}
\mathbf{h}_{O} \mathbf{p}_{O} \partial_{x_{0}}^{2} \tilde{z}_{O}-12 \mathbf{h}_{O} \mathbf{D}_{O} L_{O}(D) \tilde{z}_{O} & =\chi \mathbf{f}-\left[\chi, \mathbf{h}_{O} \mathbf{p}_{O} \partial_{x_{0}}^{2}\right] z_{O} \\
m \partial_{x_{0}}^{2} \tilde{q}-\alpha \Delta_{x^{\prime}} \partial_{x_{0}}^{2} \tilde{q}+K \Delta_{x^{\prime}}^{2} \tilde{q} & =\chi r+\left[\chi,-m \partial_{x_{0}}^{2}+\alpha \Delta_{x^{\prime}} \partial_{x_{0}}^{2}\right] q .
\end{aligned}
$$

Applying to the equation (4.72) the Carleman estimate (4.19) we obtain for $s \geq s_{0}(\tau)$ the following:

$$
\begin{aligned}
\left\|\tilde{z}_{O} \mathrm{e}^{s \varphi}\right\|_{\mathbf{L}_{O}^{2}(Q)} \leq & C_{1}\left(\left\|\mathbf{g} \mathrm{e}^{s \varphi}\right\|_{\mathbf{H}_{O}^{-1, s}(Q)}+\left\|q \mathrm{e}^{s \varphi}\right\|_{L^{2}(Q)}+\left\|\nabla q \mathrm{e}^{s \varphi}\right\|_{\mathbf{L}^{2}(Q)}\right. \\
& \left.+\left\|z_{O} \mathrm{e}^{s \varphi}\right\|_{\mathbf{L}_{O}^{2}\left(Q_{\omega}\right)}+\left\|\left(\left[\chi, \mathbf{h}_{O} \mathbf{p}_{O} \partial_{x_{0}}^{2}\right] z_{O}\right) \mathrm{e}^{s \varphi}\right\|_{\mathbf{H}_{O}^{-1, s}(Q)}\right) .
\end{aligned}
$$


Applying to (4.73) Carleman estimate (4.19) we obtain for $s \geq s_{0}(\tau)$ :

$$
\begin{aligned}
s\left\|\tilde{q} \mathrm{e}^{s \varphi}\right\|_{L^{2}(Q)}+\left\|\nabla \tilde{q} \mathrm{e}^{s \varphi}\right\|_{\mathbf{L}^{2}(Q)} \leq & C\left(\left\|\left(h \mathrm{e}^{s \varphi}, \mathbf{g e}^{s \varphi}\right)\right\|_{L^{2}\left(0, T ; H^{-2, s}(\Omega)\right) \times L^{2}\left(0, T ; H^{-1, s}(\Omega)\right)}\right. \\
& +s\left\|\tilde{q} \mathrm{e}^{s \varphi}\right\|_{L^{2}\left(Q_{\omega}\right)}+\left\|\nabla q \mathrm{e}^{s \varphi}\right\|_{\mathbf{L}^{2}\left(Q_{\omega}\right)}+\left\|z_{O} \mathrm{e}^{s \varphi}\right\|_{\mathbf{L}_{O}^{2}(Q)} \\
& \left.+\left\|\nabla \tilde{q} \mathrm{e}^{s \varphi}\right\|_{\mathbf{L}^{2}(Q)}+\left\|\left(\left[\chi,-m \partial_{x_{0}}^{2}+\alpha \Delta_{x^{\prime}} \partial_{x_{0}}^{2}\right] q\right) \mathrm{e}^{s \varphi}\right\|_{X^{*}}\right) .
\end{aligned}
$$

Combining this with the previous estimate gives $\forall s \geq s_{0}(\tau)$ :

$$
\begin{array}{r}
\left\|\tilde{z}_{O} \mathrm{e}^{s \varphi}\right\|_{\mathbf{L}_{O}^{2}(Q)}+s\left\|\tilde{q} \mathrm{e}^{s \varphi}\right\|_{L^{2}(Q)}+\left\|\nabla \tilde{q} \mathrm{e}^{s \varphi}\right\|_{\mathbf{L}^{2}(Q)} \leq C\left(\left\|z_{O} \mathrm{e}^{s \varphi}\right\|_{\mathbf{L}_{O}^{2}\left(Q_{\omega}\right)}+s\left\|\tilde{q} \mathrm{e}^{s \varphi}\right\|_{L^{2}\left(Q_{\omega}\right)}\right. \\
+\left\|\nabla q \mathrm{e}^{s \varphi}\right\|_{\mathbf{L}^{2}\left(Q_{\omega}\right)}+\left\|(h, \mathbf{g}) \mathrm{e}^{s \varphi}\right\|_{L^{2}\left(0, T ; H^{-2, s}(\Omega)\right) \times L^{2}\left(0, T ; H^{-1, s}(\Omega)\right)} \\
\left.+\left\|\left(\left[\chi, \mathbf{h}_{O} \mathbf{p}_{O} \partial_{x_{0}}^{2}\right] z_{O}\right) \mathrm{e}^{s \varphi}\right\|_{\mathbf{H}_{O}^{-1, s}(Q)}+\left\|\left(\left[\chi,-m \partial_{x_{0}}^{2} q+\alpha \Delta_{x^{\prime}} \partial_{x_{0}}^{2}\right] q\right) \mathrm{e}^{s \varphi}\right\|_{X^{*}}\right) .
\end{array}
$$

Thanks to Assumption A there exists $\tau \in[0, T]$ and $\varepsilon>0$ such that

$$
\varphi_{m}=\min _{x \in[-\varepsilon+\tau, \varepsilon+\tau] \times \overline{\Omega \backslash \omega}} \varphi(x)>\max _{x \in[0, \varepsilon] \cup[T-\varepsilon, T] \times \overline{\Omega \backslash \omega}} \varphi(x)=\varphi_{M} .
$$

We set

$$
E\left(x_{0}\right)=\left\|z_{O}\left(x_{0}, \cdot\right)\right\|_{\mathbf{L}_{O}^{2}(\Omega)}^{2}+\left\|\partial_{x_{0}} z_{O}\left(x_{0}, \cdot\right)\right\|_{\mathbf{H}_{O}^{-1}(\Omega)}^{2}+\left\|\partial_{x_{0}} q\left(x_{0}, \cdot\right)\right\|_{L^{2}(\Omega)}^{2}+\left\|\nabla_{x^{\prime}} q\left(x_{0}, \cdot\right)\right\|_{\mathbf{L}^{2}(\Omega)}^{2} .
$$

Applying Proposition 2.1, obviously we have

$$
\begin{array}{rl}
E(\tau) \mathrm{e}^{s \varphi_{m}} \leq C & C\left(\left\|\tilde{z}_{O} \mathrm{e}^{s \varphi}\right\|_{\mathbf{L}_{O}^{2}(Q)}^{2}+s^{\frac{1}{2}}\left\|\tilde{q} \mathrm{e}^{s \varphi}\right\|_{L^{2}(Q)}^{2}+\left\|\nabla \tilde{q} \mathrm{e}^{s \varphi}\right\|_{\mathbf{L}^{2}(Q)}^{2}+\|q\|_{H^{1}\left(Q_{\omega}\right)}^{2}\right) \\
& +\tilde{C}(s)\left(\|(h, \mathbf{g})\|_{L^{2}\left(0, T ; H^{-2, s}(\Omega)\right) \times L^{2}\left(0, T ; H^{-1, s}(\Omega)\right)}^{2}+\left\|z_{O}\right\|_{L^{2}\left(Q_{\omega}\right)}\right)
\end{array}
$$

and

$$
\left\|\left(\left[\chi, \mathbf{h}_{O} \mathbf{p}_{O} \partial_{x_{0}}^{2}\right] z_{O}\right) \mathrm{e}^{s \varphi}\right\|_{\mathbf{H}_{O}^{-1, s}(Q)}^{2}+\left\|\left(\left[\chi,-m \partial_{x_{0}}^{2}+\alpha \Delta_{x^{\prime}} \partial_{x_{0}}^{2}\right] q\right) \mathrm{e}^{s \varphi}\right\|_{X^{*}}^{2} \leq C E(\tau) \mathrm{e}^{s \varphi_{M}}
$$

Finally by (4.74), (4.76), (4.77) we have

$$
\begin{aligned}
E(\tau) \mathrm{e}^{s \varphi_{m}} \leq & C\left(E(\tau) \mathrm{e}^{s \varphi_{M}}+\left\|z_{O} \mathrm{e}^{s \varphi}\right\|_{\mathbf{L}_{O}^{2}\left(Q_{\omega}\right)}^{2}+s^{2}\left\|q \mathrm{e}^{s \varphi}\right\|_{L^{2}\left(Q_{\omega}\right)}^{2}+\left\|\nabla q \mathrm{e}^{s \varphi}\right\|_{\mathbf{L}^{2}\left(Q_{\omega}\right)}^{2}\right) \\
& +\tilde{C}(s)\|(h, \mathbf{g})\|_{L^{2}\left(0, T ; H^{-2, s}(\Omega)\right) \times L^{2}\left(0, T ; H^{-1, s}(\Omega)\right)}^{2} .
\end{aligned}
$$

Taking the value of the parameter $s$ sufficiently large by (4.75) from (4.78) we obtain (4.70). The proof of the Lemma is finished.

Proof of Theorem 3.1. This theorem follows from the observability estimate in Lemma 4.7 and the duality between controllability and observability (Hilbert's Uniqueness Method; $c f$. Lions [18]). One can alternatively derive the controls as solutions of a limiting extremal problem. We sketch this approach.

Let $\mathcal{P}(D)\left(w, v_{O}\right)=\left(\mathcal{P}_{1}(D)\left(w, v_{O}\right), \mathcal{P}_{2}(D)\left(w, v_{O}\right)\right)$ denote the expressions in $(3.1),(3.2)$. 
Without loss of generality we may assume the initial data $v_{0}, v_{1}, w_{0}, w_{1}$ are all zero. Consider the extremal problem

$$
\begin{array}{r}
\mathcal{F}\left(w, v_{O}, f_{3}, f_{O}\right)=\left\|\left(w, v_{O}\right)\right\|_{L^{2}\left(0, T ; H^{2}(\Omega) \times \mathbf{H}_{O}^{1}(\Omega)\right)}^{2}+\left\|f_{3}\right\|_{L^{2}\left(Q_{\omega}\right)}^{2} \\
+\left\|f_{O}\right\|_{\mathbf{L}^{2}\left(Q_{\omega}\right)}^{2}+\frac{1}{\varepsilon}\left\|\left(u_{3}, u_{O}\right)\right\|_{\mathbf{L}^{2}(Q)}^{2} \rightarrow \text { inf, } \\
\mathcal{P}(D)\left(w, v_{O}\right)-\left(\sum_{k=0}^{2} \partial_{x_{k}} f_{3, k}, f_{O}\right)+\left(q_{3}, q_{O}\right)=\left(u_{3}, u_{O}\right) \quad \text { in } Q, \\
\left.\left(w, v_{O}\right)\right|_{\Sigma}=0,\left.\partial_{\vec{n}} w\right|_{\Sigma}=0, \\
\left.\left(w, v_{O}\right)\right|_{x_{0}=0}=\left.\left(\partial_{x_{0}} w, \partial_{x_{0}} v_{O}\right)\right|_{x_{0}=0}=\left.\left(w, v_{O}\right)\right|_{x_{0}=T}=\left.\left(\partial_{x_{0}} w, \partial_{x_{0}} v_{O}\right)\right|_{x_{0}=T}=0 .
\end{array}
$$

Denote the solution to this problem by $\left(\hat{w}_{\varepsilon}, \hat{v}_{O, \varepsilon}, \hat{f}_{3, \varepsilon}, \hat{f}_{O, \varepsilon}, \hat{u}_{3, \varepsilon}, \hat{u}_{O, \varepsilon}\right)$. This solution exists and is unique. We set $p_{\varepsilon}=\left(p_{3, \varepsilon}, p_{O, \varepsilon}\right)=\frac{1}{\varepsilon}\left(\hat{u}_{3, \varepsilon}, \hat{u}_{O, \varepsilon}\right)$. Define the space of test functions

$$
\widetilde{\mathcal{D}}=\left\{\left(\delta_{3}, \delta_{O}\right) \in H^{2}(Q) \times \mathbf{H}^{1}(Q): \mathcal{P}(D)\left(\delta_{3}, \delta_{O}\right) \in L^{2}(Q),\left.\left(\delta_{3}, \delta_{O}\right)\right|_{\partial Q}=0,\left.\partial_{\vec{n}} \delta_{3}\right|_{\partial Q}=0\right\} .
$$

By Fermat's theorem,

$$
\left(\left(\hat{w}_{\varepsilon}, \hat{v}_{O, \varepsilon}\right),\left(\delta_{3}, \delta_{O}\right)\right)_{L^{2}\left(0, T ; H^{2}(\Omega) \times \mathbf{H}_{O}^{1}(\Omega)\right)}+\left(p_{\varepsilon}, \mathcal{P}(D)\left(\delta_{3}, \delta_{O}\right)\right)_{L^{2}(Q)}=0, \quad \forall\left(\delta_{3}, \delta_{O}\right) \in \widetilde{\mathcal{D}} .
$$

This implies

$$
\begin{aligned}
\mathcal{P}^{*}(D)\left(p_{3, \varepsilon}, p_{O, \varepsilon}\right)=-\left(\Delta_{x^{\prime}}^{2} \hat{w}_{\varepsilon}+\hat{w}_{\varepsilon},-\Delta_{x^{\prime}} \hat{v}_{O, \varepsilon}+\hat{v}_{O, \varepsilon}\right) \\
\\
\left.\left(p_{3, \varepsilon}, p_{O, \varepsilon}\right)\right|_{\Sigma}=0,\left.\quad \partial_{\vec{n}} p_{3, \varepsilon}\right|_{\Sigma}=0 .
\end{aligned}
$$

Note that $\left(p_{3, \varepsilon}, p_{O, \varepsilon}\right) \in L^{2}\left(0, T ; H^{2}(\Omega) \times \mathbf{H}_{O}^{1}(\Omega)\right)$.

Also

$$
\begin{aligned}
\partial_{x_{k}} \hat{u}_{3, \varepsilon}+\varepsilon \hat{f}_{3, k}=0 \quad k=0,1,2 & \text { in } Q_{\omega} \\
\hat{u}_{O, \varepsilon}+\varepsilon \hat{f}_{O, \varepsilon}=0 & \text { in } Q_{\omega} .
\end{aligned}
$$

Applying to (4.82), (4.83) observability estimate (4.69) we have

$$
\left\|p_{\varepsilon}\right\|_{L^{2}\left(0, T ; H^{1}(\Omega) \times \mathbf{L}_{O}^{2}(\Omega)\right)} \leq C\left(\left\|\left(\hat{w}, \hat{v}_{O}\right)\right\|_{L^{2}\left(0, T ; H^{2}(\Omega) \times \mathbf{H}_{O}^{1}(\Omega)\right)}+\left\|\hat{f}_{3}\right\|_{L^{2}\left(Q_{\omega}\right)}+\left\|\hat{f}_{O}\right\|_{\mathbf{L}^{2}\left(Q_{\omega}\right)}\right) .
$$

If we plug in $(4.81)$ instead of $\left(\delta_{3}, \delta_{O}\right)$, the pair $\left(\hat{w}_{,} \hat{v}_{O}\right)$, we have

$$
\mathcal{F}\left(\hat{w}_{\varepsilon}, \hat{v}_{O, \varepsilon}, \hat{f}_{3, \varepsilon}, \hat{f}_{O, \varepsilon}\right)=-\left(p_{\varepsilon},\left(q_{3}, q_{O}\right)\right)_{L^{2}(Q)} .
$$

By (4.86) we have

$$
\mathcal{F}\left(\hat{w}_{\varepsilon}, \hat{v}_{O, \varepsilon}, \hat{f}_{3, \varepsilon}, \hat{f}_{O, \varepsilon}\right) \leq C\left\|\left(q_{3}, q_{O}\right)\right\|_{L^{2}\left(0, T ; H^{-1}(\Omega) \times \mathbf{L}^{2}(\Omega)\right)}^{2} .
$$

Using (4.87) and the standard a priori estimates for the system (3.1)-(3.4) we obtain:

$$
\begin{array}{r}
\left\|\left(\hat{w}_{\varepsilon}, \hat{v}_{O, \varepsilon}\right)\right\|_{L^{2}\left(0, T ; H^{2}(\Omega) \times \mathbf{H}_{O}^{1}(\Omega)\right)}+\left\|\partial_{x_{0}} \hat{w}_{\varepsilon}\right\|_{L^{2}\left(0, T ; H_{0}^{1}(\Omega)\right)}+\left\|\hat{f}_{3, \varepsilon}\right\|_{L^{2}\left(Q_{\omega}\right)}+\left\|\hat{f}_{O, \varepsilon}\right\|_{\mathbf{L}^{2}\left(Q_{\omega}\right)} \\
\leq C\left\|\left(q_{3}, q_{O}\right)\right\|_{L^{2}\left(0, T ; H^{-1}(\Omega) \times \mathbf{L}^{2}(\Omega)\right)} .
\end{array}
$$

By (4.88) we can take a subsequence from $\left(\hat{w}_{\varepsilon}, \hat{v}_{O, \varepsilon}, \hat{f}_{3, \varepsilon}, \hat{f}_{O, \varepsilon}\right)$ which converges weakly in $L^{2}\left(0, T ; H^{2}(\Omega) \times\right.$ $\left.\mathbf{H}_{O}^{1}(\Omega)\right) \times \mathbf{L}^{2}\left(Q_{\omega}\right)$ to a function $\left(w, v_{O}, f_{3}, f_{O}\right)$. Passing to the limit in (4.80) we obtain that the element $\left(w, v_{O}, f_{3}, f_{O}\right)$ is a solution to our controllability problem. The proof of Theorem 3.1 is finished. 


\subsection{Simply supported boundary conditions}

In order to treat the boundary conditions (3.6) we consider the following problem

$$
\begin{gathered}
\mathcal{K}(D) v=m \partial_{x_{0}^{2}}^{2} v-\alpha \Delta_{x^{\prime}} \partial_{x_{0}^{2}}^{2} v+K \Delta_{x^{\prime}}^{2} v=f \text { in } Q \\
\left.v\right|_{\Sigma}=0,\left.\partial_{\vec{n}}^{2} v\right|_{\Sigma}=0, v(0, \cdot)=v(T, \cdot)=\partial_{x_{0}} v(0, \cdot)=\partial_{x_{0}} v(T, \cdot)=0 .
\end{gathered}
$$

Lemma 4.8. Let $\psi(x) \in C^{2}(\bar{Q})$ be a function pseudoconvex respect to the symbol $\xi_{0}^{2}-\frac{K}{\alpha}\left|\xi^{\prime}\right|^{2}$ such that $\frac{\partial \psi}{\partial \vec{n}}|\overline{(0, T) \times(\Gamma \backslash \partial \omega)}<0,| \nabla_{x^{\prime}} \psi \mid \neq 0$ on $[0, T] \times \overline{\Omega \backslash \omega}$, and let the function $\varphi$ given by formula (4.2). Suppose that $v \in L^{2}\left(0, T ; H^{2}(\Omega)\right)$ is a solution to problem (4.89), (4.90) with the right hand side $f=\tilde{f}+\sum_{j=0}^{2} \partial_{x_{i}} f_{i}$, with $\tilde{f} \in H^{-1}(Q), f_{i} \in L^{2}(Q)$. Then there exists $\tau_{0}>1$ such that for any $\tau>\tau_{0}$ there exists $s_{0}(\tau)>0$ such that $\forall s>s_{0}(\tau)$

$$
\left\|v \mathrm{e}^{s \varphi}\right\|_{H^{2, s}(Q)}^{2} \leq C_{1}\left(\left\|\tilde{f} \mathrm{e}^{s \varphi}\right\|_{H^{-1, s}(Q)}^{2}+\sum_{i=0}^{2}\left\|f_{i} \mathrm{e}^{s \varphi}\right\|_{L^{2}(Q)}^{2}+\int_{Q_{\omega}}\left(s^{4} v^{2}+s\left|\Delta_{x^{\prime}} v\right|^{2}\right) \mathrm{e}^{2 s \varphi} \mathrm{d} x\right)
$$

and $C_{1}(\tau) \rightarrow 0$ as $\tau \rightarrow+0$.

Proof. Using the Carleman estimate (4.3) we obtain $\forall s \geq s_{0}(\tau)$ the following

$$
\int_{Q} s\left|\Delta_{x^{\prime}} v\right|^{2} \mathrm{e}^{2 s \varphi} \mathrm{d} x \leq C_{2}\left(\left\|\tilde{f} \mathrm{e}^{s \varphi}\right\|_{H^{-1}(Q)}^{2}+\sum_{i=0}^{2}\left\|f_{i} \mathrm{e}^{s \varphi}\right\|_{L^{2}(Q)}^{2}+\int_{Q_{\omega}} s\left|\Delta_{x^{\prime}} v\right|^{2} \mathrm{e}^{2 s \varphi} \mathrm{d} x\right)
$$

and $C_{2}(\tau) \rightarrow 0$ as $\tau \rightarrow+0$. Thanks to (3.8), we have the Carleman estimate for elliptic equation (see $e . g$. [8]) which yields:

$$
\sum_{|\alpha|=0}^{2} \int_{Q} s^{3-2|\alpha|}\left|D^{\alpha} v\right|^{2} \mathrm{e}^{2 s \varphi} \mathrm{d} x \leq C_{3}\left(\int_{Q}\left|\Delta_{x^{\prime}} v\right|^{2} \mathrm{e}^{2 s \varphi} \mathrm{d} x+\int_{Q_{\omega}}|v|^{2} \mathrm{e}^{2 s \varphi} \mathrm{d} x\right) \quad \forall s \geq s_{0}(\tau)
$$

where $C_{3}(\tau) \rightarrow+0$ as $\tau \rightarrow+\infty$.

The estimates (4.92) and (4.93) imply (4.91). The proof of the lemma is finished.

For the simply supported boundary conditions, the analog of Lemma 4.7 is the following.

Lemma 4.9. Let $h=\sum_{i=0}^{2} \partial_{x_{i}} h_{i}, \mathbf{g} \in H_{O}^{-1}(Q), h_{i} \in L^{2}(Q), i=0,1,2$. There exists a constant $C$ such that any solution $z_{O} \in \mathbf{L}_{O}^{2}(Q), q \in L^{2}\left(0, T ; H^{2}(\Omega)\right)$ to system (4.65), (4.66), (3.6) satisfies the estimate

$$
\begin{aligned}
\left\|z_{O}\right\|_{\mathbf{L}_{O}^{2}(Q)}+\|q\|_{L^{2}\left(0, T ; H^{2}(\Omega)\right)} \leq & C\left(\sum_{i=0}^{2}\left\|h_{i}\right\|_{L^{2}(Q)}+\|\mathbf{g}\|_{\mathbf{H}_{O}^{-1}(Q)}\right. \\
& \left.+\left\|z_{O}\right\|_{\mathbf{L}_{O}^{2}\left(Q_{\omega}\right)}+\|q\|_{L^{2}\left(0, T ; H^{2}(\omega)\right)}\right) .
\end{aligned}
$$

The proof of the Lemma 4.9 is exactly the same as the proof of the Lemma 4.7. The only difference is that instead of the estimate (4.19) we use the estimate (4.91). Likewise, Theorem 3.2 follows as a consequence of Lemma 4.9. 


\section{Appendix}

The following proposition can be found in [16].

Proposition 5.1. Let $w \in C\left(0, T, H_{0}^{2}(\Omega)\right)$ be a solution to the initial value problem

$$
\mathcal{K}(D) w=f,\left.\quad w\right|_{\Sigma}=\left.\partial_{\vec{n}} w\right|_{\Sigma}=0, \quad w(0, \cdot)=w_{0}, \partial_{x_{0}} w(0, \cdot)=w_{1}
$$

where $f \in L^{2}\left(0, T ;\left(H^{1}(\Omega)\right)^{*}\right), w_{0} \in H_{0}^{2}(\Omega), w_{1} \in H_{0}^{1}(\Omega)$. Then $\partial_{\vec{n}}^{2} w \in L^{2}(\Sigma)$.

Proof of Lemma 4.6. For any $\varepsilon \in(0,1)$, we consider the following extremal problem

$$
\begin{gathered}
J_{\varepsilon}\left(z, v_{0}, v_{1}, v_{2}, v_{3}, w\right)=\frac{1}{2} \int_{Q}\left(\left|\nabla_{x^{\prime}}\left(z \mathrm{e}^{-s \varphi}\right)\right|^{2}+s^{2}|z|^{2} \mathrm{e}^{-2 s \varphi}\right) \mathrm{d} x \\
+\frac{1}{2} \int_{Q} m_{\varepsilon}\left(\left|v_{0}\right|^{2}+\left|v_{1}\right|^{2}+\left|v_{2}\right|^{2}+\frac{\left|v_{3}\right|^{2}}{s^{2}}\right) \mathrm{e}^{-2 s \varphi} \mathrm{d} x+\frac{1}{2 \varepsilon} \int_{Q}|w|^{2} \mathrm{~d} x \longrightarrow \inf , \\
\mathcal{K}(D) z=\sum_{k=0}^{2} \partial_{x_{0} x_{k}}^{2} v_{k}+v_{3}-\mathrm{e}^{s \varphi} \Delta_{x^{\prime}}\left(v \mathrm{e}^{s \varphi}\right)+s^{2} v \mathrm{e}^{2 s \varphi}+w \quad \text { in } Q, \\
\left.\partial_{\vec{n}} z\right|_{\Sigma}=\left.z\right|_{\Sigma}=0, \quad \partial_{x_{0}} z\left(0, x^{\prime}\right)=\partial_{x_{0}} z\left(T, x^{\prime}\right)=0,
\end{gathered}
$$

where the penalty function $m_{\varepsilon}$ satisfies $m_{\varepsilon} \in C^{2}(\bar{\Omega}), m_{\varepsilon}>0$ on $\bar{\Omega}$, with $m_{\varepsilon}\left(x^{\prime}\right)=1$ if $x^{\prime} \in \omega$ and $m_{\varepsilon}\left(x^{\prime}\right)=\frac{1}{\varepsilon}$ if $\operatorname{dist}(x, \omega) \geq \frac{-1}{\ln \varepsilon}$.

We understand the equalities (5.2)-(5.3) in the weak sense

$$
(z, \mathcal{K}(D) \delta)_{L^{2}(Q)}=\left(\nabla_{x^{\prime}}\left(v \mathrm{e}^{\varphi}\right), \nabla_{x^{\prime}}\left(\delta \mathrm{e}^{\varphi}\right)\right)_{L^{2}(Q)}+\int_{Q}\left(\left(s^{2} v \mathrm{e}^{2 s \varphi}+v_{3}\right) \delta+\sum_{k=0}^{2} v_{k} \partial_{x_{0} x_{k}}^{2} \delta\right) \mathrm{d} x
$$

for all $\delta \in H^{2}(Q)$ such that $\mathcal{K}(D) \delta \in L^{2}(Q)$, with $\partial_{x_{0}} \delta(0, \cdot)=\partial_{x_{0}} \delta(T, \cdot)=0,\left.\delta\right|_{\Sigma}=\left.\partial_{\vec{n}} \delta\right|_{\Sigma}=0$.

Denote by $\left(\widehat{z}_{\varepsilon}, \widehat{\mathbf{v}}_{\varepsilon}, \widehat{w}_{\varepsilon}\right)=\left(\widehat{z}_{\varepsilon}, \widehat{v}_{0, \varepsilon}, \widehat{v}_{1, \varepsilon}, \widehat{v}_{2, \varepsilon}, \widehat{v}_{3, \varepsilon}, \widehat{w}_{\varepsilon}\right)$ the solution to extremal problem (5.1)-(5.3). We have:

Proposition 5.2. Under the conditions of Lemma 4.6 for all $v \in L^{2}\left(0, T ; H_{0}^{2}(\Omega)\right)$, there exists a unique solution $\left(\widehat{z}_{\varepsilon}, \widehat{\mathbf{v}}_{\varepsilon}, \widehat{w}_{\varepsilon}\right) \in H^{2}(Q) \times \mathbf{H}^{1}(Q) \times H^{2}(Q)$ to problem (5.1)-(5.3). Moreover this solution satisfies the optimality system:

$$
\begin{aligned}
& p_{\varepsilon}(x)=\frac{\widehat{w}_{\varepsilon}(x)}{\varepsilon} \quad \text { in } Q, \\
& \mathcal{K}(D) p_{\varepsilon}-\mathrm{e}^{-s \varphi} \Delta_{x^{\prime}}\left(\widehat{z}_{\varepsilon} \mathrm{e}^{-s \varphi}\right)+s^{2} \mathrm{e}^{-2 s \varphi} \widehat{z}_{\varepsilon}=0 \quad \text { in } Q \text {, } \\
& \left.\partial_{\vec{n}} p_{\varepsilon}\right|_{\Sigma}=\left.p_{\varepsilon}\right|_{\Sigma}=\left.\partial_{\vec{n}} \widehat{z}_{\varepsilon}\right|_{\Sigma}=\left.\widehat{z}_{\varepsilon}\right|_{\Sigma}=0, \\
& \partial_{x_{0}} p_{\varepsilon}(0, \cdot)=\partial_{x_{0}} p_{\varepsilon}(T, \cdot)=\partial_{x_{0}} \widehat{z}_{\varepsilon}(0, \cdot)=\partial_{x_{0}} \widehat{z}_{\varepsilon}(T, \cdot)=0 \text {, } \\
& \mathcal{K}(D) \widehat{z}_{\varepsilon}=\sum_{k=0}^{2} \partial_{x_{0} x_{k}}^{2} \widehat{v}_{k, \varepsilon}+\widehat{v}_{3, \varepsilon}-\mathrm{e}^{s \varphi} \Delta_{x^{\prime}}\left(\mathrm{e}^{s \varphi} v\right)+s^{2} v \mathrm{e}^{2 s \varphi}+\widehat{w}_{\varepsilon} \quad \text { in } Q \\
& -\partial_{x_{0} x_{k}}^{2} p_{\varepsilon}+m_{\varepsilon} \widehat{v}_{k, \varepsilon} \mathrm{e}^{-2 s \varphi}=0, k=0,1,2 ; \quad \text { in } Q, \\
& p_{\varepsilon}-m_{\varepsilon} \frac{\widehat{v}_{3, \varepsilon}}{s^{2}} \mathrm{e}^{-2 s \varphi}=0 \quad \text { in } Q,
\end{aligned}
$$


and there exists $s_{0}>0$ such that the following estimate holds:

$$
\begin{array}{r}
\left\|\nabla_{x^{\prime}}\left(\widehat{z}_{\varepsilon} \mathrm{e}^{-s \varphi}\right)\right\|_{H^{1, s}(Q)}^{2}+\sum_{k=0}^{2}\left\|\widehat{v}_{k, \varepsilon} \mathrm{e}^{-s \varphi}\right\|_{H^{1, s}\left(Q_{\omega}\right)}^{2}+s^{2}\left\|\widehat{v}_{k, \varepsilon} \mathrm{e}^{-s \varphi}\right\|_{L^{2}\left(Q_{\omega}\right)}^{2} \\
+\left\|\widehat{v}_{3, \varepsilon} \mathrm{e}^{-s \varphi}\right\|_{L^{2}\left(Q_{\omega}\right)}^{2}+\sum_{k=0}^{3} \frac{1}{s^{2}}\left\|m_{\varepsilon} \hat{v}_{k, \varepsilon}\right\|_{L^{2}(Q)}^{2} \leq C_{2}\left\|v \mathrm{e}^{s \varphi}\right\|_{L^{2}\left(0, T ; H^{1, s}(\Omega)\right)}^{2} \quad \forall s \geq s_{0},
\end{array}
$$

where $C_{2}$ is independent of $s$.

Proof of Proposition 5.2. Since the functional $J_{\varepsilon}$ is strictly convex and the set of admissible elements is a linear space, problem $(5.1)-(5.3)$ has at most one solution. First let us prove that there exists a solution to (5.1)-(5.3): an element $(\widehat{z}, \widehat{\mathbf{v}}, \widehat{w})$ in the space $\mathbf{L}^{2}(Q)$. Obviously $\left(0,0,0, \mathrm{e}^{-s \varphi} \Delta_{x^{\prime}}\left(\mathrm{e}^{-s \varphi} v\right)-s^{2} v \mathrm{e}^{-2 s \varphi}\right)$ is an admissible element and so the set of an admissible elements is not empty. Hence there exists a minimizing sequence $\left\{\left(\widehat{z}_{j, \varepsilon}, \widehat{\mathbf{v}}_{j, \varepsilon}, \widehat{w}_{j, \varepsilon}\right)\right\}_{j=1}^{\infty}$ such that

$$
\left(\widehat{z}_{j, \varepsilon}, \widehat{\mathbf{v}}_{j, \varepsilon}, \widehat{w}_{j, \varepsilon}\right) \rightarrow\left(\widehat{z}_{\varepsilon}, \widehat{\mathbf{v}}_{\varepsilon}, \widehat{w}_{\varepsilon}\right) \quad \text { weakly in } \mathbf{L}^{2}(Q) .
$$

Passing to the limit in (5.4) and using (5.13), we obtain that $\left(\widehat{z}_{\varepsilon}, \widehat{\mathbf{v}}_{\varepsilon}, \widehat{w}_{\varepsilon}\right)$ is an admissible element. On the other hand, since the functional $J_{\varepsilon}$ is lower semi-continuous with respect to the weak convergence in $L^{2}\left(0, T ; H^{1}(\Omega)\right) \times$ $\mathbf{L}^{2}(Q)$, this element is a solution to problem (5.1)-(5.3).

In order to obtain optimality system (5.5)-(5.11), we introduce the function $\mathbf{q}(\delta), \delta=\left(\delta_{0}, \delta_{1}, \delta_{2}, \delta_{3}, \delta_{4}\right)$ by

$$
\mathbf{q}(\delta)=J_{\varepsilon}\left(\widehat{z}_{\varepsilon}+\delta_{0} d_{0}, \widehat{v}_{0, \varepsilon}+\delta_{1} d_{1}, \widehat{v}_{1, \varepsilon}+\delta_{2} d_{2}, \widehat{v}_{2, \varepsilon}+\delta_{3} d_{3}, \widehat{v}_{3, \varepsilon}+\delta_{4} d_{4}, r(\delta)\right),
$$

where $d_{0} \in H^{2}(Q)$ with $\mathcal{K}(D) d_{0} \in L^{2}(Q), d_{1}, d_{2}, d_{3} \in H^{2}(Q), d_{4} \in L^{2}(Q)$,

$$
r(\delta)=\mathcal{K}(D)\left(\widehat{z}_{\varepsilon}+\delta_{0} d_{0}\right)-\left(\sum_{k=0}^{2} \partial_{x_{0} x_{k}}^{2}\left(\widehat{v}_{k, \varepsilon}+\delta_{k+1} d_{k+1}\right)+\widehat{v}_{3, \varepsilon}+\delta_{4} d_{4}\right)+\mathrm{e}^{s \varphi} \Delta_{x^{\prime}}\left(\mathrm{e}^{s \varphi} v\right)-s^{2} v \mathrm{e}^{2 s \varphi}
$$

Obviously the function $\mathbf{q}$ attains the minimum in $\mathbb{R}^{3}$ at 0 . Thus $\nabla \mathbf{q}(0)=0$. Moreover the equalities $\partial_{\delta_{1}} \mathbf{q}(0)=$ $\partial_{\delta_{2}} \mathbf{q}(0)=\partial_{\delta_{3}} \mathbf{q}(0)=\partial_{\delta_{4}} \mathbf{q}(0)=0$ imply

$$
\begin{aligned}
-\frac{1}{\varepsilon} \int_{Q} \widehat{w}_{\varepsilon} \partial_{x_{0} x_{k}}^{2} d_{k+1} \mathrm{~d} x+\int_{Q} m_{\varepsilon} \widehat{v}_{k, \varepsilon} d_{k+1} \mathrm{e}^{-2 s \varphi} \mathrm{d} x & =0, & & k=0,1,2, \\
\forall d_{k+1} \in H^{2}(Q) \text { such that } d_{k+1}(0, \cdot)=d_{k+1}(T, \cdot)=0,\left.d_{k+1}\right|_{\Sigma}=\left.\partial_{\vec{n}} d_{k+1}\right|_{\Sigma} & =0, & & k=0,1,2, \\
\text { and } \partial_{x_{0}} d_{1}(0, \cdot)=\partial_{x_{0}} d_{1}(T, \cdot) & =0, & & \\
-\frac{1}{\varepsilon} \int_{Q} \widehat{w}_{\varepsilon} d_{4} \mathrm{~d} x+\int_{Q} m_{\varepsilon} \frac{\widehat{v}_{3, \varepsilon} d_{4}}{s^{2}} \mathrm{e}^{-2 s \varphi} \mathrm{d} x & =0, & & \forall d_{4} \in L^{2}(Q) .
\end{aligned}
$$

On the other hand, these equalities are equivalent to

$$
\begin{aligned}
& \frac{1}{\varepsilon} \partial_{x_{0} x_{k}}^{2} \widehat{w}_{\varepsilon}-m_{\varepsilon} \widehat{v}_{k, \varepsilon} \mathrm{e}^{-2 s \varphi}=0 \quad k=0,1,2 \quad \text { in } Q, \\
& \frac{\widehat{w}_{\varepsilon}}{\varepsilon}-m_{\varepsilon} \frac{\widehat{v}_{3, \varepsilon}}{s^{2}} \mathrm{e}^{-2 s \varphi}=0 \quad \text { in } Q .
\end{aligned}
$$

By the equality $\partial_{\delta_{0}} \mathbf{q}(0)=0$, we obtain

$$
\left(\frac{\widehat{w}_{\varepsilon}}{\varepsilon}, \mathcal{K}(D) d_{0}\right)_{L^{2}(Q)}+\int_{Q} s^{2} \widehat{z}_{\varepsilon} d_{0} \mathrm{e}^{-2 s \varphi} \mathrm{d} x+\left(\nabla_{x^{\prime}}\left(\hat{z}_{\varepsilon} \mathrm{e}^{-s \varphi}\right), \nabla_{x^{\prime}}\left(d_{0} \mathrm{e}^{-s \varphi}\right)\right)_{L^{2}(Q)}=0, \quad \forall d_{0} \in Y
$$


where

$$
\begin{aligned}
Y= & \left\{d_{0} \in H^{4}(Q) ; \mathcal{K}(D) d_{0} \in L^{2}(Q),\left.\partial_{\vec{n}} d_{0}\right|_{\Sigma}=\left.d_{0}\right|_{\Sigma}=0\right. \\
& \left.\partial_{x_{0}} d_{0}(0, \cdot)=\partial_{x_{0}} d_{0}(T, \cdot)=0\right\} .
\end{aligned}
$$

Since $\widehat{v}_{k, \varepsilon} \in L^{2}(Q), k=0,1,2,3$ we obtain immediately from (5.14) that $\nabla \partial_{x_{0}} \widehat{w}_{\varepsilon} \in L^{2}(Q)$. Since $d_{0}(0, \cdot)$ and $d_{0}(T, \cdot)$ can be chosen arbitrarily, it follows from (5.16) that

$$
\partial_{x_{0}} \widehat{w}_{\varepsilon}(0, \cdot)=\partial_{x_{0}} \widehat{w}_{\varepsilon}(T, \cdot)=\left.0 \quad \partial_{\vec{n}} \widehat{w}_{\varepsilon}\right|_{\Sigma}=\left.\widehat{w}_{\varepsilon}\right|_{\Sigma}=0 .
$$

Introducing the function $p_{\varepsilon}$ by formula (5.5), in terms of (5.14)-(5.16), we immediately obtain equalities (5.5)(5.11). Equations (5.5) implies $\partial_{x_{0}} \nabla p_{\varepsilon} \in L^{2}(Q)$. Let $\rho_{1}\left(x_{0}\right) \in C^{\infty}[0, T]$ be a nonnegative function which vanishes around $x_{0}=0$ and $\rho_{1}\left(x_{0}\right)>0$ is positive for $x_{0} \in[T / 2, T]$.

Let $\rho_{2}\left(x_{0}\right) \in C^{\infty}[0, T]$ be a nonnegative function which is equal zero around $x_{0}=T$ and $\rho_{2}\left(x_{0}\right)>0$ is positive for $x_{0} \in[0, T / 2]$. We set $p_{\varepsilon, i}=\rho_{i} p_{\varepsilon}$. For $i=1$ or $i=2$ this function solves the initial value problem

$$
\begin{gathered}
\mathcal{K}(D) p_{\varepsilon, i}=-\left[\rho_{i}, m \partial_{x_{0}}^{2}-\alpha \Delta_{x^{\prime}} \partial_{x_{0}}^{2}\right] p_{\varepsilon}+\rho_{i} \mathrm{e}^{-s \varphi} \Delta_{x^{\prime}}\left(\widehat{z}_{\varepsilon} \mathrm{e}^{-s \varphi}\right)-s^{2} \mathrm{e}^{-2 s \varphi} \rho_{i} \widehat{z}_{\varepsilon} \quad \text { in } Q, \\
\left.\partial_{\vec{n}} p_{\varepsilon, i}\right|_{\Sigma}=\left.p_{\varepsilon, i}\right|_{\Sigma}=0, \\
p_{\varepsilon, 1}(0, \cdot)=\partial_{x_{0}} p_{\varepsilon, 1}(0, \cdot)=0, \quad \text { or } \\
p_{\varepsilon, 2}(T, \cdot)=\partial_{x_{0}} p_{\varepsilon, 2}(T, \cdot)=0 .
\end{gathered}
$$

The solution to the problem (5.17)-(5.20) satisfies $p_{\varepsilon, i} \in C\left(0, T ; H_{0}^{2}(\Omega)\right) \cap C^{1}\left(0, T ; H_{0}^{1}(\Omega)\right)$ and is unique. Hence $p_{\varepsilon} \in C\left(0, T ; H_{0}^{2}(\Omega)\right)$. Since

$$
\mathrm{e}^{-s \varphi} \Delta_{x^{\prime}}\left(\widehat{z}_{\varepsilon} \mathrm{e}^{-s \varphi}\right)-s^{2} \mathrm{e}^{-2 s \varphi} \widehat{z}_{\varepsilon} \in L^{2}\left(0, T ; H^{-1, s}(\Omega)\right)
$$

Proposition 5.1 implies $\partial_{\vec{n}}^{2} p_{\varepsilon} \in L^{2}(\Sigma)$. This implies $\partial_{x_{0}}^{2} p_{\varepsilon} \in L^{2}(Q)$. Hence $p_{\varepsilon} \in H^{2}(Q)$.

Next we will show that $p_{\varepsilon} \in H^{3}(Q)$.

We extend $p_{\varepsilon}, \hat{z}_{\varepsilon}$ on the set $[-T, 2 T] \times \Omega$ by the formula: $p_{\varepsilon}\left(x_{0}, x^{\prime}\right)=p_{\varepsilon}\left(-x_{0}, x^{\prime}\right), \hat{z}_{\varepsilon}\left(x_{0}, x^{\prime}\right)=\hat{z}_{\varepsilon}\left(-x_{0}, x^{\prime}\right)$ for $x \in[-T, 0] \times \Omega$ and $p_{\varepsilon}\left(x_{0}, x^{\prime}\right)=p_{\varepsilon}\left(2 T-x_{0}, x^{\prime}\right), \hat{z}_{\varepsilon}\left(x_{0}, x^{\prime}\right)=\hat{z}_{\varepsilon}\left(2 T-x_{0}, x^{\prime}\right)$ for $\left(x_{0}, x^{\prime}\right) \in[T, 2 T] \times \Omega$. In the same way, we extend $-\mathrm{e}^{-s \varphi} \Delta_{x^{\prime}}\left(\widehat{z}_{\varepsilon} \mathrm{e}^{-s \varphi}\right)+\widehat{z}_{\varepsilon} \mathrm{e}^{-2 s \varphi}$ on the domain $[-T, 2 T] \times \Omega$ and denote the extended function by $\widetilde{\mathbf{f}}$. Since $\partial_{x_{0}} \varphi\left(T, x^{\prime}\right)<0$ for all $x^{\prime} \in \bar{\Omega}$ and $\partial_{x_{0}} \varphi\left(0, x^{\prime}\right)>0$ for all $x^{\prime} \in \bar{\Omega}$, there exists $\delta>0$ such that we can continue the function $\varphi$ on $[-\delta, T+\delta] \times \Omega$ up to a $C^{3}$-function such that $\partial_{x_{0}} \varphi(x)<0$ for all $x \in[T, T+\delta] \times \bar{\Omega}$ and $\partial_{x_{0}} \varphi(x)>0$ for all $x \in[-\delta, 0] \times \bar{\Omega}$. By (5.6), we have

$$
\mathcal{K}(D) p_{\varepsilon}=\widetilde{\mathbf{f}} \quad \text { in } \widetilde{Q} \equiv[-\delta, T+\delta] \times \Omega .
$$

Let $D_{h} f=\frac{f\left(x_{0}+h, x^{\prime}\right)-f(x)}{h}$ and $D_{\bar{h}} f=\frac{f(x)-f\left(x_{0}-h, x^{\prime}\right)}{h}$. For the function $D_{h} D_{\bar{h}} p_{\epsilon}$, we have

$$
\left.\partial_{x_{0}} D_{h} D_{\bar{h}} p_{\varepsilon}\right|_{x_{0}=0}=\left.\partial_{x_{0}} D_{h} D_{\bar{h}} p_{\varepsilon}\right|_{x_{0}=T}=0 .
$$

Note that $\mathcal{K}(D) D_{h} D_{\bar{h}} p_{\varepsilon}=D_{h} D_{\bar{h}} \widetilde{\mathbf{f}}$. Hence

$$
\left(\widehat{z}_{\varepsilon}, D_{h} D_{\bar{h}} \widetilde{\mathbf{f}}\right)_{L^{2}(Q)}=\sum_{k=0}^{2}\left(\widehat{v}_{k, \varepsilon}, \partial_{x_{0}} \partial_{x_{k}} D_{h} D_{\bar{h}} p_{\varepsilon}\right)_{L^{2}(Q)}+\left(\widehat{v}_{3, \varepsilon}-\mathrm{e}^{s \varphi} \Delta_{x^{\prime}}\left(\mathrm{e}^{s \varphi} v\right)+s^{2} v \mathrm{e}^{2 s \varphi}+\widehat{w}_{\varepsilon}, D_{h} D_{\bar{h}} p_{\varepsilon}\right)_{L^{2}(Q)} .
$$


Using (5.9), (5.10) and the definition of the function $\tilde{\mathbf{f}}$, we have

$$
\begin{array}{r}
\frac{1}{2}\left(D_{h} \nabla_{x^{\prime}}\left(\widehat{z}_{\varepsilon} \mathrm{e}^{-s \varphi}\right), D_{h} \nabla_{x^{\prime}}\left(\mathrm{e}^{-s \varphi} \widehat{z}_{\varepsilon}\right)\right)_{L^{2}(Q)}+\frac{1}{2}\left(D_{\bar{h}} \nabla_{x^{\prime}}\left(\widehat{z}_{\varepsilon} \mathrm{e}^{-s \varphi}\right), D_{\bar{h}} \nabla_{x^{\prime}}\left(\mathrm{e}^{-s \varphi} \widehat{z}_{\varepsilon}\right)\right)_{L^{2}(Q)} \\
+\frac{s^{2}}{2}\left(D_{h} \widehat{z}_{\varepsilon}, D_{h}\left(\mathrm{e}^{-2 s \varphi} \widehat{z}_{\varepsilon}\right)\right)_{L^{2}(Q)}+\frac{s^{2}}{2}\left(D_{\bar{h}} \widehat{z}_{\varepsilon}, D_{\bar{h}}\left(\mathrm{e}^{-2 s \varphi} \widehat{z}_{\varepsilon}\right)\right)_{L^{2}(Q)} \\
+\left(\widehat{z}_{\varepsilon},\left[D_{h} D_{\bar{h}}, \mathrm{e}^{-s \varphi}\right]\left(\Delta_{x^{\prime}}\left(\widehat{z}_{\varepsilon} \mathrm{e}^{-s \varphi}\right)\right)\right)_{L^{2}(Q)}+\sum_{k=0}^{2}\left(\frac{1}{2}\left(D_{h} \widehat{v}_{k, \varepsilon}, D_{h}\left(m_{\varepsilon} \mathrm{e}^{-2 s \varphi} \widehat{v}_{k, \varepsilon}\right)\right)_{L^{2}(Q)}\right. \\
\left.+\frac{1}{2}\left(D_{\bar{h}} \widehat{v}_{k, \varepsilon}, D_{\bar{h}}\left(m_{\varepsilon} \mathrm{e}^{-2 s \varphi} \widehat{v}_{k, \varepsilon}\right)\right)_{L^{2}(Q)}\right)+\frac{1}{2}\left(D_{h} \widehat{v}_{3, \varepsilon}, D_{h}\left(s^{-2} m_{\varepsilon} \mathrm{e}^{-2 s \varphi} \widehat{v}_{3, \varepsilon}\right)\right)_{L^{2}(Q)} \\
+\frac{1}{2}\left(D_{\bar{h}} \widehat{r}_{3, \varepsilon}, D_{\bar{h}}\left(s^{-2} m_{\varepsilon} \mathrm{e}^{-2 s \varphi} \widehat{v}_{3, \varepsilon}\right)\right)_{L^{2}(Q)}+\frac{1}{2 \varepsilon}\left(D_{h} w_{\varepsilon}, D_{h} w_{\varepsilon}\right)_{L^{2}(Q)}+\frac{1}{2 \varepsilon}\left(D_{\bar{h}} w_{\varepsilon}, D_{\bar{h}} w_{\varepsilon}\right)_{L^{2}(Q)} \\
=\left(-\mathrm{e}^{s \varphi} \Delta_{x^{\prime}}\left(\mathrm{e}^{s \varphi} v\right)+s^{2} v \mathrm{e}^{2 s \varphi}, D_{h} D_{\bar{h}} p_{\varepsilon}\right)_{L^{2}(Q) .} .
\end{array}
$$

Hence

$$
\begin{array}{r}
\left\|D_{h} \widehat{z}_{\varepsilon}\right\|_{L^{2}\left(0, T ; H^{1}(\Omega)\right)}+\sum_{k=0}^{3}\left\|D_{h} \widehat{v}_{k, \varepsilon}\right\|_{L^{2}(Q)}+\left\|D_{\bar{h}} \widehat{z}_{\mathcal{\varepsilon}}\right\|_{L^{2}\left(0, T ; H^{1}(\Omega)\right)}+\sum_{k=0}^{3}\left\|D_{\bar{h}} \widehat{v}_{k, \varepsilon}\right\|_{L^{2}(Q)} \\
\leq C_{2}^{\prime}\left(\|v\|_{L^{2}\left(0, T ; H^{1}(\Omega)\right)}+\left\|\left(\widehat{z}_{\varepsilon}, \widehat{\mathbf{v}}_{\varepsilon}\right)\right\|_{L^{2}\left(0, T ; H^{1}(\Omega)\right) \times \mathbf{L}^{2}(Q)}\right),
\end{array}
$$

where the constant $C_{2}^{\prime}>0$ is independent of $h$. Therefore

$$
\left(\partial_{x_{0}} \nabla_{x^{\prime}} \widehat{z}_{\varepsilon}, \partial_{x_{0}} \widehat{\mathbf{v}}_{\varepsilon}\right) \in \mathbf{L}^{2}(Q) .
$$

Therefore the right hand side of equation (5.9) belongs to $L^{2}\left(0, T ; H^{-1}(\Omega)\right)$. Hence $\widehat{z}_{\varepsilon} \in H^{2}(Q)$. From (5.26) and equation (5.10) we see that $\partial_{x_{0}} p_{\varepsilon} \in H^{2}(Q)$. The function $\tilde{p}_{\varepsilon, i}=\rho_{i} \partial_{x_{0}} p_{\varepsilon}$

$$
\begin{gathered}
\mathcal{K}(D) \tilde{p}_{\varepsilon, i}=-\left[\rho_{i}, m \partial_{x_{0}}^{2}-\alpha \Delta_{x^{\prime}} \partial_{x_{0}}^{2}\right] \partial_{x_{0}} p_{\varepsilon}+\rho_{i} \partial_{x_{0}}\left(\mathrm{e}^{-s \varphi} \Delta_{x^{\prime}}\left(\widehat{z}_{\varepsilon} \mathrm{e}^{-s \varphi}\right)\right) \\
-s^{2} \rho_{i} \partial_{x_{0}}\left(\widehat{z}_{\varepsilon} \mathrm{e}^{-2 s \varphi}\right) \\
\left.\partial_{\vec{n}} \tilde{p}_{\varepsilon, i}\right|_{\Sigma}=\left.\tilde{p}_{\varepsilon, i}\right|_{\Sigma}=0, \\
\tilde{p}_{\varepsilon, 1}(0, \cdot)=\partial_{x_{0}} \tilde{p}_{\varepsilon, 1}(0, \cdot)=0, \quad \text { in } Q, \tilde{p}_{\varepsilon, 2}(T, \cdot)=\partial_{x_{0}} \tilde{p}_{\varepsilon, 2}(T, \cdot)=0 .
\end{gathered}
$$

The solution to the problem (5.27)-(5.29) satisfies $\tilde{p}_{\varepsilon, i} \in C\left(0, T ; H_{0}^{2}(\Omega)\right) \cap C^{1}\left(0, T ; H_{0}^{1}(\Omega)\right)$ and is unique. Hence $\partial_{x_{0}}^{2} p_{\varepsilon} \in C\left(0, T ; H_{0}^{1}(\Omega)\right)$. Since

$$
\mathrm{e}^{-s \varphi} \Delta_{x^{\prime}}\left(\widehat{z}_{\varepsilon} \mathrm{e}^{-s \varphi}\right)-s^{2} \mathrm{e}^{-2 s \varphi} \widehat{z}_{\varepsilon} \in L^{2}\left(0, T ; H^{-1}(\Omega)\right)
$$

Proposition 5.1 implies $\partial_{\vec{n}}^{2} \partial_{x_{0}} p_{\varepsilon} \in L^{2}(\Sigma)$. This implies $\partial_{x_{0}}^{3} p_{\varepsilon} \in L^{2}(Q)$. Hence $\partial_{x_{0}} p_{\varepsilon} \in H^{2}(Q)$. Finally solving the elliptic problem

$$
\frac{K}{\alpha} \Delta_{x^{\prime}}^{2} p_{\epsilon}=\Delta_{x^{\prime}} \partial_{x_{0}}^{2} p_{\varepsilon}-\frac{m}{\alpha} \partial_{x_{0}}^{2} p_{\varepsilon}+\frac{1}{\alpha}\left(\mathrm{e}^{-s \varphi} \Delta_{x^{\prime}}\left(\widehat{z}_{\varepsilon} \mathrm{e}^{-s \varphi}\right)-s^{2} \widehat{z}_{\varepsilon} \mathrm{e}^{-2 s \varphi}\right) ;\left.\quad p_{\varepsilon}\right|_{\Sigma}=\left.\partial_{\vec{n}} p_{\varepsilon}\right|_{\Sigma}=0
$$

we obtain $p_{\varepsilon} \in L^{2}\left(0, T ; H^{3}(\Omega)\right)$. Hence $p_{\varepsilon} \in H^{3}(Q)$.

Note that assumption (3.13) for the function $\varphi$ holds true if we replace the domains $Q, Q_{\omega}$ by $\widetilde{Q},[-\delta, T+\delta] \times \omega$ respectively. 
Let $\chi_{1} \in C_{0}^{\infty}(-\delta, T+\delta)$ be a cut-off function such that $\left.\chi_{1}\right|_{\left[-\frac{\delta}{2}, T+\frac{\delta}{2}\right]}=1$. Then

$$
\mathcal{K}(D)\left(\chi_{1} p_{\varepsilon}\right)=\chi_{1} \widetilde{\mathbf{f}}-\left[\chi_{1}, K\right] p_{\varepsilon} \quad \text { in }[-T, 2 T] \times \Omega,\left.\quad \chi_{1} p_{\varepsilon}\right|_{\Sigma}=\left.\partial_{\vec{n}} \chi_{1} p_{\varepsilon}\right|_{\Sigma}=0,
$$

where $\operatorname{supp}\left[\chi_{1}, \mathcal{K}\right] p_{\varepsilon} \subset\left(\left[T+\frac{\delta}{2}, T+\delta\right] \times \bar{\Omega}\right) \cup\left(\left[-\delta,-\frac{\delta}{2}\right] \times \bar{\Omega}\right)$. We will apply Carleman estimate (4.20) to equation (5.30).

For this, we observe that

$$
\begin{gathered}
\left\|\widetilde{\mathbf{f}}^{s \varphi}\right\|_{L^{2}\left(-\delta, T+\delta ;\left(H^{1, s}(\Omega)\right)^{*}\right)} \leq C_{3}\left\|\widehat{z}_{\varepsilon} \mathrm{e}^{-s \varphi}\right\|_{L^{2}\left(0, T ; H^{1, s}(\Omega)\right)}, \\
\left\|\left(\left[\chi_{1}, \mathcal{K}\right] p_{\varepsilon}\right) \mathrm{e}^{s \varphi}\right\|_{L^{2}\left(-\delta, T+\delta ;\left(H^{1, s}(\Omega)\right)^{*}\right)} \leq \frac{C_{4}}{s}\left\|p_{\varepsilon} \mathrm{e}^{s \varphi}\right\|_{H^{2, s}(Q)} .
\end{gathered}
$$

Next we prove that in the right hand side of (4.20) we can replace the second term $Q_{\omega}$ by the following integral

$$
\int_{Q_{\omega}}\left(\left|\partial_{x_{0}} \nabla v\right|^{2}+s^{2}|\nabla v|^{2}+s^{4}|v|^{2}\right) \mathrm{e}^{2 s \varphi} \mathrm{d} x
$$

Note that thanks to the choice of the extension of the function $\varphi$, we have

$$
\begin{gathered}
\int_{(-\delta, T+\delta) \times \omega}\left(\sum_{k=0}^{2}\left|\partial_{x_{0}} \partial_{x_{k}}\left(\chi_{1} p_{\varepsilon}\right)\right|^{2}+s^{2}\left|\nabla\left(\chi_{1} p_{\varepsilon}\right)\right|^{2}+s^{4}\left|\chi_{1} p_{\varepsilon}\right|^{2}\right) \mathrm{e}^{2 s \varphi} \mathrm{d} x \\
\leq C_{5} \int_{Q_{\omega}}\left(\sum_{k=0}^{2}\left|\partial_{x_{0}} \partial_{x_{k}} p_{\varepsilon}\right|^{2}+s^{2}\left|\nabla p_{\varepsilon}\right|^{2}+s^{4}\left|p_{\varepsilon}\right|^{2}\right) \mathrm{e}^{2 s \varphi} \mathrm{d} x
\end{gathered}
$$

In fact, let us denote the left and the right hand sides of (5.33) respectively by $I_{1}$ and $I_{2}$. For convenience, let us denote

$$
\Phi\left(p_{\varepsilon}\right)=\left(\sum_{k=0}^{2}\left|\partial_{x_{0}} \partial_{x_{k}} p_{\varepsilon}\right|^{2}+s^{2}\left|\nabla p_{\varepsilon}\right|^{2}+s^{4}\left|p_{\varepsilon}\right|^{2}\right),
$$

so that $I_{2}=C_{5} \int_{Q_{\omega}} \Phi\left(p_{\varepsilon}\right) \mathrm{e}^{2 s \varphi} \mathrm{d} x$. First, it is easy to see that

$$
I_{1} \leq C_{5}^{\prime} \int_{(-\delta, T+\delta) \times \omega} \Phi\left(p_{\varepsilon}\right) \mathrm{e}^{2 s \varphi} \mathrm{d} x .
$$

On the other hand, since $p_{\varepsilon}\left(x_{0}, x^{\prime}\right)=p_{\varepsilon}\left(-x_{0}, x^{\prime}\right),-\delta \leq x^{\prime} \leq 0$ by the extension, we have

$$
\int_{-\delta}^{0} \int_{\omega} \Phi\left(p_{\varepsilon}\right) \mathrm{e}^{2 s \varphi\left(x_{0}, x^{\prime}\right)} \mathrm{d} x_{0} \mathrm{~d} x^{\prime}=\int_{0}^{\delta} \int_{\omega} \Phi\left(p_{\varepsilon}\right) \mathrm{e}^{2 s \varphi\left(-x_{0}, x^{\prime}\right)} \mathrm{d} x_{0} \mathrm{~d} x^{\prime} .
$$

By (3.13), we have $\partial_{x_{0}} \varphi\left(0, x^{\prime}\right)>0$. Therefore, for all sufficiently small $\delta>0$, we obtain $\partial_{x_{0}} \varphi(x)>0$ for all $x_{0} \in[-\delta, \delta]$. This implies $\mathrm{e}^{2 s \varphi\left(-x_{0}, x^{\prime}\right)} \leq \mathrm{e}^{2 s \varphi\left(x_{0}, x^{\prime}\right)}$ for $0<x_{0}<\delta$. Hence

$$
\int_{0}^{\delta} \int_{\omega} \Phi\left(p_{\varepsilon}\right) \mathrm{e}^{2 s \varphi\left(-x_{0}, x^{\prime}\right)} \mathrm{d} x_{0} \mathrm{~d} x^{\prime} \leq \int_{0}^{\delta} \int_{\omega} \Phi\left(p_{\varepsilon}\right) \mathrm{e}^{2 s \varphi\left(x_{0}, x^{\prime}\right)} \mathrm{d} x_{0} \mathrm{~d} x^{\prime} \leq I_{2}
$$

We can similarly estimate $\int_{T}^{T+\delta} \int_{\omega} \Phi\left(p_{\varepsilon}\right) \mathrm{e}^{2 s \varphi} \mathrm{d} x_{0} \mathrm{~d} x^{\prime}$. Thus the verification of (5.33) is complete.

Using equations (5.9), (5.10), (5.11) and estimate (5.33), (4.20) we obtain

$$
\left\|p_{\varepsilon} \mathrm{e}^{s \varphi}\right\|_{H^{2, s}(Q)}^{2} \leq C_{6} J\left(\widehat{z}_{\varepsilon}, \widehat{\mathbf{v}}_{\varepsilon}\right) \quad \forall s \geq s_{0}
$$


and

$$
\left\|\partial_{x_{0}} p_{\varepsilon} \mathrm{e}^{s \varphi}\right\|_{H^{2, s}(Q)}^{2} \leq C_{6} J\left(\partial_{x_{0}} \widehat{z}_{\varepsilon}, \partial_{x_{0}} \widehat{\mathbf{v}}_{\varepsilon}\right) \quad \forall s \geq s_{0} .
$$

Note that $p_{\varepsilon}$ is the eligible test function for (5.4)

$$
\begin{gathered}
\left(\sum_{k=1}^{2} \widehat{v}_{k, \varepsilon}, \partial_{x_{0} x_{k}}^{2} p_{\varepsilon}\right)_{L^{2}(Q)}+\left(\nabla_{x^{\prime}}\left(v \mathrm{e}^{s \varphi}\right), \nabla_{x^{\prime}}\left(p_{\varepsilon} \mathrm{e}^{s \varphi}\right)\right)_{\mathbf{L}^{2}(Q)}+\left(\widehat{v}_{3, \varepsilon}+s^{2} v \mathrm{e}^{2 s \varphi}+\widehat{w}_{\varepsilon}, p_{\varepsilon}\right)_{L^{2}(Q)} \\
=\left(\widehat{z}_{\varepsilon}, \mathcal{K}(D) p_{\varepsilon}\right)_{L^{2}(Q)}=-\left\|\widehat{z}_{\varepsilon} \mathrm{e}^{-s \varphi}\right\|_{L^{2}\left(0, T ; H^{1, s}(\Omega)\right)}^{2} .
\end{gathered}
$$

Then we transform the left hand side of this inequality using (5.9), (5.10). We obtain

$$
2 J_{\varepsilon}\left(\widehat{z}_{\varepsilon}, \widehat{\mathbf{v}}_{\varepsilon}, \widehat{w}_{\varepsilon}\right)=-\int_{Q}\left(\left(\nabla_{x^{\prime}}\left(v \mathrm{e}^{s \varphi}\right), \nabla_{x^{\prime}}\left(\mathrm{e}^{s \varphi} p_{\varepsilon}\right)\right)+s^{2} v \mathrm{e}^{2 s \varphi} p_{\varepsilon}\right) \mathrm{d} x .
$$

By (5.34), we obtain from this inequality that

$$
s J_{\varepsilon}\left(\widehat{z}_{\varepsilon}, \widehat{\mathbf{v}}_{\varepsilon}, \widehat{w}_{\varepsilon}\right) \leq C_{7}\left\|v \mathrm{e}^{s \varphi}\right\|_{L^{2}\left(0, T ; H^{1, s}(\Omega)\right)} J\left(\widehat{z}_{\varepsilon}, \widehat{\mathbf{v}}_{\varepsilon}\right)^{\frac{1}{2}} .
$$

Passing to the limit in (5.24) as $h \rightarrow 0$ we have

$$
\begin{aligned}
2 J_{\varepsilon}\left(\partial_{x_{0}} \widehat{z}_{\varepsilon}, \partial_{x_{0}} \widehat{\mathbf{v}}_{\varepsilon}, \partial_{x_{0}} \widehat{w}_{\varepsilon}\right) & =\int_{Q}\left\{\left(-\mathrm{e}^{s \varphi} \Delta_{x^{\prime}}\left(v \mathrm{e}^{s \varphi}\right)+s^{2} v \mathrm{e}^{2 s \varphi}, \partial_{x_{0}^{2}}^{2} p_{\varepsilon}\right)\right. \\
& \left.+2 s \partial_{x_{0}} \varphi\left(\partial_{x_{0}} \widehat{z}_{\varepsilon}, \widehat{z}_{\varepsilon}\right) \mathrm{e}^{-2 s \varphi}+2 s\left(\partial_{x_{0}} \widehat{z}_{\varepsilon}\right) m_{\varepsilon} \sum_{k=0}^{2}\left(\partial_{x_{0}} \widehat{v}_{k, \varepsilon}, \widehat{v}_{k, \varepsilon}\right) \mathrm{e}^{-2 s \varphi}+\frac{2 m_{\varepsilon}}{s} \partial_{x_{0}} \varphi\left(\partial_{x_{0}} \widehat{v}_{3, \varepsilon}, \widehat{v}_{3, \varepsilon}\right) \mathrm{e}^{-2 s \varphi}\right\} \mathrm{d} x
\end{aligned}
$$

This equality and (5.35), (5.36) implies

$$
J_{\varepsilon}\left(\partial_{x_{0}} \widehat{z}_{\varepsilon}, \partial_{x_{0}} \widehat{\mathbf{v}}_{\varepsilon}, \partial_{x_{0}} \widehat{w}_{\varepsilon}\right) \leq C_{8}\left\|v \mathrm{e}^{s \varphi}\right\|_{L^{2}\left(0, T ; H^{1, s}(\Omega)\right)} J\left(\partial_{x_{0}} \widehat{z}_{\varepsilon}, \partial_{x_{0}} \widehat{\mathbf{v}}_{\varepsilon}\right)^{\frac{1}{2}}
$$

Taking the scalar product of (5.9) with $\widehat{z}_{\varepsilon} \mathrm{e}^{-2 s \varphi}$ in $L^{2}(Q)$, we obtain

$$
\begin{array}{r}
\int_{Q}\left(m\left(\left(\partial_{x_{0}}+s \varphi_{x_{0}}\right)\left(\hat{z}_{\varepsilon} \mathrm{e}^{-s \varphi}\right)\right)^{2}+\sum_{k=1}^{2} \alpha\left(\left(\partial_{x_{k}}+s \varphi_{x_{k}}\right)\left(\partial_{x_{0}}+s \varphi_{x_{0}}\right)\left(\hat{z}_{\varepsilon} \mathrm{e}^{-s \varphi}\right)\right)^{2}\right) \mathrm{d} x \\
-K \int_{Q} \sum_{k=1}^{2}\left(\left(\partial_{x_{k}}+s \varphi_{x_{k}}\right)^{2}\left(\hat{z}_{\varepsilon} \mathrm{e}^{-s \varphi}\right)\right)^{2} \mathrm{~d} x \\
=\int_{Q}\left(\sum_{k=0}^{2} \widehat{v}_{k, \varepsilon} \partial_{x_{0}} \partial_{x_{k}}\left(\widehat{z}_{\varepsilon} \mathrm{e}^{-2 s \varphi}\right)+\left(\widehat{v}_{3, \varepsilon}-\mathrm{e}^{s \varphi} \Delta_{x^{\prime}}\left(\mathrm{e}^{s \varphi} v\right)+s^{2} v \mathrm{e}^{2 s \varphi}+\widehat{w}_{\varepsilon}\right) \widehat{z}_{\varepsilon} \mathrm{e}^{-2 s \varphi}\right) \mathrm{d} x .
\end{array}
$$

From this equality and (5.37) we immediately obtain (5.12). The proof of Proposition 5.2 is complete.

Now we finish the proof of Lemma 4.6. Obviously $\widehat{w}_{\varepsilon} \rightarrow 0$ in $L^{2}(Q)$ and $\widehat{\mathbf{v}}_{\varepsilon_{k}} \rightarrow 0$ in $\mathbf{L}^{2}\left(Q \backslash Q_{\omega}\right)$ as $\varepsilon \rightarrow+0$. In terms of (5.37), from the sequence $\left\{\left(\widehat{z}_{\varepsilon}, \widehat{\mathbf{v}}_{\varepsilon}, p_{\varepsilon}\right)\right\}$, one can extract a subsequence $\left\{\left(\widehat{z}_{\varepsilon_{k}}, \widehat{\mathbf{v}}_{\varepsilon_{k}}, p_{\varepsilon_{k}}\right)\right\}$ such that

$$
\begin{gathered}
\left(\widehat{z}_{\varepsilon_{k}}, \widehat{\mathbf{v}}_{\varepsilon_{k}}, p_{\varepsilon_{k}}\right) \rightarrow(\widehat{z}, \widehat{\mathbf{v}}, p) \\
\text { weakly in } H_{0}^{2}(Q) \times \mathbf{H}^{1}(Q) \times \mathbf{L}^{2}(Q) .
\end{gathered}
$$


Thanks to (5.38), we can pass to the limit in (5.6)-(5.12), so that the element $\left(\widehat{z}, \widehat{\mathbf{v}}_{0}, p\right)$ satisfies the equations

$$
\begin{array}{rr}
\mathcal{K}(D) p-\mathrm{e}^{-s \varphi} \Delta_{x^{\prime}}\left(\mathrm{e}^{-s \varphi} \widehat{z}\right)+s^{2} \mathrm{e}^{-2 s \varphi} \widehat{z}=0 & \text { in } Q, \\
\left.\partial_{\vec{n}} p\right|_{\Sigma}=\left.p\right|_{\Sigma}=\left.\widehat{z}\right|_{\Sigma}=\left.\partial_{\vec{n}} \widehat{z}\right|_{\Sigma}=0, & \\
\partial_{x_{0}} p(0, \cdot)=\partial_{x_{0}} p(T, \cdot)=\partial_{x_{0}} \widehat{z}(0, \cdot)=\partial_{x_{0}} \widehat{z}(T, \cdot)=0, & \\
\mathcal{K}(D) \widehat{z}=\sum_{k=0}^{2} \partial_{x_{0} x_{k}}^{2} \widehat{v}_{k}+\widehat{v}_{3}-\mathrm{e}^{s \varphi} \Delta_{x^{\prime}}\left(\mathrm{e}^{s \varphi} v\right)+s^{2} v e^{2 s \varphi} & \text { in } Q, \\
\partial_{x_{0} x_{k}}^{2} p+\widehat{v}_{k} \mathrm{e}^{-2 s \varphi}=0, k=0,1,2 \quad p-\frac{\widehat{v}_{3}}{s^{2}} \mathrm{e}^{-2 s \varphi}=0 & \text { in } Q,
\end{array}
$$

where $\operatorname{supp} \widehat{v}_{j} \subset \bar{Q}_{\omega}, \quad j=0,1,2,3,\left.\quad \widehat{v}_{k} n_{k}\right|_{\partial \omega \backslash \Gamma}=0 k=1,2$.

Estimate (4.64) follows from (5.13). Finally we note that $J_{\varepsilon}\left(\widehat{z}_{\varepsilon}, \widehat{\mathbf{v}}_{\varepsilon}, \widehat{w}_{\varepsilon}\right) \leq J(z, \mathbf{v})$ for all $\varepsilon \in(0,1)$. Hence $J(\widehat{z}, \widehat{\mathbf{v}}) \leq J(z, \mathbf{v})$, the element $(\widehat{z}, \widehat{\mathbf{v}})$ is a solution to extremal problem (4.55)-(4.57). Since a solution to this problem is unique, we have $\left(\widehat{z}, \widehat{\mathbf{v}}_{0}\right)=\left(z, v_{0}, v_{1}, v_{2}, v_{3}\right)$. The proof of Lemma 4.6 is complete.

\section{REFERENCES}

[1] R. Dautray and J.-L. Lions (with collaboration of M. Artola, M. Cessenat, H. Lanchon), Mathematical Analysis and Numerical Methods for Science and Technology, Volume 5: Evolution Problems I. Springer-Verlag (1992).

[2] R.A. DiTaranto, Theory of vibratory bending for elastic and viscoelastic layered finite-length beams. J. Appl. Mech. 32 (1965) 881-886.

[3] S.W. Hansen, Several related models for multilayer sandwich plates. Math. Models Methods Appl. Sci. 14 (2004) $1103-1132$.

[4] S.W. Hansen, Semigroup well-posedness of a multilayer Mead-Markus plate with shear damping, in Control and Boundary Analysis, Lect. Not. Pure Appl. Math. 240, Chapman \& Hall/CRC, Boca Raton (2005) 243-256.

[5] S.W. Hansen and R. Rajaram, Riesz basis property and related results for a Rao-Nakra sandwich beam. Discrete Contin. Dynam. Syst. Suppl. (2005) 365-375.

[6] L. Hörmander, Linear Partial Differential Equations. Springer-Verlag, Berlin (1963).

[7] O.Y. Imanuvilov, On Carleman estimates for hyperbolic equations. Asymptotic Anal. 32 (2002) 185-220.

[8] O.Y. Imanuvilov and J.P. Puel, Global Carleman estimates for weak solutions of elliptic nonhomogeneous Dirichlet problems. Int. Math. Res. Not. 16 (2003) 883-913.

[9] O.Y. Imanuvilov and M. Yamamoto, Carleman estimates and the non-stationary Lamé system and the application to an inverse problem. ESIAM: COCV 11 (2005) 1-56.

[10] O.Y. Imanuvilov and M. Yamamoto, Carleman estimates for the three dimensional Lamé system and applications to an inverse problem, in Control Theory of Partial Differential Equations, Lect. Notes Pure. Appl. Math. 242 (2005) 337-374.

[11] O.Y. Imanuvilov and M. Yamamoto, Carleman estimates for the Lamé system with stress boundary conditions and the application to an inverse problem. Publications of the Research Institute for Mathematical Sciences Kyoto University 43 (2007) 1023-1093.

[12] V. Komornik, A new method of exact controllability in short time and applications. Ann. Fac. Sci. Toulouse Math. 10 (1989) 415-464.

[13] J. Lagnese, Boundary Stabilization of Thin Plates, SIAM Studies in Applied Mathematics 10. Society for Industrial and Applied Mathematics (1989).

[14] J.E. Lagnese and J.-L Lions, Modelling, Analysis and Control of Thin Plates, Recherches en Mathématiques Appliquées RMA 6. Springer-Verlag (1989).

[15] I. Lasiecka and R. Triggiani, Exact controllability and uniform stabilization of Kirchoff plates with boundary controls only on $\left.\Delta w\right|_{\Sigma}$. J. Differ. Eqn. 93 (1991) 62-101.

[16] I. Lasiecka and R. Triggiani, Sharp regularity for elastic and thermoelastic Kirchoff equations with free boundary conditions. Rocky Mountain J. Math. 30 (2000) 981-1024.

[17] J.L. Lions, Optimal Control of Systems Governed by Partial Differential Equations. Springer-Verlag (1971).

[18] J.L. Lions, Exact controllability, stabilization and perturbations for distributed systems. SIAM Rev. 30 (1988) 1-68.

[19] D.J. Mead and S. Markus, The forced vibration of a three-layer, damped sandwich beam with arbitrary boundary conditions. J. Sound Vibr. 10 (1969) 163-175.

[20] Y.V.K.S. Rao and B.C. Nakra, Vibrations of unsymmetrical sandwich beams and plates with viscoelastic cores. J. Sound Vibr. 34 (1974) 309-326.

[21] R. Rajaram, Exact boundary controllability results for a Rao-Nakra sandwich beam. Systems Control Lett. 56 (2007) $558-567$. 
[22] R. Rajaram and S.W. Hansen, Null-controllability of a damped Mead-Markus sandwich beam. Discrete Contin. Dynam. Syst. Suppl. (2005) 746-755.

[23] C.T. Sun and Y.P. Lu, Vibration Damping of Structural Elements. Prentice Hall (1995).

[24] D. Tataru, Carleman estimates and unique continuation for solutions to boundary value problems. J. Math. Pures. Appl. 75 (1996) 367-408.

[25] X. Zhang, Explicit observability inequalities for the wave equation with lower order terms by means of Carleman inequalities. SIAM J. Control Optim. 39 (2000) 812-834. 